\title{
ON THE RECONSTRUCTION OF TOPOLOGICAL SPACES FROM THEIR GROUPS OF HOMEOMORPHISMS
}

\author{
MATATYAHU RUBIN
}

\begin{abstract}
For various classes $K$ of topological spaces we prove that if $X_{1}$, $X_{2} \in K$ and $X_{1}, X_{2}$ have isomorphic homeomorphism groups, then $X_{1}$ and $X_{2}$ are homeomorphic. Let $G$ denote a subgroup of the group of homeomorphisms $H(X)$ of a topological space $X$. A class $K$ of $\langle X, G\rangle$ 's is faithful if for every $\left\langle X_{1}, G_{1}\right\rangle,\left\langle X_{2}, G_{2}\right\rangle \in K$, if $\varphi: G_{1} \rightarrow G_{2}$ is a group isomorphism, then there is a homeomorphism $h$ between $X_{1}$ and $X_{2}$ such that for every $g \in G_{1}$ $\varphi(g)=h g h^{-1}$. Theorem 1: The following class is faithful: $\{\langle X, H(X)\rangle \mid(X$ is a locally finite-dimensional polyhedron in the metric or coherent topology or $X$ is a Euclidean manifold with boundary) and for every $x \in X \quad x$ is an accumulation point of $\{g(x) \mid g \in H(X)\}\} \cup\{\langle X, G\rangle \mid X$ is a differentiable or a $P L$-manifold and $G$ contains the group of differentiable or piecewise linear homeomorphisms $\} \cup\{\langle X, H(X)\rangle \mid X$ is a manifold over a normed vector space over an ordered field $\}$. This answers a question of Whittaker [W], who asked about the faithfulness of the class of Banach manifolds. Theorem 2: The following class is faithful: $\{\langle X, G\rangle \mid X$ is a locally compact Hausdorff space and for every open $T \subseteq X$ and $x \in T \quad\{g(x) \mid g \in H(X)$ and $g \mid(X-T)=\mathrm{Id}\}$ is somewhere dense $\}$. Note that this class includes Euclidean manifolds as well as products of compact connected Euclidean manifolds. Theorem 3: The following class is faithful: $\{\langle X, H(X)\rangle \mid$ (1) $X$ is a 0 -dimensional Hausdorff space; (2) for every $x \in X$ there is a regular open set whose boundary is $\{x\} ;$ (3) for every $x \in X$ there are $g_{1}, g_{2} \in G$ such that $x \neq g_{1}(x) \neq g_{2}(x) \neq x$, and (4) for every nonempty open $V \subseteq X$ there is $g \in H(X)-\{$ Id $\}$ such that $g \nmid(X-V)=I d\}$. Note that (2) is satisfied by 0 -dimensional first countable spaces, by order topologies of linear orderings, and by normed vector spaces over fields different from $\mathbf{R}$. Theorem 4: We prove (Theorem 2.23.1) that for an appropriate class $K^{T}$ of trees $\left\{\langle\operatorname{Aut}(T), T ; \leq, \circ, \mathrm{Op}\rangle \mid T \in K^{T}\right\}$ is first-order interpretable in $\left\{\operatorname{Aut}(T) \mid T \in K^{T}\right\}$.
\end{abstract}

\section{INTRODUCTION}

This work is concerned basically with the following question: given two topological spaces $X_{1}, X_{2}$ with isomorphic groups of autohomeomorphisms, does

Received by the editors October 12, 1987.

1980 Mathematics Subject Classification (1985 Revision). Primary 54H99; Secondary 20F38.

This work contains results that were found when the author was a doctoral student of Professor S. Shelah in the Hebrew University, Jerusalem, in 1976; during his postdoctoral period in the University of Colorado in Boulder, 1976-1978 (at that time it was supported by an NSF grant); in Ben Gurion University of the Negev, Israel, 1978-1982; and in the University of Wisconsin, Madison, 1982-1983. The final version of this work was typed in Simon Fraser University, Burnaby, British Columbia, in 1987. I thank all these institutions and the National Science foundation for supporting me. 
it follow that $X_{1}$ and $X_{2}$ are homeomorphic? In general the answer to this question is negative. It is well known (see, e.g., [Rr] or [S]) that there are many nonhomeomorphic compact Hausdorff spaces with a trivial group of autohomeomorphisms. But also among topological spaces that have many homeomorphisms one can easily find nonhomeomorphic spaces that have the same homeomorphism group; take, e.g., $[0,1]$ and $(0,1)$. However, for some wide and natural classes $K$ of topological spaces it is true that if two spaces in $K$ have isomorphic groups of homeomorphisms, then the spaces are homeomorphic.

The method of proof yields results which are stronger in three directions. (a) It is the case not only that spaces which have isomorphic groups of homeomorphisms are homeomorphic but also that every isomorphism between $H(X)$ and $H(Y)$ is induced by a homeomorphism between $X$ and $Y$. This means that if $X$ belongs to a faithful class, then every automorphism of $H(X)$ is inner.

(b) In order to conclude that $X$ and $Y$ are homeomorphic, we do not have to assume that $H(X)$ and $H(Y)$ are isomorphic; in fact, it suffices to assume that some rich enough subgroups of $H(X)$ and $H(Y)$ are isomorphic. This strengthening has many natural applications, e.g., groups of differentiable and Lipschitz homeomorphisms of differentiable manifolds, groups of measurepreserving automorphisms of measure algebras, and groups of automorphisms of certain linear orderings.

(c) It might be of interest to model theorists that in many cases dealt with in this paper we obtain a first-order interpretation of $\left\langle X, \tau_{X}, H(X) ; \in, \mathrm{Op}\right\rangle$ in $H(X)$. This fact gives rise to the following theorem, which will appear in [R3].

Theorem 0.1. (Assume Gödel's axiom of constructibility.) If $X$ and $Y$ are second countable connected Euclidean manifolds and $H(X)$ is elementarily equivalent to $H(Y)$, then $X$ and $Y$ are homeomorphic.

Description and discussion of the results. Let $R(X)$ denote the Boolean algebra of regular open sets of a topological space $X$. For a subgroup $G$ of $H(X)$ let $H R(X, G)$ be the following structure: $\langle G, R(X) ; \subseteq, \circ, \mathrm{Op}\rangle ; \subseteq$ denotes the inclusion relation on $R(X)$, $\circ$ is the composition operation on $G$, and $\mathrm{Op}=\{\langle f, U, V\rangle \mid f \in G, U, V \in R(X)$, and $f(U)=V\}$.

In $\S 2$, Theorem $2.14(\mathrm{a})$, we prove the most fundamental result in this work. We show that under appropriate assumptions $(*)$ on $X$ and $G$ one can reconstruct $H R(X, G)$ from $G$. The direct outcome of this reconstructibility result is the following theorem. For $g \in H(X)$ let $g^{\dagger}$ denote the automorphism that $g$ induces on $R(X)$.

Theorem 0.2. Suppose $\left\langle X_{1}, G_{2}\right\rangle$ and $\left\langle X_{2}, G_{2}\right\rangle$ satisfy $(*)$, and let $\varphi: G_{1} \rightarrow$ $G_{2}$ be an isomorphism between $G_{1}$ and $G_{2}$; then there is an isomorphism $\tau$ between $\operatorname{HR}\left(X_{1}, G_{1}\right)$ and $H R\left(X_{2}, G_{2}\right)$ which induces $\varphi$, that is, for every $g \in$ $G_{1} \varphi(g)^{\dagger}=\tau \circ g^{\dagger} \circ \tau^{-1}$.

Let us explain the most useful (but not the most general) version of $(*)$. Let $G \subseteq H(X)$; we say that $\langle X, G\rangle$ is regionally disrigid if for every nonempty 
open $V \subseteq X$ there is $g \in G-\{$ Id $\}$ such that $g \uparrow(X-V)=$ Id. We say that $X$ is regionally disrigid when $\langle X, H(X)\rangle$ is. Note that regional disrigidity implies that $X$ has no isolated points. However, our results can easily be extended to spaces with isolated points. Let $G \subseteq H(X)$ and $U \subseteq X$ be open; $U$ is flexible with respect to $G$ if for every open $V, W \subseteq U$ : if there is $g \in G$ such that $g(V) \cap W \neq \varnothing$, then there is $g \in G$ such that $g \uparrow(X-U)=$ Id and $g(V) \cap W \neq \varnothing$.

(*) means that (1) $X$ is a Hausdorff space, (2) $\langle X, G\rangle$ is regionally disrigid, and (3) for every nonempty open $U \subseteq X$ there is a nonempty open $U_{1} \subseteq U$ such that $U_{1}$ is flexible with respect to $G$.

(*) has two advantages: first it is satisfied by numerous natural classes of $\langle X, G\rangle$ 's; second, the fact that a certain $\langle X, G\rangle$ satisfies $(*)$ is usually a trivial fact.

Even though Theorem 2.14(a) is formulated in topological terms it is indeed a purely Boolean algebraic result. Here is maybe a more transparent formulation of that theorem. Let $B$ be a complete Boolean algebra (BA) and $G$ be a subgroup of $\operatorname{Aut}(B)$. We say that $\langle B, G\rangle$ is regionally disrigid if for every $a \in B-\{0\}$ there is $g \in G-\{$ Id $\}$ such that $g(b)=b$ for every $b$ which is disjoint from $a$. We say that $a \in B$ is flexible in $\langle B, G\rangle$ if, for every $c, d \leq a$, if there is $g \in G$ such that $g(c) \cap d \neq 0$ then there is $f \in G$ such that $f(c) \cap d \neq 0$ and $f(b)=b$ for every $b$ which is disjoint from $a$. We say that $\langle B, G\rangle$ is flexible if $\{a \in B \mid a$ is flexible in $\langle B, G\rangle\}$ is dense in $B$.

Let $M(B, G)=\langle B, G ; \leq, \circ, \mathrm{Op}\rangle, K_{1}=\{M(B, G) \mid\langle B, G\rangle$ is regionally disrigid and flexible $\}$, and $K_{0}=\left\{G \mid \exists B\left(M(B, G) \in K_{1}\right)\right\}$. The following is a reformulation of Theorem 0.2 .

Theorem $0.2^{*}$. $K_{1}$ is first-order interpretable in $K_{0}$.

In addition to the classes mentioned in the abstract the following classes satisfy $(*)$ :

$\{\langle X, H(X)\rangle \mid X$ is a locally convex vector space over an ordered field $\}$,

$\{\langle X, H(X)\rangle \mid X$ is a product of Euclidean manifolds $\}$,

$\{\langle X, H(X)\rangle \mid X$ is 0 -dimensional and regionally disrigid $\}$; for an infinite cardinal $\kappa\left\{\langle X, H(X)\rangle \mid X=\prod_{i \in I}^{\kappa} X_{i}, X_{i}\right.$ is a Euclidean manifold $\}, \prod_{i \in I}^{\kappa} X_{i}$ means the $\kappa$-box product of the $X_{i}$ 's; that is, a basic open set has the form $\prod_{i \in I} U_{i}$ where $U_{i}$ is open in $X_{i}$ and $\left|\left\{i \mid U_{i} \neq X_{i}\right\}\right|<\kappa$. More classes satisfying $(*)$ are mentioned in 2.23.2.

It is, however, not clear whether Theorem 0.2 is as general as it should be. We do. not know the answer to the following question.

Question 0.1. Does Theorem 0.2 remain true if $(*)$ is replaced by the requirement that $\left\langle X_{i}, G_{i}\right\rangle$ be Hausdorff and regionally disrigid? We do not know the answer to this question even when the $G_{i}$ 's are taken to be $H\left(X_{i}\right)$.

In fact, there are some pathological cases in which $\langle X, G\rangle$ and even $\langle X$, $H(X)\rangle$ are regionally disrigid but they do not satisfy $(*)$. 
Example 0.3. Let $X_{0}$ be the torus which is obtained by identifying each two opposite sides of the unit square. If $\alpha$ is an irrational number and $\mathscr{L}$ is the family of all lines in the plane whose slope is $\alpha$, then $\mathscr{L}$ induces a foliation $\mathscr{F}$ of $X_{0}$. Let $G_{0}$ be the group of homeomorphisms of $X_{0}$ which leave each leaf of $\mathscr{F}$ invariant. Clearly $\left\langle X_{0}, G_{0}\right\rangle$ is regionally disrigid. It is easy to see that $\left\langle X_{0}, G_{0}\right\rangle$ does not satisfy $(*)$. If $X_{1}$ is a subspace of $X_{0}$ consisting of one leaf of the above foliation then $\left\langle X_{1}, H\left(X_{1}\right)\right\rangle$ does not satisfy $(*)$. If $X_{2}=X_{1}^{\aleph_{0}}$, then for every open $U \subseteq X_{2}$ and for every $G \subseteq H(U) G$ does not satisfy (*).

According to Ling [ $\mathrm{Lg} 1]$, the smooth version of $\left\langle X_{0}, G_{0}\right\rangle$ belongs to a faithful class. However, his method does not cover $X_{1}$ or $X_{2}$.

The reader should realize that groups of analytic homeomorphisms or linear isomorphisms do not fall into the framework of this work because such homeomorphisms are never the identity outside a regular open proper subset of $X$.

Theorem 0.2 is just an intermediate step in proving faithfulness results. In $\S 3$ we reconstruct $\left\langle X, \tau_{X}\right\rangle$ from $H R(X, G)$, thus obtaining the final results mentioned in the abstract.

Unfortunately, we do not have one naturally defined faithful class which contains all the concrete classes for which we can prove faithfulness. $\S 3$ is thus divided into parts, each of them dealing with another faithful class. In $\S 3.1$, Corollary $3.13(\mathrm{c})$, we prove that the class $K^{L C} \stackrel{\text { def }}{=}\{\langle X, G\rangle \mid X$ is a locally compact Hausdorff space and for every open $U$ and $x \in U \quad\{g(x) \mid g \in G$ and $g\{(X-U)=\mathrm{Id}\}$ is somewhere dense $\}$ is faithful.

One might ask whether some of the conditions here can be removed or weakened. The local compactness cannot be discarded. Van Mill [Ml] constructed two nonhomeomorphic subsets of $S^{2}$ which are very homogeneous and have the same group of homeomorphisms.

In §3.II and §3.III we define and prove the faithfulness of a class $K^{M}$ of $\langle X, G\rangle$ 's, and in $\S 3 . I V$ we show that $K^{M}$ contains three main classical subclasses: (1) Euclidean manifolds with boundary, (2) polyhedra that do not contain an infinite increasing chain of simplexes, and (3) manifolds over locally convex linearly bounded topological vector spaces over an ordered field. A topological vector space is called linearly bounded if it contains a nonempty open set $U$ such that for every straight line $l, U \cap l$ is bounded in $l$.

For polyhedra and manifolds with boundary we have an additional requirement $(* *)$, namely that for every $x \in X \quad\{g(x) \mid g \in H(X)\}$ does not have isolated points. Clearly such a requirement is necessary in order to exclude one of the spaces $(0,1)$ or $[0,1]$, which have the same group of homeomorphisms. But even when considering just compact polyhedra, $(* *)$ has to be required. Take, e.g., the following spaces shown in Figure 1, where each segment in the sketch denotes a copy of $[0,1]$ and the circle denotes a copy of the 1-dimensional sphere. Certainly $H\left(X_{1}\right) \cong H\left(X_{2}\right)$ but $X_{1}$ and $X_{2}$ are not homeomorphic. 

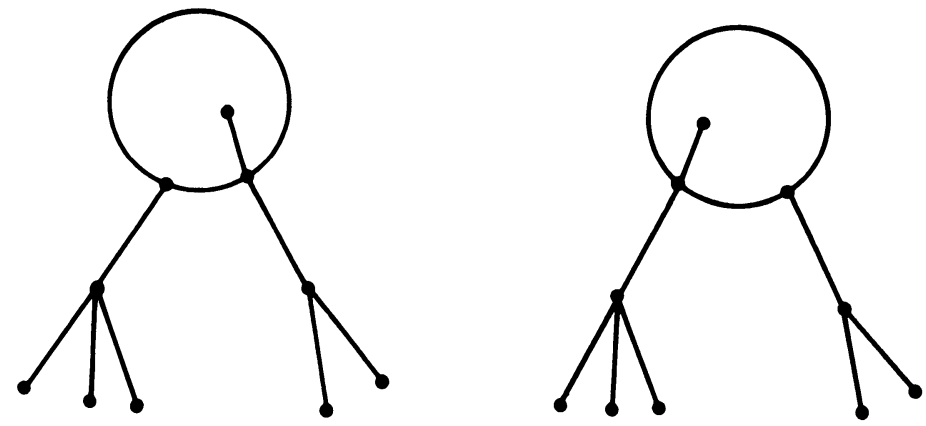

FIGURE 1

In $\S 3 . \mathrm{V}$ we prove that the class $K^{T D}$ defined below is faithful. $K^{T D}=$ $\{\langle X, G\rangle \mid$ (1) $X$ is 0-dimensional and Hausdorff; (2) $\langle X, G\rangle$ is regionally disrigid; (3) for every $x \in X$ there are $g_{1}, g_{2} \in G$ such that $x \neq g_{1}(x) \neq$ $g_{2}(x) \neq x$; (4) for every $x \in X$ there is a regular open set $V$ such that $\{x\}=\operatorname{bd}(V)$; and (5) for every clopen set $V \subseteq X$ and $g \in G$ : if $g(V)=V$ then $g \nmid V \cup \operatorname{Id} \uparrow(X-V) \in G$, and if $g(V) \cap V=\varnothing$, then $g\left|V \cap g^{-1}\right|$ $g(V) \cup \operatorname{Id} \uparrow(X-V-g(V)) \in G\}$.

Note that (5) is automatically satisfied by $H(X)$. Condition (4) seems to be quite restrictive; however, it is impossible to remove it without introducing some other condition instead. Van Douwen constructed two very homogeneous subsets of $\{0,1\}^{\aleph_{1}} X_{1}, X_{2}$ such that $X_{1}$ and $X_{2}$ are nonhomeomorphic, $H\left(X_{1}\right) \cong H\left(X_{2}\right)$, and they satisfy (1), (2), and (3) in the definition of $K^{T D}$.

We were unsuccessful in proving the faithfulness of the following classes.

Question 0.2. Are the following classes faithful?

(a) $K^{P M}=\left\{\langle X, H(X)\rangle \mid X=\prod_{i \in I} X_{i}\right.$ for each $i \in I, X_{i}$ is a Euclidean manifold\}.

See (5) and (6) in the list of faithful classes. R \}.

(b) $K^{L C X}=\{\langle X, H(X)\rangle \mid X$ is a locally convex topological vector space over

See (7) in the list of faithful classes.

(c) $\{\langle X, H(X)\rangle \mid X$ is a box product of Euclidean manifolds $\}$.

In $\S 3$.VI we prove some faithfulness results that were found during the revision of this paper. It includes proofs of the faithfulness of the following classes: (1) manifolds over locally convex linearly bounded vector spaces over ordered fields, (2) $\mathbf{R} \times \mathbf{Q}$ and similar spaces, and (3) products of countably many compact Euclidean manifolds.

A list of concrete faithful classes. The following classes are faithful.

(1) $\{\langle X, H(X)\rangle \mid X$ is a manifold with a boundary and for every $x \in X$ $\{g(x) \mid g \in H(X)\}$ has no isolated points $\}$. This class is contained in $K^{M}$; see 3.43 for the proof. 
(2) $\{\langle X, H(X)\rangle \mid X$ is a polyhedron with either the coherent or the metric topology and for every $x \in X \quad\{g(x) \mid g \in H(X)\}$ has no isolated points $\}$. This class is contained in $K^{M}$; see 4.34 for the proof.

(3) $\{\langle X, G\rangle \mid X$ is a manifold with a $P L$-structure and $P L(X) \subseteq G \subseteq H(X)\}$ where $P L(X)$ is the group of $P L$-homeomorphisms. This class is contained in both $K^{L C}$ and $K^{M}$.

(4) For $0<k \leq \infty\left\{\langle X, G\rangle \mid X\right.$ is a $C^{k}$-manifold, $\left.C^{k}(X) \subseteq G \subseteq H(X)\right\}$, where $C^{k}(X)$ is the group of $C^{k}$-homeomorphisms. This is a subclass of both $K^{L C}$ and $K^{M}$.

There are numerous variants of (4) which are faithful: Lipschitz homeomorphism of a manifold with a Lipschitz atlas, quasi-conformal homeomorphisms of a smooth manifold with a quasi-conformal atlas, volume-preserving homeomorphisms, etc.

(5) $\left\{\langle X, H(X)\rangle \mid X=X^{\prime} \times \prod_{i \in I} X_{i}, X^{\prime}\right.$ is a Euclidean manifold and for every $i \in I, X_{i}$ is a compact connected Euclidean manifold $\}$. This class is contained in $K^{L C}$.

(6) $\left\{\langle X, H(X)\rangle\left|X=\prod_{i \in I} X_{i},\right| I \mid=\aleph_{0}\right.$ for each $i \in I, X_{i}$ is a compact manifold\}. This class is dealt with in $\S 3$.VI.

(7) $\left\{\langle X, H(X)\rangle \mid\right.$ for some infinite cardinal $\lambda, X$ is a manifold over $\left.[0,1]^{\lambda}\right\}$. This class is contained in $K^{L C}$.

(8) $\{\langle X, H(X)\rangle \mid X$ is a manifold over a locally convex topological vector space $Y$ over an ordered field $F$, and $Y$ has an open set $U$ which intersects each line in a bounded set and if $\mathbf{R}$ is not embeddable in $F$ then the above $U$ is required to be clopen\}. This class is contained in $K^{M}$ as redefined in $\S 3$.VI.

Note that at least for $F=\mathbf{R}$ the last clause in (8) is equivalent to the fact that $Y$ admits a continuous norm. Unfortunately, (8) does not include weak topologies on Banach spaces.

We can also include $\langle Y, H(Y)\rangle$ where $Y$ is the complement of a normed vector space over $\mathbf{R}$ in its completion. The same proof as for (8) works.

(9) $\{\langle X, G\rangle \mid X$ is a Banach space and $L(X) \subseteq G \subseteq H(X)$ where $L(X)$ is the group of Lipschitz homeomorphisms of $X\}$. Note that in particular this class includes the group of uniformly continuous homeomorphisms of a Banach space. $X$ can be replaced by a Banach manifold with a Lipschitz atlas. The proof that the above class is faithful will appear in [RY].

(10) $\{\langle B, \mu\rangle, \operatorname{Aut}(\langle B, \mu\rangle) \mid\langle B, \mu\rangle$ is an atomless $\sigma$-finite measure algebra $\}$. Note that the automorphism group of $\langle B, \mu\rangle$ can be regarded as a group of homeomorphisms of the Stone space of $B$. This is a subclass of $K^{L C}$.

(11) $\{\langle\langle L,<\rangle, \operatorname{Aut}(\langle L,\langle\rangle)\rangle|\langle L,<\rangle$ is a Dedekind complete linear ordering which has a dense 2-transitive suborder $\}$. This is a subclass of $K^{L C}$.

(12) $\{\langle B, \operatorname{Aut}(B)\rangle \mid B$ is a complete Boolean algebra which does not have rigid factors $\}$. This follows from $\S 2$ because if $B$ is a complete Boolean algebra, then $B$ is isomorphic to $R(S(B))$ where $S(B)$ is the Stone space of $B$. 
(13) A tree is a partially ordered set $\langle T,<\rangle$ such that for every $x \in T$ $\{y \mid y<x\}$ is linearly ordered by $<$. A subset $b \subset T$ is a branch if it is a maximal subset of $T$ which is linearly ordered by $<$. Let $B(T)$ denote the set of branches of $T$. For every $x \in T$ let $U_{x}=\{b \in B(T) \mid x \in b\}$. Let $\tau_{T}$ be the following topology on $B(T)$ : a base for $\tau_{T}$ is $\left\{U_{x} \mid x \in T\right\}$. We regard $B(T)$ as a topological space with the topology $\tau_{T}$. T is homogeneous if (1) for every $x \in T$ there is $f \in \operatorname{Aut}(\langle T,<\rangle)$ such that $f(x) \neq x$ and (2) for every $x \in T$ there is $f \in \operatorname{Aut}(T)$ such that $f(x)=x$ and for some $y>x \quad f(y) \neq y .\{\langle B(T), H(B(T))\rangle \mid T$ is a homogeneous tree $\}$. This class is contained in $K^{T D}$.

(14) $\{\langle X, H(X)\rangle \mid X$ has the order topology of a 0-dimensional linear ordering $\langle L,<\rangle$ and for every $a<b$ in $L$ there is $f \in \operatorname{Aut}(\langle L,<\rangle)$ such that $f \uparrow(a, b) \neq \operatorname{Id}$ and $f((a, b)) \cap(a, b) \neq \varnothing$; and for every $a \in L$ there is $f \in \operatorname{Aut}(\langle L,<\rangle)$ such that $f(a) \neq a\}$.

(15) $\{\langle X, G\rangle \mid X$ is 0-dimensional and first countable, for every $x \in X$ $|\{g(x) \mid g \in G\}| \geq 3$ and $\langle X, G\rangle$ is regionally disrigid $\}$. This class is contained in $K^{T D}$.

(16) $\{\langle X, H(X)\rangle \mid X$ is a box product of members from (13), (14), and (15)\}. This class is contained in $K^{T D}$.

(17) $\left\{\langle X, H(X)\rangle \mid X=Y \times Z\right.$ where $Y \in K^{M}$ and $\left.Z \in K^{T D}\right\}$. Let $\mathbf{Q}$, I, $\mathbf{C}$ denote respectively the rationals, the irrationals, and the Cantor set; then $\mathbf{R} \times \mathbf{Q}, \mathbf{R} \times \mathbf{I}$, and $\mathbf{R} \times \mathbf{C}$ belong to the above class. The above class is dealt with in $\S 3$. VI.

Some classes in the above list call for stronger results. If $X$ carries some structure $S$ in addition to its topology, and we consider the group of homeomorphisms which preserve $S$, we would like to know that an isomorphism between such groups is induced by a homeomorphism that preserves $S$.

Such questions arise in (3), (4), (9), (10), and (11).

The fact that an isomorphism between $C^{k}(X)$ and $C^{k}(Y)$ is induced by a $C^{k}$-homeomorphism and the analogous fact for $P L(X)$ were proved by Ling in [Lg1]. Many other structures on a smooth manifold are considered there. In [RY] we will give an alternative proof of the smooth case and will consider variants of that question. The case of the groups of Lipschitz and uniformly continuous homeomorphisms of a Banach manifold will be dealt with in [RY].

The case of 2-transitive linear orderings was dealt with in [GGHJ]. It follows easily from $\S 2$.

There is another type of question that we did not deal with. There are cases in which $H(X)$ is embeddable in $H(Y)$. Are there any reasonable assumptions on the type of the embeddings so that the embeddability of $H(X)$ in $H(Y)$ will imply that $X$ is some kind of continuous image of $Y$ ?

Some historical remarks. The faithfulness of the class of Euclidean manifolds was proved by Whittaker in [W]. McCoy [M] extended Whittaker's results to Hilbert cube manifolds. Independently of our work, Ling [Lg1] proved the 
faithfulness of the smooth and the $P L$ cases and numerous variants of these cases. Ling's work also covers Euclidean manifolds and proves that isomorphism between the groups of $S$-preserving homeomorphisms is induced by an $S$-preserving homeomorphism ( $S$ is some structure on a manifold).

The method of Whittaker as well as the method of Ling is entirely diffrent from ours. Both methods seem to require that $X$ will be locally compact. Whittaker's method seems to require that $G$ will be closed under some infinitary composition. Ling assumes that $G$ or at least some nontrivial normal subgroup of $G$ is factorizable (see [Lg1]). This excludes 0 -dimensional spaces. On the other hand, Ling's work includes some classes which are not included in ours, e.g., manifolds with smooth foliations.

Later, Filipkiewicz [Fl], not knowing about Ling's results, reproved the differentiable case.

\section{Preliminaries}

Most of the notions will be defined when they are first used. The terminology of model theory is very convenient for this work. Nevertheless, no theorem of model theory will be needed.

$\omega$ denotes the set of natural numbers; $|A|$ denotes the cardinality of the set A.

We use the terminology of [CK] for languages, structures and satisfaction. See [CK, pp. 18-33], but 1.3.4-1.3.11 is not needed here. "A model" and "a structure" mean the same thing. We denote a structure usually, but not always, by the letters $M, N .|M|$ denotes the universe of $M$.

We make an abuse of notation and denote individual variables and elements of the model by the same letters; so $\varphi(f, g)$ means that $\varphi$ is a formula all of whose free variables are among $\{f, g\}$, whereas $M \vDash \varphi[f, g]$ means that $\varphi$ is a formula with at most two free variables and the pair $\langle f, g\rangle$ satisfies $\varphi$ in $M$.

A group $G$ is considered as the structure $\left\langle G, \cdot,^{-1}\right.$, Id $\rangle$; that is, it is a structure in a language which contains one binary function symbol, one unary function symbol, and one individual constant. A topological space $\left\langle X, \tau_{X}\right\rangle$ is regarded as the following structure: $\left\langle X \cup \tau_{X}, P, Q, \in\right\rangle$ where $P$ is a unary predicate and $P=X, Q$ is a unary predicate and $Q=\tau_{X}$, and $\in$ is the belonging relation between elements of $X$ and elements of $\tau_{X}$.

$M \cong N$ means that $M$ and $N$ are isomorphic, in particular $M$ and $N$ have to be in the same language. $X \cong Y$ means that the topological spaces $X$ and $Y$ are homeomorphic.

Let $M_{1}, \ldots, M_{l}$ be structures in disjoint languages and $R_{1}, \ldots, R_{l}$ be relations on $\bigcup_{i=1}^{k}\left|M_{i}\right|$. (Note that an $n$-place function can be regarded as an $n+1$-place relation.) $\left\langle M_{1}, \ldots, M_{k} ; R_{1}, \ldots, R_{l}\right\rangle$ denotes the structure with universe $\bigcup_{i=1}^{k}\left|M_{i}\right|$ whose relations and functions are those of $M_{1}, \ldots, M_{k}$, 
the relations $R_{1}, \ldots, R_{l}$, and in addition for every $i \leq k$ a unary relation $P_{i}$ to denote $\left|M_{i}\right|$.

$\left\langle M_{1}, \ldots, M_{k} ;\right\rangle$ is of course a special case of the above notation. If $M_{1}, \ldots$, $M_{r}$ are structures, $A_{r+1}, \ldots, A_{k}$ are sets, and $R_{1}, \ldots, R_{l}$ are relations on $\bigcup_{i=1}^{r}\left|M_{i}\right| \cup \bigcup_{i=r+1}^{k} A_{i}$, then $\left\langle M_{1}, \ldots, M_{r}, A_{r+1}, \ldots, A_{k} ; R_{1}, \ldots, R_{l}\right\rangle$ denotes the structure $\left\langle M_{1}, \ldots, M_{k} ; R_{1}, \ldots, R_{l}\right\rangle$, where for every $r<i \leq k, M_{i}$ is the structure without relations or functions and with universe $A_{i}$.

We say that $M$ and $N$ are elementarily equivalent $(M \equiv N)$ if they have the same language $L$ and for every sentence $\varphi \in L \quad M \vDash \varphi$ iff $N \vDash \varphi$.

Interpretations.

Definition 1.1. Let $K$ be a class of models in the language $L, K^{*}$ be a class of models in the language $L^{*}$, and $R \subseteq K \times K^{*}$ be a relation. We say that $K^{*}$ is first-order interpretable in $K$ relative to $R$ if there are formulas in $L$ $\varphi_{U}\left(x_{1}, \ldots, x_{n}\right), \varphi_{\mathrm{Eq}}\left(x_{1}, \ldots, x_{n}, y_{1}, \ldots, y_{n}\right)$, for every $m$-place relation symbol $P \in L^{*} \varphi_{P}\left(x_{1}^{1}, \ldots, x_{n}^{1}, \ldots, x_{1}^{m}, \ldots, x_{n}^{m}\right)$, and for every $m$-place function symbol $F \in L^{*} \varphi\left(x_{1}^{1}, \ldots, x_{n}^{1}, \ldots, x_{1}^{m}, \ldots, x_{n}^{m}, y_{1}, \ldots, y_{n}\right)$ such that, for every $M \in K$ and $M^{*} \in K^{*}$, if $\left\langle M, M^{*}\right\rangle \in R$, then there is a function $h:\left\{\left\langle a_{1}, \ldots, a_{n}\right\rangle \mid M \vDash \varphi_{U}\left[a_{1}, \ldots, a_{n}\right]\right\} \stackrel{\text { onto }}{\rightarrow}\left|M^{*}\right|$ such that $(1)$ for every $\left\langle a_{1}, \ldots, a_{n}\right\rangle,\left\langle b_{1}, \ldots, b_{n}\right\rangle \in \operatorname{Dom}(h) h\left(\left\langle a_{1}, \ldots, a_{n}\right\rangle\right)=h\left(\left\langle b_{1}, \ldots, b_{n}\right\rangle\right)$ iff $M \vDash \varphi_{\mathrm{Eq}}\left[a_{1}, \ldots, a_{n}, b_{1}, \ldots, b_{n}\right] ;(2)$ for every $m$-place relation symbol $P \in L^{*}$ and $\left\langle a_{1}^{i}, \ldots, a_{n}^{i}\right\rangle \in \operatorname{Dom}(h), i=1, \ldots, m: M \vDash \varphi_{P}\left[a_{1}^{1}, \ldots, a_{n}^{1}, \ldots\right.$, $\left.a_{1}^{m}, \ldots, a_{n}^{m}\right]$ iff $\left\langle h\left(\left\langle a_{1}^{1}, \ldots, a_{n}^{1}\right\rangle\right), \ldots, h\left(\left\langle a_{1}^{m}, \ldots, a_{n}^{m}\right\rangle\right)\right\rangle \in P^{M^{*}} ;$ and (3) for every $m$-place function symbol $F \in L^{*},\left\langle a_{1}^{i}, \ldots, a_{n}^{i}\right\rangle \in \operatorname{Dom}(h), i=1, \ldots$, $m$, and

$$
\left\langle b_{1}, \ldots, b_{n}\right\rangle \in \operatorname{Dom}(h): M \vDash \varphi_{F}\left[a_{1}^{1}, \ldots, a_{n}^{1}, \ldots, a_{1}^{m}, \ldots, a_{n}^{m}, b_{1}, \ldots, b_{n}\right]
$$

iff $F^{M^{*}}\left(h\left(\left\langle a_{1}^{1}, \ldots, a_{n}^{1}\right\rangle\right), \ldots, h\left(\left\langle a_{1}^{m}, \ldots, a_{n}^{m}\right\rangle\right)\right)=h\left(\left\langle b_{1}, \ldots, b_{n}\right\rangle\right)$.

Proposition 1.2. Let $K^{*}$ be first-order interpretable in $K$ relative to $R$.

(a) If $M_{1}, M_{2} \in K, M_{1}^{*}, M_{2}^{*} \in K^{*},\left\langle M_{i}, M_{i}^{*}\right\rangle \in R, i=1,2$, and $M_{1} \cong M_{2}$, then $M_{1}^{*} \cong M_{2}^{*}$.

(b) If in (a) $\cong$ is replaced by $\equiv$, then the claim obtained in this way is true.

Proof. Easy.

If $K^{*}$ is first-order interpretable in $K$ relative to $R$ and $\left\langle M, M^{*}\right\rangle \in R$, then we represent the elements of $\left|M^{*}\right|$ by elements of $|M|^{n}$; there are cases in which $M^{*}$ can be reconstructed from $M$ but its elements are represented by objects more complicated than $n$-tuples from $M$. Typical such cases are Stone spaces and Boolean algebras; the elements of the Stone space of a Boolean algebra $B$ are represented by ultrafilters which are elements of the power set of $|M|$ rather than elements of $|M|^{n}$. Our next goal is thus to define a notion of interpretation more general than first-order interpretation. Let $P(A)$ denote 
the power set of $A$. If $M$ is a model, we define the second-order model based on $M$, and it will be denoted by $M^{[2]}$.

$$
\begin{gathered}
M^{[2]}=\left\langle M, P(|M|), P\left(|M|^{2}\right), \ldots ; \varepsilon_{1}, \varepsilon_{2}, \ldots\right\rangle \quad \text { where } \varepsilon_{i} \subseteq|M|^{i} \times P\left(|M|^{i}\right), \\
\left\langle a_{1}, \ldots, a_{i}, r\right\rangle \in \varepsilon_{i} \quad \text { iff }\left\langle a_{1}, \ldots, a_{i}\right\rangle \in r .
\end{gathered}
$$

Remark. It turns out that all relations on a set $A$ can be encoded by binary relations on $A$; so as far as we are concerned $M^{[2]}$ can be replaced by $\left\langle M, P\left(|M|^{2}\right) ; \varepsilon_{2}\right\rangle$.

Definition 1.3. Let $K, K^{*}, L, L^{*}$, and $R$ be as in Definition 1.1. We say that $K^{*}$ is second-order interpretable in $K$ relative to $R$ if $K^{*}$ is first-order interpretable in $\left\{M^{[2]} \mid M \in K\right\}$.

All interpretation that we shall obtain will be either first or second order, but it is possible to define more general notions of interpretation. For every model $M$ and an ordinal $\alpha$ it is possible to define the $\alpha$ 's order model based on $M$, which will be denoted by $M^{[\alpha]}$.

Definition 1.4. Let $K, K^{*}, L, L^{*}$, and $R$ be as in 1.3. We say that $K^{*}$ is interpretable in $K$ relative to $R$ if for some $\alpha, K^{*}$ is first-order interpretable in $\left\{M^{[\alpha]} \mid M \in K\right\}$.

A formula in the language of $M^{[2]}$ is called a second-order formula in the language of $M$. If $\varphi$ is a second-order formula in the language of $M$, then $M \vDash \varphi\left[a_{1}, \ldots, a_{n}, r_{1}, \ldots, r_{k}\right]$ means that $M^{[2]} \vDash \varphi\left[a_{1}, \ldots, a_{n}, r_{1}, \ldots, r_{k}\right]$.

A formula in the language of $M^{[2]}$ which of the relation symbol $\varepsilon_{1}, \ldots$, $\varepsilon_{n}, \ldots$, mentions at most $\varepsilon_{1}$ is called a monadic formula in the language of $M$. Hence, intuitively speaking, a monadic formula in the language of $M$ is a formula that speaks on elements of $|M|$ and on subsets of $|M|$ but does not speak about $n$-place relations on $|M|$ for any $n>1$.

\section{THE RECONSTRUCTION OF the Boolean Algebra of Regular OPEN SETS}

Throughout this section we shall be concerned with structures of the form $\left\langle X, \tau_{X}, G ; \in, \mathrm{Op}\right.$, ○ $\rangle$ where $\left\langle X, \tau_{X}\right\rangle$ is a topological space, $G$ is a subgroup of the group $H(X)$ of all homeomorphisms of $X, \in$ is the belonging relation between elements of $X$ and elements of $\tau_{X}$, Op $\stackrel{\text { def }}{=}\{\langle f, x, y\rangle \mid f \in G$, $x, y \in X \cup \tau_{X}$, and $f(x)=y$, and $\circ$ is the composition operation in $G$. $\left\langle X, \tau_{X}, G ; \in\right.$, Op , o $\rangle$ will be denoted by $M(X, G)$, and $M(X, H(X))$ will be abbreviated by $M(X)$.

In the sequel we shall define some formulas in the language of groups and show that they represent certain sets and relations to $M(X, G)$. Though the final interpretation in 2.17 is a first-order interpretation, it seems both convenient and more general to use, in the intermediate steps, monadic second-order formulas, i.e., formulas in which there are variables which represent subsets of G. 
We need some terminology. We assume that $X$ denotes a fixed but arbitrary topological space; $\tau_{X}$ denotes the topology of $X . G$ denotes a fixed but arbitrary subgroup of $H(X) . f, g, h$ denote elements of $G$; they are also used as variables ranging over elements of $G$. Subsets of $G$ will be denoted by bold letters $\mathbf{f}, \mathbf{g}, \mathbf{h}$. Also, $\mathbf{f}, \mathbf{g}, \mathbf{h}$ are used as variables ranging over subsets of $G$. If $\varphi(\mathbf{f}, \mathbf{g}, \ldots)$ is a monadic formula then $\varphi(f, g, \ldots)$ means $\varphi(\{f\},\{g\}, \ldots)$.

Let $A \subseteq X . \operatorname{int}(A)$ and $\operatorname{cl}(A)$ denote the interior and the closure of $A$, respectively. For subsets $A, B \subseteq X$ let $A+B \stackrel{\text { def }}{=} \operatorname{int}(\operatorname{cl}(A \cup B))$ and $\sim B=$ $\operatorname{int}(X-B)$. Let $A \sim B \stackrel{\text { def }}{=} A \cap \sim B . U \subseteq X$ is called a regular set if $U=U+U$. Clearly, a regular set is open. $R(X)$ denotes the set of regular sets of $X . U$, $V, W$ denote regular sets. It is well known that $\langle R(X),+, \cap, \sim\rangle$ is a complete Boolean algebra, its induced partial ordering is the set inclusion relation, and if $\mathscr{V} \subseteq R(X)$, then the supremum of $\mathscr{V}, \sum \mathscr{V}$, and the infimum of $\mathscr{V}, \Pi \mathscr{V}$, are $\operatorname{int}(\operatorname{cl}(\bigcup \mathscr{V}))$ and $\operatorname{int}(\bigcap \mathscr{V})$, respectively.

If $F$ is a closed set then $\operatorname{int}(F)$ is regular. So for every $A \subseteq X, A+A$ is regular. Let $\operatorname{var}(f) \stackrel{\text { def }}{=} \operatorname{int}(\operatorname{cl}(\{x \mid f(x) \neq x\}))$ and $\operatorname{fix}(f)=\sim \operatorname{var}(f)$. Clearly $\operatorname{var}(f), \operatorname{fix}(f) \in R(X)$. Let $\operatorname{var}(\mathbf{f})=\sum\{\operatorname{var}(f) \mid f \in \mathbf{f}\}$ and $\operatorname{fix}(\mathbf{f})=\sim \operatorname{var}(\mathbf{f})$. Let $Q(T) \stackrel{\text { def }}{=}\{f \mid \operatorname{var}(f) \subseteq T\}$.

We need some group theoretic notation. Id denotes the identity mapping. $f^{h} \stackrel{\text { def }}{=} h f h^{-1},[f, h] \stackrel{\text { def }}{=} f h f^{-1} h^{-1},[f, h, g] \stackrel{\text { def }}{=}[[f, h], g] . \mathbf{f}^{\mathbf{h}} \stackrel{\text { def }}{=}\left\{f^{h} \mid f \in\right.$ $\mathbf{f}, h \in \mathbf{h}\},[\mathbf{f}, \mathbf{h}], f^{\mathbf{h}},[\mathbf{f}, h]$, etc. are defined similarly.

Our policy is to add assumptions just at the first time they are needed, so we gradually add more and more assumptions on $M(X, G)$.

Assumption 2.1. $X$ is assumed to be a Hausdorff space.

Lemma 2.1. (a) Let $f_{1}, \ldots, f_{n} \in G, x \in X$ and for every $1 \leq i<j \leq n$ $f_{i}(x) \neq f_{j}(x)$, then there is an open neighborhood $T$ of $x$ such that $f_{1}(T), \ldots$, $f_{n}(T)$ are pairwise disjoint.

(b) Let $f, g \in G, T \subseteq \operatorname{var}(f) \cap \operatorname{var}(g)$ be open and $f \uparrow T \neq g \uparrow T$; then there is a nonempty open subset $T^{\prime}$ of $T$ such that $T^{\prime}, f\left(T^{\prime}\right), g\left(T^{\prime}\right)$ are pairwise disjoint.

Proof. (a) Let $T_{1}, \ldots, T_{n}$ be pairwise disjoint open neighborhoods of $f_{1}(x)$, $\ldots, f_{n}(x)$ respectively. Then $T \stackrel{\text { def }}{=} \bigcap_{i=1}^{n} f_{i}^{-1}\left(T_{i}\right)$ is as required.

(b) Let $x \in T$ be such that $f(x) \neq g(x)$. By (a) there is an open set $T_{1}$ such that $x \in T_{1} \subseteq T$ and $f\left(T_{1}\right) \cap g\left(T_{1}\right)=\varnothing$. Let $y \in T_{1}$ be such that $f(y) \neq y$. By (a) there is an open set $T_{2}$ such that $y \in T_{2} \subseteq T_{1}$ and $f\left(T_{2}\right) \cap T_{2}=\varnothing$. Let $z \in T_{2}$ be such that $g(z) \neq z$. By (a) there is an open set $T^{\prime}$ such that $z \in T^{\prime} \subseteq T_{2}$ and $g\left(T^{\prime}\right) \cap T^{\prime}=\varnothing . T^{\prime}$ is as required.

Definition 2.2. Let $\mathscr{U} \subset \tau_{X} ; \mathscr{U}$ is dense if for every $T \in \tau_{X}-\{\varnothing\}$ there is $S \in \mathscr{U}-\{\varnothing\}$ such that $S \subseteq T$. 
Definition 2.3. $M(X, G)$ is regionally disrigid if $\{\operatorname{var}(g) \mid g \in G\}$ is dense.

Assumption 2.2. $M(X, G)$ is regionally disrigid.

Lemma 2.4. (a) For every $T \in \tau_{X}-\{\varnothing\}$ and $n \in \omega$ there is $h \in Q(T)$ such that $h^{n} \neq \mathrm{Id}$. Id .

(b) Let $\varnothing \neq U \subseteq \operatorname{var}(f) \cap \operatorname{var}(g)$; then there is $h \in Q(U)$ such that $\left[f^{h}, g\right] \neq$

(c) If $T$ is open and $T \cap \operatorname{var}(f) \neq \varnothing$, then there is $h \in Q(T)$ such that $[f, h] \neq \mathrm{Id}$.

(d) If $H$ is a nontrivial normal subgroup of $G$ then $M(\operatorname{var}(H), H)$ is regionally disrigid.

Proof. (a) We prove by induction on $n \geq 1$ that for every $T \in \tau_{X}-\{\varnothing\}$ there is $h \in Q(T)$ and $x \in T$ such that for every $0 \leq i<j \leq n \quad f^{i}(x) \neq f^{j}(x)$. For $n=1$ this is just the assumption of regional disrigidity. Assume the claim is true for $n$ and we prove it for $n+1$. Let $T \in \tau_{X}-\{\varnothing\}$ and let $h, x$ be as assured by the induction hypothesis. By 2.1(a) there is an open neighborhood $T_{1}$ of $x$ such that $T_{1} \subseteq T$ and $T_{1}, h\left(T_{1}\right), \ldots, h^{n}\left(T_{1}\right)$ are pairwise disjoint. If there is $y \in T_{1}$ such that $h^{n+1}(y) \neq y$, then $h, y$ demonstrate the claim for $n+1$. Assume for every $y \in T_{1} h^{n+1}(y)=y$. Let $h^{\prime}$ be such that $\varnothing \neq \operatorname{var}\left(h^{\prime}\right) \subseteq T_{1}$, and suppose $h^{\prime}(y) \neq y$. It is easy to see that the points $y$, $h h^{\prime}(y), \ldots,\left(h h^{\prime}\right)^{n+1}(y)$ are distinct, and clearly $h h^{\prime} \in Q(T)$; hence $h h^{\prime}$ and $y$ are as required.

(b) Without loss of generality we can assume that $[f, g]=\mathrm{Id}$. We distinguish between two cases.

Case I: $f g \uparrow U \neq$ Id. Let $U_{1}=U \cap \operatorname{var}(f g)$. Since $U_{1} \subseteq \operatorname{var}(g)$ and $[g, f]=$ Id $f\left(U_{1}\right) \subseteq \operatorname{var}(g) . U_{1} \neq \varnothing$ so it follows that $f \uparrow U_{1} \neq g f\left|U_{1}=f g\right| U_{1}$. By 2.1(b) there is a nonempty open subset $T$ of $U_{1}$ such that $T, f(T)$, $f g(T)$ are pairwise disjoint. Let $T_{1}$ be a nonempty open subset of $T$ such that $g\left(T_{1}\right) \cap T_{1}=\varnothing$. Let $h \in Q\left(T_{1}\right)-\{$ Id $\}$. We show that $\left[f^{h}, g\right] \neq$ Id . Let $x \in X$ be such that $h(x) \neq x$. Note that $x, h^{-1}(x) \in T_{1}$. So $g f^{h}(x)=$ $g h f h^{-1}(x)=g f h^{-1}(x)$. On the other hand, $f^{h} g(x)=h f h^{-1} g(x)=h f g(x)=$ $f g(x)=g f(x)$. Since $h^{-1}(x) \neq x \quad g f^{h}(x) \neq f^{h} g(x)$; hence $h$ is as desired.

Case II. $f g \uparrow U=$ Id. Let $T$ be a nonempty open subset of $U$ such that $g(T) \cap T=\varnothing$. Hence also $f(T) \cap T=\varnothing$. Let $h \in Q(T)$ and $h^{2} \neq$ Id. We show that $\left[f^{h}, g\right] \neq$ Id. Let $x \in X$ be such that $h^{2}(x) \neq x$. As in the previous case it is easy to check that $f^{h} g(x)=h(x) \neq h^{-1}(x)=g f^{h}(x)$. So $h$ is as desired.

(c) This is a trivial corollary of regional disrigidity and (b).

(d) Let $\varnothing \neq U \subseteq \operatorname{var}(H)$; hence there is $f \in H$ such that $\operatorname{var}(f) \cap U \neq \varnothing$. Let $\varnothing \neq V \subseteq U$ be such that $f(V) \cap V=\varnothing$. By (c) there is $g \in Q(V)$ such that $[f, g] \neq$ Id. $[f, g]=f\left(f^{-1}\right)^{g}$, hence $[f, g] \in H . \quad \operatorname{var}([f, g]) \subseteq$ $\operatorname{var}(g)+f(\operatorname{var}(g)) \subseteq V+f(V)$. Let $h \in G$ be such that $[[f, g], h] \neq$ Id and 
$\operatorname{var}(h) \subseteq \operatorname{var}(g)$. Hence $[f, g, h] \in H$ and $\operatorname{var}([f, g, h]) \subseteq \operatorname{var}(h)+$ $[f, g](\operatorname{var}(h)) \subseteq V+[f, g](V) . \quad[f, g](V)=V$, hence $\operatorname{var}([f, g, h]) \subseteq$ $V$. Q.E.D.

The following observation will save some computation in what follows.

Observation 2.5. Let $h \in G$; then $h$ induces an automorphism $\tilde{h}$ of $M(X, G)$ : $\tilde{h}(x)=h(x)$ when $x \in X \cup \tau_{X}$ and $\tilde{h}(f)=f^{h}$ when $f \in G$.

Let $x_{1}, \ldots, x_{n}, y_{1}, \ldots, y_{n} \in M(X, G)$ and $h \in G ;$ then $\left\langle x_{1}, \ldots, x_{n}\right\rangle \stackrel{h}{\cong}$ $\left\langle y_{1}, \ldots, y_{n}\right\rangle$ means that for every $1 \leq i \leq n \tilde{h}\left(x_{i}\right)=y_{i} .\left\langle x_{1}, \ldots, x_{n}\right\rangle \cong$ $\left\langle y_{1}, \ldots, y_{n}\right\rangle$ means that there is $h$ such that $\left\langle x_{1}, \ldots, x_{n}\right\rangle \stackrel{h}{\cong}\left\langle y_{1}, \ldots, y_{n}\right\rangle$. $x \cong y$ means that $\langle x\rangle \cong\langle y\rangle$.

Let $Z(\mathbf{f}) \stackrel{\text { def }}{=}\{g \mid(\forall f \in \mathbf{f})([g, f]=\mathrm{Id})\}$. Let $C m(\mathbf{f}, \mathbf{g})$ mean that $[\mathbf{f}, \mathbf{g}]=$ $\{$ Id $\} . Z(f), C m(f, \mathbf{g})$ etc. are defined similarly.

Definition 2.6. (a) $\mathrm{f}$ is mixed on $U$, if for every $\varnothing \neq V \subseteq U$ there is $\varnothing \neq$ $W \subseteq V$ and $f \in \mathbf{f}$ such that $W \subseteq \operatorname{var}(f)$ and $f^{Q(W)} \subseteq \mathbf{f}$.

(b) $\mathbf{f}$ is mixed, if it is mixed on $\operatorname{var}(\mathbf{f})$.

Lemma 2.7. (a) If $\mathscr{U} \subseteq R(X)$ and for every $U \in \mathscr{U}$ f is mixed on $U$, then $\mathbf{f}$ is mixed on $\sum \mathscr{U}$.

(b) If $\mathbf{f}$ is mixed on $U$ and $g \in Z(\mathbf{f})$, then $\operatorname{var}(g) \cap U=\varnothing$.

(c) If $\mathbf{f}$ is mixed then $Z(\mathbf{f})=Q(X \sim \operatorname{var}(\mathbf{f}))$.

Proof. (a) is trivial, (b) is a trivial consequence of $2.4(\mathrm{~b})$, and (c) is a trivial consequence of (b).

Let us now explain the general method by which we reconstruct $R(X)$ from $G$. We would like to represent each open set $U \in R(X)$ by the subset $Q(U)$ of $G$. The difficulty is that in order to do that, we have to find a group theoretic characterization of those subsets of $G$ which have the form $Q(U)$. For $f, g \in$ $G$ let $V(f, g)=Z\left(g^{Z(f)}\right)$. It is easy to see that if $\operatorname{var}(f) \cap \operatorname{var}(g)=\varnothing$, then $V(f, g)$ has the form $Q(U)$ for some $U \in R(X)$. So we would like to find a formula $\psi(f, g)$ in the language of groups such that for every $f, g \in G G$ F $\psi[f, g]$ iff $\operatorname{var}(f) \cap \operatorname{var}(g)=\varnothing$. Unfortunately, we did not find such a formula. Instead we found a formula $\psi_{3}(f, g)$ which has the following weaker property: (a) (Theorem 2.13(a)) If $\langle X, G\rangle$ is regionally disrigid and $G \vDash \psi_{3}[f, g]$, then $\operatorname{var}(f) \cap \operatorname{var}(g)=\varnothing$; and (b) (Theorem 2.13(b)) under additional assumptions on $\langle X, G\rangle$ there are many $f$ 's and $g$ 's for which $G \vDash \psi_{3}[f, g]$. The above additional requirements will assure that

$$
\begin{aligned}
& \left\{U \in R(X) \mid(\exists f, g \in G)\left(G \vDash \psi_{3}[f, g] \wedge Q(U)=V(f, g)\right)\right\} \quad \text { generates } R(X) . \\
& \text { Let } D(U, V) \stackrel{\text { def }}{\equiv} \forall f(f(U) \cap V=\varnothing) ; \text { let } D(\mathbf{f}, \mathbf{g}) \stackrel{\text { def }}{\equiv} D(\operatorname{var}(\mathbf{f}), \operatorname{var}(\mathbf{g})) . \\
& U \preccurlyeq V \stackrel{\text { def }}{\equiv} \forall W(D(V, W) \rightarrow D(U, W)) \text {, and } \mathbf{f} \vDash \mathbf{g} \stackrel{\text { def }}{\equiv} \operatorname{var}(\mathbf{f}) \preccurlyeq \operatorname{var}(\mathbf{g}) .
\end{aligned}
$$


Let $U \approx V \stackrel{\text { def }}{=} U \preccurlyeq V \wedge V \preccurlyeq U$, and $\mathbf{f} \approx \mathbf{g} \stackrel{\text { def }}{=} \operatorname{var}(\mathbf{f}) \approx \operatorname{var}(\mathbf{g})$. Let $\operatorname{conv}(U) \stackrel{\text { def }}{=}$ $\sum\{g(U) \mid g \in G\}$ and $\operatorname{conv}(\mathbf{f}) \stackrel{\text { def }}{=} \operatorname{conv}(\operatorname{var}(\mathbf{f}))$.

Lemma 2.8. (a) Let $\varphi_{D}(\mathbf{f}, \mathbf{g})=\forall h C m\left(\mathbf{f}, \mathbf{g}^{h}\right)$; then for every $\mathbf{f}$ and $\mathbf{g}, G \vDash$ $\varphi_{D}[\mathbf{f}, \mathbf{g}]$ iff $D(\mathbf{f}, \mathbf{g})$ holds in $M(X, G)$.

(b) There are formulas $\varphi_{\preccurlyeq}(\mathbf{f}, \mathbf{g})$ and $\varphi_{\approx}(\mathbf{f}, \mathbf{g})$ in the language of groups such that for every $\mathbf{f}$ and $\mathbf{g}, G \vDash \varphi_{\preccurlyeq}[\mathbf{f}, \mathbf{g}]$ iff $\mathbf{f} \preccurlyeq \mathbf{g}$ holds in $M(X, G)$, and $G \vDash$ $\varphi_{\approx}[\mathbf{f}, \mathbf{g}]$ iff $\mathbf{f} \approx \mathbf{g}$ holds in $M(X, G)$.

Proof. (a) follows trivially from 2.4(b), and (b) follows easily from (a).

Definition 2.9. (a) Let $D\left(U_{1}, U_{2} ; V\right) \stackrel{\text { def }}{=} \forall f\left(\operatorname{var}(f) \cap V=\varnothing \rightarrow f\left(U_{1}\right) \cap U_{2}=\varnothing\right)$.

(b) Let

$$
\begin{aligned}
& \operatorname{conv}(U ; V) \stackrel{\text { def }}{=} \sum\{f(U) \mid \operatorname{var}(f) \cap V=\varnothing\}, \quad \text { and } \\
& \operatorname{conv}(U ; \mathbf{g}) \stackrel{\text { def }}{=} \sum\{f(U) \mid f \in Z(\mathbf{g})\} .
\end{aligned}
$$

(d) Let $D\left(\mathbf{f}_{1}, \mathbf{f}_{2} ; V\right) \equiv D\left(\operatorname{var}\left(\mathbf{f}_{1}\right), \operatorname{var}\left(\mathbf{f}_{2}\right) ; V\right)$ and $D\left(\mathbf{f}_{1}, \mathbf{f}_{2} ; \mathbf{g}\right)$ and $\operatorname{conv}(\mathbf{f}, \mathbf{g})$ be defined similarly.

(e) We say that $U$ is $V$-flexible if for every $W_{1}, W_{2} \subseteq U, D\left(W_{1}, W_{2} ; V\right)$ implies $D\left(W_{1}, W_{2}\right)$; we say that $U$ is g-flexible if for every $W_{1}, W_{2} \subseteq U$, $D\left(W_{1}, W_{2} ; \mathbf{g}\right)$ implies $D\left(W_{1}, W_{2}\right) . \operatorname{Fl}(U ; V)$ and $\operatorname{Fl}(U ; \mathbf{g})$ denote, respectively, that $U$ is $V$-flexible and that $U$ is g-flexible.

Note that $D\left(U_{1}, U_{2} ; \mathbf{g}\right)$ implies $D\left(U_{1}, U_{2} ; \operatorname{var}(\mathbf{g})\right)$, and $\operatorname{Fl}(U ; V) \wedge \operatorname{var}(\mathbf{g}) \subseteq$ $V$ implies $\operatorname{Fl}(U ; \mathbf{g})$.

Definition 2.10. (a) Let $V(\mathbf{f}, \mathbf{g}, \mathbf{h})=Z\left(\mathbf{g}^{Z(\mathbf{f})}\right) \cap\left\{f^{\prime} \mid f^{\prime} \preccurlyeq \mathbf{h}\right\}$.

(b) Let

$$
\psi_{1}\left(\mathbf{f}, \mathbf{f}^{\prime}\right) \equiv \forall g\left(\neg C m\left(g, \mathbf{f}^{\prime}\right) \rightarrow\left(\exists g_{1} \preccurlyeq g\right)\left(g_{1} \neq \operatorname{Id} \wedge C m\left(V\left(\mathbf{f}, g, g_{1}\right), \mathbf{f}^{\prime}\right)\right)\right) .
$$

Lemma 2.11. (a) $V(\mathbf{f}, \mathbf{g}, \mathbf{h}) \subseteq Q(\operatorname{conv}(\mathbf{h}) \sim \operatorname{conv}(\operatorname{var}(\mathbf{g}) \cap \mathbf{f i x}(\mathbf{f}) ; \mathbf{f}))$.

(b) $V(\mathbf{f}, \mathbf{g}, \mathbf{h}) \cap Q(\mathbf{f i x}(\mathbf{f}))=Q(\operatorname{conv}(\mathbf{h}) \cap \operatorname{fix}(\mathbf{f}) \sim \operatorname{conv}(\mathbf{g} ; \mathbf{f}))$.

(c) If $\operatorname{var}(\mathbf{g}) \cap \operatorname{var}(\mathbf{f}) \cap \operatorname{conv}(\mathbf{h})=\varnothing$, then

$$
V(\mathbf{f}, \mathbf{g}, \mathbf{h})=Q(\operatorname{conv}(\mathbf{h}) \sim \operatorname{conv}(\mathbf{g} ; \mathbf{f})) .
$$

(d) If $G \vDash \psi_{1}[\mathbf{f}, \mathbf{f}]$, then $\mathrm{Fl}(\operatorname{var}(\mathbf{f}) ; \mathbf{f})$ holds in $M(X, G)$. In particular, if $G \vDash \psi_{1}\left[\mathbf{f}, \mathbf{f}^{\mathbf{f}}\right]$, then $D\left(\operatorname{var}\left(\mathbf{f}^{\prime}\right) \cap \operatorname{var}(\mathbf{f}), \operatorname{var}\left(\mathbf{f}^{\prime}\right) \cap \mathbf{f i x}(\mathbf{f})\right)$ holds in $M(X, G)$.

(e) If $\operatorname{var}\left(\mathbf{f}^{\prime}\right) \cap \operatorname{var}(\mathbf{f})=\varnothing$ and $\mathrm{Fl}\left(\operatorname{var}\left(\mathbf{f}^{\prime}\right)\right.$;f) holds in $M(X, G)$, then $G \vDash$ $\psi_{1}\left[\mathbf{f}, \mathbf{f}^{\prime}\right]$.

Proof. (a), (b), (c) are trivial consequences of 2.4(b) and 2.7.

(d) Suppose $\neg \mathrm{Fl}\left(\operatorname{var}\left(\mathbf{f}^{\prime}\right) ; \mathbf{f}\right)$, and we show that $G \not \psi_{1}\left[\mathbf{f}, \mathbf{f}^{\prime}\right]$. Let $W_{1}, W_{2} \subseteq$ $\operatorname{var}\left(\mathbf{f}^{\prime}\right)$ be such that $W_{1} \cong W_{2}$, but $D\left(W_{1}, W_{2} ; \mathbf{f}\right)$. Since $M(X, G)$ is regionally disrigid, by $2.4(\mathrm{c})$ there is $g \in G$ such that $\operatorname{var}(g) \subseteq W_{1}$ and $\neg C m\left(g, \mathbf{f}^{\prime}\right)$. Let $g_{1} \preccurlyeq g$ and $g_{1} \neq$ Id. We show that $\neg C m\left(V\left(\mathbf{f}, g, g_{1}\right), \mathbf{f}^{\prime}\right)$ holds. 
Let $W_{3}=W_{2} \cap \operatorname{conv}\left(g_{1}\right)$. Clearly $W_{3} \neq \varnothing . \quad \operatorname{var}\left(g^{Z(f)}\right) \cap W_{3}=\varnothing$, hence $Z\left(g^{Z(\mathfrak{f})}\right)$ is mixed on $W_{3}$, i.e., $V\left(f, g, g_{1}\right)$ is mixed on $W_{3}$. Hence since $\operatorname{var}\left(\mathbf{f}^{\prime}\right) \cap W_{3} \neq \varnothing$, by $2.7(\mathbf{b}) \neg C m\left(V\left(\mathbf{f}, g, g_{1}\right), \mathbf{f}^{\prime}\right)$ holds. So (d) is proved.

(e) Suppose $\operatorname{var}\left(\mathbf{f}^{\prime}\right) \cap \operatorname{var}(\mathbf{f})=\varnothing$ and $\mathrm{Fl}\left(\operatorname{var}\left(\mathbf{f}^{\prime}\right) ; \mathbf{f}\right)$ holds. Suppose $\neg C m\left(g, \mathbf{f}^{\prime}\right)$ holds. Hence $\operatorname{var}(g) \cap \operatorname{var}(\mathbf{f}) \neq \varnothing$. Let $g_{1} \neq$ Id be such that $\operatorname{var}\left(g_{1}\right) \subseteq$ $\operatorname{var}(g) \cap \operatorname{var}\left(\mathbf{f}^{\prime}\right)$. By (a) and (c):

$$
\begin{aligned}
V\left(\mathbf{f}, g, g_{1}\right) & \subseteq Q\left(\operatorname{conv}\left(g_{1}\right) \sim \operatorname{conv}(\operatorname{var}(g) \cap \text { fix }(\mathbf{f}) ; \mathbf{f})\right) \\
\subseteq Q\left(\operatorname{conv}\left(g_{1}\right)\right. & \left.\sim \operatorname{conv}\left(\operatorname{var}\left(g_{1}\right) ; f\right)\right) .
\end{aligned}
$$

Since $\operatorname{var}\left(g_{1}\right) \subseteq \operatorname{var}\left(\mathbf{f}^{\prime}\right)$ and $\mathrm{Fl}\left(\operatorname{var}\left(\mathbf{f}^{\prime}\right) ; \mathbf{f}\right)$ holds, $\operatorname{var}\left(\mathbf{f}^{\prime}\right) \cap \operatorname{conv}\left(g_{1}\right)=$ $\operatorname{var}\left(\mathbf{f}^{\prime}\right) \cap \operatorname{conv}\left(g_{1} ; \mathbf{f}\right)$, and hence $\operatorname{var}\left(\mathbf{f}^{\prime}\right) \cap\left(\operatorname{conv}\left(g_{1}\right) \sim \operatorname{conv}\left(g_{1} ; \mathbf{f}\right)\right)=\varnothing$; hence $\mathbf{f}^{\prime} \subseteq Z\left(V\left(\mathbf{f}, g, g_{1}\right)\right)$. This shows that $G \vDash \psi_{1}\left[\mathbf{f}, \mathbf{f}^{\prime}\right]$.

Lemma 2.12. Let $k_{0}, \ldots, k_{n} \in \omega$ and suppose that $f^{k_{0}}(V), \ldots, f^{k_{n}}(V)$ are pairwise disjoint; then for every $h_{1}, \ldots, h_{n} \in Z(f)$ and $\varnothing \neq W \subseteq V$ $\sum_{i=0}^{n} f^{k_{i}}(W) \nsubseteq \sum_{i=1}^{n} h_{i}(V)$.

Proof. By induction on $n . n=1$ : Suppose by contradiction that for some $h_{1} \in Z(f)$ and $\varnothing \neq W \subseteq V \quad h_{1}(V) \supseteq f^{k_{0}}(W)+f^{k_{1}}(W)$. Hence $\varnothing \neq W \subseteq$ $f^{-k_{0}}\left(h_{1}(V)\right) \cap f^{-k_{1}}\left(h_{1}(V)\right)$. Since $\langle V, f\rangle \cong\left\langle h_{1}(V), f\right\rangle, f^{-k_{0}}(V) \cap f^{-k_{1}}(V) \neq$ $\varnothing$. So $f^{k_{1}}(V) \cap f^{k_{0}}(V)=f^{k_{0}+k_{1}}\left(f^{-k_{0}}(V) \cap f^{-k_{1}}(V)\right) \neq \varnothing$; this contradicts our assumption, so the case $n=1$ is proved.

Suppose the claim has been proved for $n \geq 1$ and we prove it for $n+1$. Let $f, k_{0}, \ldots, k_{n+1}, V, h_{1}, \ldots, h_{n+1}$, and $W$ be as in the claim. By applying the induction hypothesis to $f, k_{0}, \ldots, k_{n}, V, h_{1}, \ldots, h_{n}$, and $W$ we conclude that there is $\varnothing \neq W_{1} \subseteq W$ and $0 \leq l \leq n$ such that $\sum_{i=1}^{n} h_{i}(V) \cap f^{k_{l}}\left(W_{1}\right)=\varnothing$. Now we replace $k_{l}$ by $k_{n+1}$; hence by applying the induction hypothesis to $f$, $k_{0}, \ldots, k_{l-1}, k_{l+1}, \ldots, k_{n}, k_{n+1}, V, h_{1}, \ldots, h_{n}$, and $W_{1}$ we conclude that there is $\varnothing \neq W_{2} \subseteq W_{1}$ and $m \in\{0, \ldots, l-1, l+1, \ldots, n+1\}$ such that $\sum_{i=1}^{n} h_{i}(V) \cap f^{k_{m}}\left(W_{2}\right)=\varnothing$. Now we apply the case $n=1$ to $f, k_{l}, k_{m}, V$, $h_{n+1}$, and $W_{2}$; hence $h_{n+1}(V) \nsupseteq f^{k_{l}}\left(W_{2}\right)+f^{k_{m}}\left(W_{2}\right)$. Since $R(X)$ is a Boolean algebra it follows that $\sum_{i=1}^{n+1} h_{i}(V) \nsupseteq \sum_{i=0}^{n+1} f^{k_{i}}(W)$, and the lemma is proved.

Let

$$
\begin{aligned}
\psi_{2}\left(f, f^{\prime}\right) \equiv \forall h\left([h, f] \neq \operatorname{Id} \rightarrow\left(\exists f_{1}, f_{2} \in Z\left(f^{\prime}\right)\right)\right. \\
\left.\left(\left[h, f_{1}, f_{2}\right] \neq \operatorname{Id} \wedge \psi_{1}\left(\left[h, f_{1}, f_{2}\right], f^{\prime}\right)\right)\right) .
\end{aligned}
$$

Let

$$
\begin{aligned}
\psi_{3}\left(f, f^{\prime}\right) \equiv\left(\forall g \preccurlyeq f^{\prime}\right)((g & \neq \mathrm{Id}) \rightarrow\left(\exists f^{\prime \prime} \preccurlyeq g\right) \\
\left(\left(f^{\prime \prime}\right)^{12}\right. & \left.\left.\neq \operatorname{Id} \wedge \psi_{1}\left(f,\left\{f^{\prime}, f^{\prime \prime}\right\}\right) \wedge \psi_{2}\left(f, f^{\prime \prime}\right)\right)\right) .
\end{aligned}
$$

Theorem 2.13. (a) If $\operatorname{var}(f) \cap \operatorname{var}\left(f^{\prime}\right) \neq \varnothing$, then $G \not \psi_{3}\left[f, f^{\prime}\right]$.

(b) If $\mathrm{Fl}\left(\operatorname{var}\left(f^{\prime}\right) ; \operatorname{var}(f)\right)$, then $G \vDash \psi_{3}\left[f, f^{\prime}\right]$. 
Proof. (a) We first prove the following claim.

Claim 1. If $\operatorname{var}\left(f^{\prime \prime}\right) \subseteq \operatorname{var}(f)$ and $\left(f^{\prime \prime}\right)^{12} \neq$ Id, then $G \not \models \psi_{2}\left[f, f^{\prime \prime}\right]$.

Proof. Since $\left(f^{\prime \prime}\right)^{12} \neq$ Id there is $x \in X$ such that $x, f^{\prime \prime}(x),\left(f^{\prime \prime}\right)^{2}(x)$, $\left(f^{\prime \prime}\right)^{3}(x),\left(f^{\prime \prime}\right)^{4}(x)$ are distinct. By 2.1(a) there is an open neighborhood $T$ of $x$ such that $T, f^{\prime \prime}(T),\left(f^{\prime \prime}\right)^{2}(T),\left(f^{\prime \prime}\right)^{3}(T),\left(f^{\prime \prime}\right)^{4}(T)$ are pairwise disjoint. $T \subseteq \operatorname{var}\left(f^{\prime \prime}\right) \subseteq \operatorname{var}(f)$, so by $2.4(\mathrm{c})$ there is $h$ such that $[h, f] \neq$ Id and $U \stackrel{\text { def }}{=} \operatorname{var}(h) \subseteq T$. Let $f_{1}, f_{2} \in Z\left(f^{\prime \prime}\right)$. We denote $\left[h, f_{1}, f_{2}\right]=g$. Suppose $g \neq$ Id, and we shall show that $\psi_{1}\left(g, f^{\prime \prime}\right)$ cannot hold.

(1)

$$
\begin{aligned}
g & =\left[h, f_{1}, f_{2}\right]=\left[h \cdot\left(h^{-1}\right)^{f_{1}}, f_{2}\right]=h \cdot\left(h^{-1}\right)^{f_{1}} \cdot\left(\left(h \cdot\left(h^{-1}\right)^{f_{1}}\right)^{-1}\right)^{f_{2}} \\
& =h \cdot\left(h^{-1}\right)^{f_{1}} \cdot h^{f_{2} f_{1}} \cdot\left(h^{-1}\right)^{f_{2}} .
\end{aligned}
$$

From (1) it follows that

(2) $\operatorname{var}(g) \subseteq U+f_{1}(U)+f_{2} f_{1}(U)+f_{2}(U)$.

Since $U \subseteq \operatorname{var}\left(f^{\prime \prime}\right)$ and $f_{1}, f_{2} \in Z\left(f^{\prime \prime}\right)$, and by (2)

(3) $\operatorname{var}(g) \subseteq \operatorname{var}\left(f^{\prime \prime}\right)$.

By 2.12 using (2) it follows that for every $\varnothing \neq U_{1} \subseteq U \sum_{i=0}^{4}\left(f^{\prime \prime}\right)^{i}\left(U_{1}\right) \sim$ $\left(U_{1}+f_{1}\left(U_{1}\right)+f_{2} f_{1}\left(U_{1}\right)+f_{2}\left(U_{1}\right)\right) \neq \varnothing$. Hence conv $(\operatorname{var}(g)) \subseteq \operatorname{conv}(U) \subseteq$ $\operatorname{conv}\left(\sum_{i=0}^{4}\left(f^{\prime \prime}\right)^{i}(U) \sim\left(U+f_{1}(U)+f_{2} f_{1}(U)+f_{2}(U)\right)\right) \subseteq \operatorname{conv}\left(\operatorname{var}\left(f^{\prime \prime}\right) \sim \operatorname{var}(g)\right)$, namely

(4) $\operatorname{var}(g) \preccurlyeq \operatorname{var}\left(f^{\prime \prime}\right) \sim \operatorname{var}(g)$.

By (3), (4), and the fact that $g \neq \operatorname{Id}, \neg D\left(\operatorname{var}\left(f^{\prime \prime}\right) \cap \operatorname{var}(g), \operatorname{var}\left(f^{\prime \prime}\right) \cap \mathrm{fix}(g)\right)$ holds, hence by 2.11 (d) $G \not \neq \psi_{1}\left[g, f^{\prime \prime}\right]$. We have thus proved Claim 1 .

We are now ready to prove (a). Suppose $\operatorname{var}(f) \cap \operatorname{var}\left(f^{\prime}\right) \neq \varnothing$, and let $\varnothing \neq \operatorname{var}(g) \subseteq \operatorname{var}(f) \cap \operatorname{var}\left(f^{\prime}\right)$. Hence Id $\neq g \preccurlyeq f^{\prime}$; we shall show that there is no $f^{\prime \prime}$ as required in $\psi_{3}$.

Let $f^{\prime \prime} \preccurlyeq g,\left(f^{\prime \prime}\right)^{12} \neq \mathrm{Id}$, and $G \vDash \psi_{1}\left[f,\left\{f^{\prime}, f^{\prime \prime}\right\}\right]$; we shall show that $\operatorname{var}\left(f^{\prime \prime}\right) \subseteq \operatorname{var}(f)$, and hence it will follow from Claim 1 that $G \not \psi_{2}\left[f, f^{\prime \prime}\right]$.

Suppose by contradiction $\operatorname{var}\left(f^{\prime \prime}\right) \cap \operatorname{fix}(f) \neq \varnothing$; then

$$
\varnothing \neq \operatorname{var}\left(f^{\prime \prime}\right) \cap \operatorname{fix}(f) \preccurlyeq \operatorname{var}\left(f^{\prime \prime}\right) \preccurlyeq \operatorname{var}(g) \preccurlyeq \operatorname{var}\left(f^{\prime}\right) \cap \operatorname{var}(f) .
$$

Hence $\neg D\left(\operatorname{var}\left(\left\{f^{\prime \prime}, f^{\prime}\right\}\right) \cap \operatorname{var}(f), \operatorname{var}\left(\left\{f^{\prime \prime}, f^{\prime}\right\}\right) \cap \operatorname{fix}(f)\right)$. By $2.11(\mathrm{~d}) G \not \neq$ $\psi_{1}\left[f,\left\{f^{\prime}, f^{\prime \prime}\right\}\right]$, a contradiction. Hence $\operatorname{var}\left(f^{\prime \prime}\right) \subseteq \operatorname{var}(f)$, so by Claim 1 $G \not \forall \psi_{2}\left[f, f^{\prime \prime}\right]$. This proves (a).

(b) We first prove the following claim.

Claim 2. Suppose $\mathrm{Fl}\left(\operatorname{var}\left(f^{\prime}\right) ; \operatorname{var}(f)\right)$, then $G \vDash \psi_{2}\left[f, f^{\prime}\right]$.

Proof. Let $h$ be such that $[h, f] \neq$ Id. Hence there is $\varnothing \neq U \subseteq \operatorname{var}(f)$ such that $h(U) \cap U=\varnothing$. Let $f_{1} \in Q(U)-\{$ Id $\}$. We check that $\operatorname{var}\left(\left[h, f_{1}\right]\right) \subseteq$ $U+h(U),\left[h, f_{1}\right](U)=U$, and $U \cap \operatorname{var}\left(\left[h, f_{1}\right]\right) \neq \varnothing$.

(1) $\left[h, f_{1}\right]=h f_{1} h^{-1} f_{1}^{-1}=f_{1}^{h} f_{1}^{-1}$. 
Hence $\operatorname{var}\left(\left[h, f_{1}\right]\right) \subseteq h\left(\operatorname{var} f_{1}\right)+\operatorname{var}\left(f_{1}^{-1}\right) \subseteq h(U)+U . \quad\left[h, f_{1}\right] \backslash U=$ $f_{1}^{-1} \uparrow U$ and hence $\operatorname{var}\left(\left[h, f_{1}\right]\right) \cap U \neq \varnothing$ and $\left[h, f_{1}\right](U)=U$. Let $\varnothing \neq$ $U_{1} \subseteq U$ be such that $\left[h, f_{1}\right]\left(U_{1}\right) \cap U_{1}=\varnothing$, and let $f_{2} \in Q\left(U_{1}\right)-\{$ Id $\}$. It follows from the same argument as above that $\left[h, f_{1}, f_{2}\right]=\left[\left[h, f_{1}\right], f_{2}\right] \neq$ Id and that $\operatorname{var}\left(\left[h, f_{1}, f_{2}\right]\right) \subseteq \operatorname{var}\left(f_{2}\right)+\left[h, f_{1}\right]\left(\operatorname{var}\left(f_{2}\right)\right) \subseteq U+U=U$. Hence $\operatorname{var}\left(\left[h, f_{1}, f_{2}\right] \subseteq \operatorname{var}(f)\right.$. Since by assumption $\operatorname{Fl}\left(\operatorname{var}\left(f^{\prime}\right) ; \operatorname{var}(f)\right)$, it follows that $G \vDash \psi_{1}\left(\left[h, f_{1}, f_{2}\right], f^{\prime}\right]$. Also since $\operatorname{var}\left(f_{1}\right), \operatorname{var}\left(f_{2}\right)$ are disjoint from $\operatorname{var}\left(f^{\prime}\right), f_{1}, f_{2} \in Z\left(f^{\prime}\right)$, hence $G \vDash \psi_{2}\left[f, f^{\prime}\right]$ and Claim 2 is proved.

We are now ready to prove (b). Suppose $\mathrm{Fl}\left(\operatorname{var}\left(f^{\prime}\right)\right.$; $\left.\operatorname{var}(f)\right)$ holds, and let Id $\neq g \preccurlyeq f^{\prime}$. By $2.4($ a $)$ there is $f^{\prime \prime} \preccurlyeq g$ such that $\operatorname{var}\left(f^{\prime \prime}\right) \subseteq \operatorname{var}\left(f^{\prime}\right)$ and $\left(f^{\prime \prime}\right)^{12} \neq$ Id. By Claim $2 G \vDash \psi_{2}\left[f, f^{\prime \prime}\right] ;$ and since $\mathrm{Fl}\left(\operatorname{var}\left(\left\{f^{\prime}, f^{\prime \prime}\right\}\right) ; \operatorname{var}(f)\right)$ holds $G \vDash \psi_{1}\left[f,\left\{f^{\prime}, f^{\prime \prime}\right\}\right]$. It follows that $G \vDash \psi_{3}\left[f, f^{\prime}\right]$, so (b) is proved.

Let $C(X, G)=\left\{U \mid(\exists f, g, h \in G)\left(\exists G_{1} \subseteq G\right)\left(G_{1}\right.\right.$ is a normal subgroup of $G, f, g \in G_{1}, G_{1} \vDash \psi_{3}[f, g]$, and $\left.Q(U)=V(f, g, h)\right\}$.

Assumption 2.3. $C(X, G)$ generates $R(X)$. (That is, $R(X)$ is the smallest complete subalgebra of $R(X)$ which contains $C(X, G)$.)

Let $K^{0}=\left\{M(X, G) \mid M(X, G)\right.$ satisfy Assumptions 2.1-2.3\}. Let $K_{0}^{0}=$ $\left\{G \mid \exists X\left(G \subseteq H(X) \wedge M(X, G) \in K^{0}\right)\right.$ and let $K_{1}^{0}=\{H R(X, G) \mid M(X, G) \in$ $\left.K^{0}\right\}$.

Theorem 2.14. (a) $K_{1}^{0}$ is second-order interpretable in $K_{0}^{0}$.

(b) Let $M\left(X_{i}, G_{i}\right) \in K^{0}, i=1,2$, and $\varphi$ be an isomorphism between $G_{1}$ and $G_{2}$; then $\varphi$ can be uniquely extended to an isomorphism between $H R\left(X_{1}, G_{1}\right)$ and $H R\left(X_{2}, G_{2}\right)$.

Remark. Even though Assumption 2.3 is not very explicit the reader can notice that the following property $(*)$ of $M(X, G)$ already implies that $M(X, G) \in$ $K^{0}:(*) \quad\{U \mid M(X, G) \vDash \operatorname{Fl}(U ; \sim U)\}$ is dense in $\tau_{X}$. Most classes which will be considered later have property $(*)$. For instance, in $\mathbf{R}^{n}$ every open ball and even every regular open connected set $U$ satisfy $\operatorname{Fl}(U ; \sim U)$.

Proof of 2.14. (a) The official definition of interpretation requires that we find second-order formulas $\varphi_{U}, \varphi_{\mathrm{Eq}}, \varphi_{\complement}$, and $\varphi_{\mathrm{Op}}$ to represent the universe of $H R(X, G)$, the equality, and the other relations on $H R(X, G)$. We will be somewhat informal and will leave it to reader to check some of the details.

Each element of $G$ will be represented by itself. Each $U \in R(X)$ will be represented by $Q(U)$. We need a formula $\psi_{R}(\mathrm{f})$ such that $G \vDash \psi_{R}[\mathrm{f}]$ iff for some $U \in R(X) \quad \mathbf{f}=Q(U)$. Let $\psi_{4}(\mathbf{f}) \equiv(\exists f, g, h)\left(\exists G_{1} \subseteq G\right) \quad\left(\left(G_{1}\right.\right.$ is a normal subgroup of $\left.\left.G) \wedge\left(f, g \in G_{1}\right) \wedge\left(G_{1} \vDash \psi_{3}[f, g]\right) \wedge \mathbf{f}=V(f, g, h)\right)\right)$. It is easy to see that $\psi_{4}(f)$ is indeed a second-order formula. We show that for every $\mathbf{f} \subseteq G, G \vDash \psi_{4}[\mathbf{f}]$ iff for some $U \in C(X, G), \mathbf{f}=Q(U)$. By the definition of $C(X, G)$, for every $U \in C(X, G) \quad G \vDash \psi_{4}[Q(U)]$. Suppose $G \vDash \psi_{4}[\mathrm{f}]$ and let $f, g, h$, and $G_{1}$ be as assured by $\psi_{4}$. By Lemma 2.4(d) 
$M\left(\operatorname{var}\left(G_{1}\right), G_{1}\right)$ is regionally disrigid, hence by $2.13(\mathrm{a}) \operatorname{var}(f) \cap \operatorname{var}(g)=\varnothing$. Hence by 2.11 (c) $V(f, g, h)=Q(\operatorname{conv}(h) \sim \operatorname{conv}(g ; f))$. So $\mathbf{f}=Q(U)$ where $U=\operatorname{conv}(h) \sim \operatorname{conv}(g ; f)$. Hence for some $U \in C(X, G), \mathbf{f}=Q(U)$.

Note that if $\mathscr{U} \subseteq R(X)$ and $\Pi(\mathscr{U})=\operatorname{int}(\bigcap \mathscr{U})$ is the meet of $\mathscr{U}$ in $R(X)$, then $Q(\Pi(\mathscr{U}))=\bigcap\{Q(U) \mid U \in \mathscr{U}\}$, and if $U \in R(X)$ then $Q(\sim U)=$ $Z(Q(U))$. So since $C(X, G)$ generates $R(X)$, for every $\mathbf{f} \subseteq G$ : there is $U$ such that $\mathbf{f}=Q(U)$ iff $\mathbf{f}$ can be obtained from $\{Q(U) \mid U \in C(X, G)\}$ by repeated application of the operations $Z$ and $\cap$. It is thus clear that $H R(X, G)$ is reconstructible from $G$, but in order to satisfy the formal definition of interpretability, we have still to show that there is a second-order formula $\psi_{R}(\mathbf{f})$ which says that $\mathbf{f}$ can be obtained in the above way.

It is well known that the derivation of an element from other elements using certain operations can be described by means of evaluated trees. We briefly describe this formalism.

A well-founded tree is a partially ordered set $\langle T, \leq\rangle$ such that for every $x \in T\{y \mid y \leq x\}$ is linearly ordered and such that every set of pairwise comparable elements is finite. For our present needs let us define a $G$-evaluated tree.

Definition. A $G$-evaluated tree is a well-founded tree $T$ together with a function $E: T \rightarrow P(G) \cup\{\bigcap, Z\}$ such that for every $x \in T, E(x) \in P(G)$ iff $x$ is maximal in $T$, and whenever $x \in T$ has more than one successor, then $E(x)=\bigcap$. Let $\langle T, E\rangle$ be a $G$-evaluated tree; then it is easy to define by transfinite induction the value $v_{T, E}(x)$ for every $x \in T: v_{T, E}(x)=E(x)$ iff $x$ is a maximal element of $T ; v_{T, E}(x)=Z\left(v_{T, E}(y)\right)$ if $E(x)=Z$ and $y$ is the unique successor of $x$; and $v_{T, E}(x)=\bigcap\left\{v_{T, E}(y) \mid y\right.$ is a successor of $\left.x\right\}$ if $E(x)=\bigcap$.

It is easy to see that $\{Q(V) \mid V \in R(X)\}=\{\mathbf{h} \mid$ there is a $G$-evaluated tree $\langle T, E\rangle$ and $x \in T$ such that (1) $|T| \leq|G|$; (2) for every $y \in T$ if $E(y) \in$ $P(G)$ then $\psi_{4}(E(y))$ holds; and $\left.(3) \mathbf{h}=v_{T, E}(x)\right\}$. It is thus easy to see that $\{Q(V) \mid V \in R(X)\}$ is second-order definable in $G$.

The operations $\circ, \subseteq$, Op are trivially definable, so the theorem is proved.

Question 1. Is $\{H R(X, G) \mid M(X, G)$ is Hausdorff and regionally disrigid $\}$ interpretable in $\{G \mid \exists X(G \subseteq H(X) \wedge M(X, G)$ is Hausdorff and regionally disrigid) $\}$ ?

Our next goal is to obtain a first-order interpretation; naturally we do not know how to do it without the strengthening of Assumption 2.3. On the other hand, it is obvious that a first-order interpretation of all $H R(X, G)$ is impossible because of cardinality considerations; for take a countable subgroup $G$ of $H(\mathbf{R})$ such that $M(\mathbf{R}, G)$ is regionally disrigid. Every structure which is first-order interpretable in $G$ has cardinality $\leq \aleph_{0}$, whereas $|H R(\mathbf{R}, G)|=2^{\aleph_{0}}$. This observation implies that we have to be more modest in what we want to interpret.

Let $V(X, G)=\{\operatorname{var}(f) \mid f \in G\}$ and $H V(X, G)=\langle G, V(X, G) ; \circ, \subseteq$, Op). Our goal is to obtain a first-order interpretation of $H V(X, G)$ in $G$. 
(Since we have assumed regional disrigidity, $H R(X, G)$ is second-order interpretable in $H V(X, G)$.)

In fact we tried, without success, to prove a stronger and a neater theorem than what is presented here. This is the first-order version of Question 1. So the following question remains open.

Question 2. If $\{H V(X, G) \mid M(X, G)$ is Hausdorff and regionally disrigid $\}$ firstorder interpretable in $\{G \mid \exists X(G \subseteq H(X) \wedge M(X, G)$ is Hausdorff and regionally disrigid)\}?

For a while we drop Assumption 2.3 and assume that $M(X, G)$ satisfies only Assumptions 2.1 and 2.2 ; that is, $M(X, G)$ is Hausdorff and regionally disrigid.

Our plan is to find a first-order formula in the language of groups $\chi(f, g)$ which is equivalent to the fact that $\operatorname{var}(f) \cap \operatorname{var}(g)=\varnothing$. From such a $\chi$ it is easy to obtain a first-order interpretation of $H V(X, G)$ in $G$; for let $\chi_{\subseteq}(f, g)=\forall h(\chi(h, g) \rightarrow \chi(h, f))$. Clearly $\chi_{\subseteq}(f, g)$ is equivalent to the fact that $\operatorname{var}(f) \subseteq \operatorname{var}(g)$. The desired interpretation is then obtained very easily.

To make our way of thinking clearer we shall first consider a $\chi$ that works just for a rather special case. Then we shall present our theorem in its full generality; its form will be the following: Let $K$ be the class of $M(X, G)$ 's having the property $\Phi,(\Phi$ will be defined later); then $\{H V(X, G) \mid M(X, G) \in K\}$ is first-order interpretable in $\{G \mid \exists X(G \subseteq H(X) \wedge M(X, G) \in K)\}$.

The property $\Phi$ that defines $K$ is not very transparent, but we shall show that the class $K$ is quite big. This will be done in two ways: first we shall show that $K$ is closed under certain operations, and second we shall show that many large natural classes of $M(X)$ 's and $M(X, G)$ 's happen to be included in $K$.

We start with one special case. Recall that $\psi_{3}(f, g)$ is a first-order formula. 2.13 tells us two facts about $\psi_{3}:(1) \psi_{3}(f, g)$ does not hold if $\operatorname{var}(f) \cap$ $\operatorname{var}(g) \neq \varnothing ;(2)$ there are some cases in which $\psi_{3}(f, g)$ does hold, namely when $\mathrm{Fl}(\operatorname{var}(g) ; \operatorname{var}(f))$ holds. It turns out that for 0-dimensional spaces $\mathrm{Fl}(U ; V)$ is equivalent to $U \cap V=\varnothing$, so for such spaces $\psi_{3}(f, g)$ holds iff $\operatorname{var}(f) \cap \operatorname{var}(g)=\varnothing$. The precise statement is given in the following proposition.

Proposition 2.15. Suppose $M(X, G)$ has the following properties: (1) every nonempty open set contains a nonempty clopen set; (2) if $g \in G, V$ is clopen, and $g(V) \cap V=\varnothing$, then $g_{V} \stackrel{\text { def }}{=} g\left|V \cup\left(g^{-1} \mid g(V)\right) \cup \operatorname{Id}\right|(X-V-g(V)) \in G$. Then for every $U, V \in R(X), \operatorname{Fl}(U ; V)$ holds in $M(X, G)$ iff $U \cap V=\varnothing$; and therefore for every $f, g \in G, G \vDash \psi_{3}[f, g]$ iff $\operatorname{var}(f) \cap \operatorname{var}(g)=\varnothing$.

We leave the trivial proof to the reader.

In order to deal with the general case in which a first-order interpretation can be obtained, we need some definitions. 
We define by induction properties $\mathrm{Fl}_{n}^{i}(U ; V)$ where $i=0,1,2$ and $n \in \omega$.

$$
\begin{aligned}
\mathrm{Fl}_{0}^{0}(U ; V) & \equiv \mathrm{Fl}_{0}^{1}(U ; V) \equiv \mathrm{Fl}(U ; V), \\
\mathrm{Fl}_{2 n+1}^{0}(U ; V) & \equiv \sum\left\{U^{\prime} \subseteq U \mid \mathrm{Fl}_{2 n}^{0}\left(U^{\prime} ; V\right)\right\}=U, \\
\mathrm{Fl}_{2 n+2}^{0}(U ; V) & \equiv \sum\left\{V^{\prime} \subseteq V \mid \mathrm{Fl}_{2 n+1}^{0}\left(U ; V^{\prime}\right)\right\}=V, \\
\mathrm{Fl}_{2 n+1}^{1}(U ; V) & \equiv \sum\left\{V^{\prime} \subseteq V \mid \mathrm{Fl}_{2 n}^{1}\left(U ; V^{\prime}\right)\right\}=V, \quad \text { and } \\
\mathrm{Fl}_{2 n+2}^{1}(U ; V) & \equiv \sum\left\{U^{\prime} \subseteq U \mid \mathrm{Fl}_{2 n+1}^{1}\left(U^{\prime} ; V\right)\right\}=U . \\
\mathrm{Fl}_{0}^{2}(U ; V) & \equiv \mathrm{Fl}(U ; V) \wedge \mathrm{Fl}_{(V ; U), \quad \text { and }} \\
\mathrm{Fl}_{n+1}^{2}(U ; V) & \equiv \sum\left\{V^{\prime} \subseteq V \mid \sum\left\{U^{\prime} \subseteq U \mid \mathrm{Fl}_{n}^{2}\left(U^{\prime} ; V^{\prime}\right)\right\}=U\right\}=V .
\end{aligned}
$$

Let $\Phi_{n}^{i} \equiv \forall U \mathrm{Fl}_{n}^{i}(U ; \sim U)$ and $\Phi_{n} \equiv \Phi_{n}^{0} \vee \Phi_{n}^{1}$. Clearly $\Phi_{n}^{i}$ implies $\Phi_{n+1}^{i}$. One can construct examples of $M(X, G)$ 's that show that the $\Phi_{n}^{i}$ 's are not equivalent. In fact, the natural classes of $M(X, G)$ 's to which we shall apply our general theorem will all satisfy $\Phi_{1}^{0}$.

We shall use the following trivial observation. If $\mathrm{Fl}_{n}^{i}(U ; V), U^{\prime} \subseteq U$, and $V^{\prime} \subseteq V$ hold, then $\mathrm{Fl}_{n}^{i}\left(U^{\prime} ; V^{\prime}\right)$ holds.

Let us define the following sequence of first-order formulas: $\chi_{0}(f, g) \equiv$ $\psi_{3}(f, g)$. Suppose $\chi_{n}(f, g)$ has been defined; then

$$
\chi_{n}^{+}(f, g) \equiv Z\left(\left\{f^{\prime} \mid \chi_{n}\left(f^{\prime}, g\right)\right\}\right) \subseteq Z(f)
$$

and

$$
\chi_{n+1} \equiv Z\left(\left\{g^{\prime} \mid \chi_{n}^{+}\left(f, g^{\prime}\right)\right\}\right) \subseteq Z(g) .
$$

Lemma 2.16. (a) If $\operatorname{var}(f) \cap \operatorname{var}(g) \neq \varnothing$, then for every $n \in \omega: G \vDash \neg \chi_{n}[f, g]$.

(b) If $\mathrm{Fl}_{n}^{2}(\operatorname{var}(f)$; $\operatorname{var}(g))$ holds in $M(X, G)$, then $G \vDash \chi_{n}[f, g]$.

(c) If $i=0$ or $i=1$ then $\mathrm{Fl}_{n}^{i}(U ; V) \wedge \mathrm{Fl}_{n}^{i}(V ; U)$ implies $\mathrm{Fl}_{n}^{2}(U ; V)$.

Proof. (a) By induction on $n$. For $n=0$ the claim is proved in 2.13(a). Suppose the claim is true for $n$. Let $\operatorname{var}(f) \cap \operatorname{var}(g) \neq \varnothing$, and let $h$ be such that $\operatorname{var}(h) \subseteq \operatorname{var}(f) \cap \operatorname{var}(g)$ and $[h, f] \neq$ Id. By the induction hypothesis for every $f^{\prime}$ such that $G \vDash \chi_{n}\left[f^{\prime}, g\right]: \operatorname{var}\left(f^{\prime}\right) \cap \operatorname{var}(h)=\varnothing$. Hence $h \in$ $Z\left(\left\{f^{\prime} \mid G \vDash \chi_{n}\left[f^{\prime}, g\right]\right\}\right)$ but $h \notin Z(f)$. So $G \not k \chi_{n}^{+}[f, g]$. We have thus proved that if $\operatorname{var}(f) \cap \operatorname{var}(g) \neq \varnothing$, then $G \not \models \chi_{n}^{+}[f, g]$. In the same way it is proved that if $\operatorname{var}(f) \cap \operatorname{var}(g) \neq \varnothing$, then $G \not \models \chi_{n+1}[f, g]$.

(b) By induction on $n$. The case $n=0$ is proved in 2.13(b). Suppose the claim is true for $n$, and let $\mathrm{Fl}_{n+1}^{2}(\operatorname{var}(f)$; $\operatorname{var}(g))$ hold in $M(X, G)$. By the regional disrigidity and the observation preceding 2.16

$$
\begin{aligned}
\sum\left\{\operatorname{var}\left(g^{\prime}\right) \subseteq \operatorname{var}(g) \mid \sum\left\{\operatorname{var}\left(f^{\prime}\right) \subseteq \operatorname{var}(f) \mid \mathrm{Fl}_{n}^{2}\left(\operatorname{var}\left(f^{\prime}\right) ; \operatorname{var}\left(g^{\prime}\right)\right)\right\}=\right. & \operatorname{var}(f)\} \\
& =\operatorname{var}(g) .
\end{aligned}
$$

It follows easily from the definitions that $G \vDash \chi_{n+1}[f, g]$. 
(c) By induction on $n$. For $n=0$ the claim is true by the definitions. Suppose the claim is true for $2 n$. Let $\mathrm{Fl}_{2 n+1}^{0}(U ; V)$ and $\mathrm{Fl}_{2 n+1}^{0}(V ; U)$ hold in $M(X, G)$. Let $A=\left\{U^{\prime} \subseteq U \mid \mathrm{Fl}_{2 n}^{0}\left(U^{\prime}, V\right)\right.$ holds $\}$ and $B=\left\{V^{\prime} \subseteq V \mid\right.$ $\mathrm{Fl}_{2 n}^{0}\left(V^{\prime} ; U\right)$ holds $\}$. So $\sum A=U$ and $\sum B=V$. By the observation preceding this lemma for every $U^{\prime} \in A$ and $V^{\prime} \in B \mathrm{Fl}_{2 n}^{0}\left(U^{\prime} ; V^{\prime}\right)$ and $\mathrm{Fl}_{2 n}^{0}\left(V^{\prime} ; U^{\prime}\right)$ hold, and thus by the induction hypothesis $\mathrm{Fl}_{2 n}^{2}\left(U^{\prime}, V^{\prime}\right)$ holds. So $\sum\left\{V^{\prime} \subseteq V\right.$ | $\left.\sum\left\{U^{\prime} \subseteq U \mid \mathrm{Fl}_{2 n}^{2}\left(U^{\prime} ; V^{\prime}\right)\right\}=U\right\}=V$, that is, $\mathrm{Fl}_{2 n+1}^{2}(U ; V)$ holds. The argumentation is similar in the three remaining cases.

Let $K^{(n)}=\{M(X, G) \mid M(X, G)$ satisfy Assumptions 2.1, 2.2 and $M(X, G)$ $\left.\vDash \Phi_{n}\right\}$. Note that $K^{(n)} \subseteq K^{(n+1)} \subseteq K^{0}$. Let $K_{0}^{(n)}=\{G \mid \exists X(G \subseteq H(X) \wedge$ $\left.\left.M(X, G) \in K^{(n)}\right)\right\}$, and let $K_{2}^{(n)}=\left\{H V(X, G) \mid M(X, G) \in K^{(n)}\right\}$.

Corollary 2.17. $K_{2}^{(n)}$ is first-order interpretable in $K_{0}^{(n)}$.

Proof. By 2.16, if $M(X, G) \in K^{(n)}$, then for every $f, g \in G \quad G \vDash \chi_{n}[f, g]$ iff $\operatorname{var}(f) \cap \operatorname{var}(g)$. The remaining details in the interpretation are as explained in the discussion that follows Question 2.

There is one interesting case in which $V(X, G)=R(X)$; we now consider this case.

Lemma 2.18. If $M(X, H(X))$ is regionally disrigid and $X$ is second countable, then $V(X, H(X))=R(X)$.

Proof. Let $\left\{T_{n} \mid n \in \omega\right\}$ be a base for $X$, and let $V \in R(X)$. We define by induction a sequence $\left\{f_{n} \mid n \in \omega\right\}$ of elements of $H(X)$. Suppose $f_{0}, \ldots, f_{n-1}$ have been defined $n \geq 0$; if $V \cap T_{n}=\varnothing$ define $f_{n}=$ Id. Suppose $V \cap T_{n} \neq \varnothing$. If for some $k<n \quad V \cap T_{n} \cap \operatorname{var}\left(f_{k}\right) \neq \varnothing$, define $f_{n}=$ Id. Otherwise let $\sigma_{n} \subseteq\{0, \ldots, n-1\}$ be a maximal subset such that $V \cap T_{n} \cap \bigcap_{k \in \sigma_{n}} T_{k} \neq \varnothing$. Let $f_{n}$ be such that

$$
\varnothing \neq \operatorname{var}\left(f_{n}\right) \subseteq V \cap T_{n} \cap \bigcap_{k \in \sigma_{n}} T_{k} .
$$

Let $f=\bigcup_{n \in \omega}\left(f_{n}\left\lceil\operatorname{var}\left(f_{n}\right)\right) \cup \mathrm{Id} \uparrow\left(X-\bigcup_{n \in \omega} \operatorname{var}\left(f_{n}\right)\right)\right.$. We show that $f \in$ $H(X)$. The only nontrivial fact is that $f$ and $f^{-1}$ are continuous. Since $X$ is second countable it is sufficient to show that if $\lim x_{k}=x$, then $\lim f\left(x_{k}\right)=$ $f(x)$. Suppose $\left\{x_{k}\right\}$ is a counterexample. Without loss of generality there is a 1-1 sequence $n_{k}$ such that $x_{k} \in \operatorname{var}\left(f_{n_{k}}\right), \lim \left(x_{k}\right)=x$, and there is $i$ such that $x \in T_{i}$ and for every $k f\left(x_{k}\right) \notin T_{i}$. Let $k^{\prime}$ be such that $x_{k^{\prime}} \in T_{i}$ and $n_{k^{\prime}}>i . \quad x_{k^{\prime}} \in T_{i} \cap \operatorname{var}\left(f_{n_{k^{\prime}}}\right)$. By the maximality of $\sigma_{n_{k}}, i \in \sigma_{n_{k}}$, and hence $\operatorname{var}\left(f_{n_{k^{\prime}}}\right) \subseteq T_{i}$. But $T_{i} \not \supset f\left(x_{k^{\prime}}\right)=f_{n_{k^{\prime}}}\left(x_{k^{\prime}}\right) \in \operatorname{var}\left(f_{n_{k^{\prime}}}\right) \subseteq T_{i}$, a contradiction.

So $f$ is continuous, and similarly $f^{-1}$ is.

It is easy to see that $\operatorname{var}(f)=V$, so the lemma is proved.

Question 3. Is 2.18 true for every regionally disrigid metric space? 
The main results of this section are 2.14 and 2.17; these theorems however are formulated in an abstract way. In the remainder of this section we shall apply the above theorems to some concrete classes of $M(X, G)$ 's.

Definition 2.19. Let $X$ and $Y$ be topological spaces and $h: X \rightarrow Y$ be a homeomorphism of $X$ into $Y . h$ is a correct embedding of $X$ into $Y$, if $h(X)$ is open, and $\bigcup\{T \subseteq X \mid T$ is open and $\operatorname{cl}(h(T)) \subseteq h(X)\}$ is dense in $X$.

Proposition 2.20. (a) Let $Y$ be a regular space and $h: X \rightarrow Y$ be a homeomorphism; then $h$ is a correct embedding iff $h(X)$ is open.

(b) Let $h: X \rightarrow Y$ be correct, $f \in H(X)$, and $\operatorname{cl}(h(\operatorname{var}(f))) \subseteq h(X)$. Let $\tilde{f}=h f h^{-1} \cup \operatorname{Id}\lceil(Y-h(X))$; then $\tilde{f} \in H(Y)$.

Proof. Trivial.

Definition 2.21. Let $K$ be a class of $M(X, G)$ 's. We say that $M(Y, H)$ is regionally $K$-formed if there is a family $\left\{\left\langle T_{i}, h_{i}\right\rangle \mid i \in I\right\}$ such that (1) for every $i$ there is $M\left(X_{i}, G_{i}\right) \in K$ such that $T_{i} \subseteq X_{i}$ is open, $h_{i}: T_{i} \rightarrow Y$ is correct, and for every $f \in G_{i}$ : if $\operatorname{var}(f) \subseteq T_{i}$ and $\operatorname{cl}\left(h_{i}(\operatorname{var}(f))\right) \subseteq h_{i}\left(T_{i}\right)$, then $\tilde{f} \stackrel{\text { def }}{=} h_{i} f h_{i}^{-1} \cup \operatorname{Id} \uparrow\left(Y-h_{i}\left(T_{i}\right)\right) \in H$; and (2) $\bigcup\left\{h_{i}\left(T_{i}\right) \mid i \in I\right\}$ is dense in $Y$.

We denote by $\operatorname{Reg}(K)$ the class of all $M(X, G)$ 's which are regionally $K$ formed. If $K$ is a class of topological spaces, then $\operatorname{Reg}(K)$ is the class of $Y$ 's such that $M(Y, H(Y))$ is regionally $\{M(X, H(X)) \mid X \in K\}$-formed. Note that if $K$ is the class of all $M(X, G)$ 's which are regionally disrigid, then $K=\operatorname{Reg}(K)$.

Of special interest is the class $K^{*}=\left\{M(X, G) \mid M(X, G) \vDash \Phi_{1}^{0}\right\} . K^{*} \subseteq K^{(1)}$ so 2.17 applies to $K^{*} . K^{*}$ is interesting for the following reasons: (a) It already contains most of the natural classes of $M(X, G)$ 's to which we know to apply 2.14; (b) $K^{*}=\operatorname{Reg}\left(K^{*}\right)$, and hence showing that a certain $M(X, G) \in K^{*}$ implies automatically that many other $M(X, G)$ 's belong to $K^{*}$.

Proposition 2.22. (a) If $M(X, G) \vDash "\{U \mid \mathrm{Fl}(U ; \sim U)\}$ is dense in $\tau_{X}$ ", then $M(X, G) \in K^{*}$.

(b) $K^{*}=\operatorname{Reg}\left(K^{*}\right)$.

Proof. Easy.

Some nontopological faithfulness results can be inferred from 2.17. They are presented in the following theorem.

Theorem 2.23.1 [R2]. (a) Let $K^{B}=\{B \mid B$ is a complete Boolean algebra and for every $b \in B-\{0\}$ there is $g \in \operatorname{Aut}(B)$ such that $g \neq \mathrm{Id}$, and for every $a \in B$ such that $a \wedge b=0 \quad g(a)=a\}$. Then $\left\{\langle B, \operatorname{Aut}(B) ; \leq, 0, \mathrm{Op}\rangle \mid B \in K^{B}\right\}$ is first-order interpretable in $\left\{\operatorname{Aut}(B) \mid B \in K^{B}\right\}$.

(b) Let $K^{M}=\left\{\langle B, \mu\rangle \mid\langle B, \mu\rangle\right.$ is a measure algebra and $\left.\mu\left(1_{B}\right)=1\right\}$. Let $\operatorname{Aut}(B, \mu)$ be the group of measure-preserving automorphisms of $B$. Then 
$\left\{\left\langle\operatorname{Aut}(B, \mu), B,[0,1] ; \mu, \leq_{B},+_{[0,1]}, \circ, \mathrm{Op}\right\rangle \mid\langle B, \mu\rangle \in K^{M}\right\}$ is first-order interpretable in $\left\{\operatorname{Aut}(B, \mu) \mid\langle B, \mu\rangle \in K^{M}\right\}$. (See [En] for related results which are obtained by a different method.)

(c) $A$ tree $T$ is a poset such that for every $a, b, c \in T$ if $a, b \leq c$ then $a$ and $b$ are comparable $(a \diamond b)$. Let $K^{T}$ be the class of all trees $T$ that have the following properties: (1) $T$ has a root $O_{T}$, i.e., for every $a \in T O_{T} \leq a$; (2) for every $a \in T$ there is $b \in T$ such that $a<b$; (3) for every $a, b \in T$ if $a<b$, then there is $c \in T$ such that $a<c$ and $c$ is incomparable with $b(c \diamond b) ;(4)$ every $a, b \in T$ have a greatest lower bound $c(c=a \wedge b) ;(5)$ if $a, b, c \in T$ are distinct and $a \wedge b=c$, then there is $a^{\prime} \in T$ such that $a^{\prime} \leq a$ and $a^{\prime}$ is a successor of $c$ (i.e., there is no $d$ such that $c<d<a^{\prime}$ ); we denote $a^{\prime}$ by $S_{a}(c) ;(6)$ every nonempty subset $A \subseteq T$ has a greatest lower bound denoted by $\wedge A$; and (7) for every $a, b \in T$ there is $f \in \operatorname{Aut}(T)$ such that $f(a) \diamond b$ and $f(a \wedge b)=a \wedge b$.

Note that (1)-(5) are first-order requirements, (6) is a completeness requirement, and (7) is a homogeneity requirement. $\{\langle\operatorname{Aut}(T), T ; \leq, \circ, \mathrm{Op}\rangle \mid T \in$ $\left.K^{T}\right\}$ is first-order interpretable in $\left\{\operatorname{Aut}(T) \mid T \in K^{T}\right\}$.

(d) [GGHJ] For a linear ordering $\langle L,<\rangle$ let $\operatorname{Bet}(<)$ be the betweenness three-place relation determined by $<$. Let $\bar{L}$ denote the Dedekind completion of $L$. Let $K^{L}$ be the class of all linear orderings $\langle L,<\rangle$ that have the following property: there is a dense subset $D \subseteq L$ such that for every $a_{1}<b_{1}, a_{2}<b_{2}$ in $D$ there is $f \in \operatorname{Aut}(\langle L,<\rangle)$ such that $f\left(a_{1}\right)=a_{2}$ and $f\left(b_{1}\right)=b_{2}$. Then $\left\{\langle\operatorname{Aut}(\langle L,<\rangle), \bar{L} ; \operatorname{Bet}(<), \circ, \mathrm{Op}\rangle \mid\left\langle L,\langle\rangle \in K^{L}\right\}\right.$ is first-order interpretable in $\left\{\operatorname{Aut}\left(\langle L,\langle\rangle) \mid\left\langle L,\langle\rangle \in K^{L}\right\}\right.\right.$.

Remarks. In (a), (b), (c), and (d) we did not present the strongest possible formulation; our main purpose was rather to demonstrate the range of applications that Theorems 2.14 and 2.17 have.

Proof. A result stronger than (a) appears in [R2] and a result stronger than (d) appears in [GGHJ]; however, both (a) and (d) are trivial corollaries of 2.17. Using Maharam's theorem [Mm], (b) is also a trivial corollary of 2.17.

We prove (c). Let $T \in K^{T}$. Let $B(T)=\{b \subseteq T \mid b$ is a maximal set of pairwise comparable elements $\}$. Let $\mathscr{B}(T)=\left\{V_{a} \mid a \in T\right\}$ where $V_{a}=\{b \in B(T) \mid a \in b\} . \mathscr{B}(T)$ is a base consisting of clopen sets for a 0-dimensional topology on $B(T)$, and it is easy to check that by requirement (7) $M(B(T), \operatorname{Aut}(T))$ is regionally disrigid and moreover for every $a \in T$ $M(B(T), \operatorname{Aut}(T)) \vDash \mathrm{Fl}\left(V_{a} ; \sim V_{a}\right)$. Let $R(T) \stackrel{\text { def }}{=} R(B(T))$; hence by 2.17 and 2.22(a) $M_{1}(T) \stackrel{\text { def }}{=}\langle\operatorname{Aut}(T), R(T) ; \subseteq, \circ, \mathrm{Op}\rangle$ is interpretable in $\operatorname{Aut}(T)$.

Let $\varphi_{1}(V) \equiv\left(\forall U_{1}, U_{2} \subseteq V\right)$ (if $U_{1} \neq \varnothing \neq U_{2}$, then there is $g$ such that $g\left(U_{1}\right) \cap U_{2} \neq \varnothing, g \uparrow(\sim V)=\mathrm{Id}$, and $\left.g^{2}=\mathrm{Id}\right)$. Note that $\varphi_{1}(V)$ is a first-order formula in the language of $M_{1}(T)$. We show that for every $V \in M_{1}(T): M_{1}(T) \vDash \varphi_{1}[V]$ iff $(*)$ there is $a \in T$ such that (1) for every 
$b \in T$ if $V_{b} \subseteq V$ then $a \leq b$; and (2) for every $b, c \in T$ if $V_{b} \subseteq V$ and $a<c<b$, then $V_{c} \subseteq V$.

Suppose that $V$ satisfies $(*)$ and let $U_{1}, U_{2} \subseteq V$ be nonempty. Without loss of generality $U_{1}=V_{b}, U_{2}=V_{c}$, and $b \not \Delta c$. Let $g_{1} \in \operatorname{Aut}(T)$ be such that $g_{1}(b)$ is comparable with $c$ and $g_{1}(b \wedge c)=b \wedge c$. Let $g: T \rightarrow T$ be defined as follows: For every $d \in T$ : if $b \wedge c<d \wedge b$, then $g(d)=g_{1}(d)$; if $b \wedge c<d \wedge c$, then $g(d)=g_{1}^{-1}(d)$; otherwise $g(d)=d$. Clearly $g \in \operatorname{Aut}(T)$ and $g$ is as required in $\varphi_{1}$.

Suppose $M_{1}(T) \vDash \varphi_{1}[V]$. Let $a=\bigwedge\left\{b \mid V_{b} \subseteq V\right\}$ and we show that $a$ is as required in (*). Clearly if $V_{b} \subseteq V$, then $a \leq b$. Suppose by contradiction $V_{b} \subseteq V, a<c<b$ but $V_{c} \nsubseteq V$. Hence $V_{c} \cap \sim V \neq \varnothing$ and hence for some $d>c \quad V_{d} \cap V=\varnothing$. Let $e \in T$ be such that $V_{e} \subseteq V$ and $b \wedge e<c$; such an $e$ exists because $c>\wedge\left\{t \mid V_{t} \subseteq V\right\}$. Let $g \in \operatorname{Aut}(T)$ be such that $g^{2}=$ Id and $g\left(V_{b}\right) \cap V_{e} \neq \varnothing$; we show that $g(d) \not d$ and hence $g \backslash(\sim V) \neq$ Id. By replacing $b$ by $b^{\prime}>b$ and $e$ by $e^{\prime}>e$ we can w.l.o.g. assume that $g(b)=e$. Hence $g(b \wedge e)=g(b) \wedge g(e)=e \wedge b$, and hence $g(c)>g(b \wedge e)=b \wedge e$. $g(c)<g(b)=e$, so $b \wedge e<g(c)<e$ and so $g(c) \not>c$, but this implies that $g(d) \not d$. Hence $g \backslash(\sim V) \neq \mathrm{Id}$ and $(*)$ is proved.

Let $\varphi_{2}(V) \equiv V \neq \varnothing \wedge \varphi_{1}(V) \wedge \forall U\left(\varphi_{1}(U) \rightarrow((U \subseteq V) \vee(V \subseteq U) \vee(U \cap\right.$ $V=\varnothing())$ ). We show that $M_{1}(T) \vDash \varphi_{2}[V]$ iff for some $a \in T \quad V=V_{a}$. Clearly if $V=V_{a}$, then $M_{1}(T) \vDash \varphi_{2}[V]$. Suppose $M_{1}(T) \vDash \varphi_{2}[V]$. Let $a=$ $\bigwedge\left\{b \mid V_{b} \subseteq V\right\}$ and we show that $V=V_{a}$. By definition $V \subseteq V_{a}$. Suppose by contradiction $b>a$ but $V_{b} \nsubseteq V$. Let $c$ be such that $V_{c} \subseteq V$; hence since $V$ satisfies $\varphi_{1} \quad b \wedge c=a$. Without loss of generality $b=S_{b}(a)$ and $c=S_{c}(a)$. Let $d$ be such that $V_{d} \subseteq V$ and $c \wedge d=a$. Hence $V_{b} \cup V_{c}$ satisfies $\varphi_{1}$ but $V_{b} \cup V_{c}$ is neither comparable nor is it disjoint from $V$. Hence $V$ does not satisfy $\varphi_{2}$, a contradiction.

It is now clear how to interpret $\langle\operatorname{Aut}(T), T ; \leq, \mathrm{Op}\rangle$ in $M_{1}(T)$, hence (c) is proved.

We make the convention that if $X_{0}$ is a topological space and $K$ is a class of $M(X, G)$ 's, then $X_{0} \in K$ means that $M\left(X_{0}, H\left(X_{0}\right)\right) \in K$.

We shall now list some classes of $M(X, G)$ 's which are included in $K^{*}$. The proofs are mostly trivial or easy so they are left to the reader.

Proposition 2.23.2. (a) For every $n \mathbf{R}^{n} \in K^{*}$, and hence every regionally Euclidean space belongs to $K^{*}$. Note that this class contains manifolds with boundary and polyhedra.

(b) If $Y$ is a locally convex Hausdorff topological vector space over $\mathbf{R}$, then $Y \in K^{*}$.

(c) If $Y$ is a normed vector space over an ordered field, then $Y \in K^{*}$.

(d) If $k \leq \infty X$ is a $k$ times continuously differentiable manifold, $C^{k}(X)$ is the group of $k$ times continuously differentiable nonsingular homeomorphisms of $X$, and $G \supseteq C^{k}(X)$, then $M(X, G) \in K^{*}$. Many other variants of the above 
belong to $K^{*} ;$ e.g., add a volume form and consider those homeomorphisms which preserve volume, replace "continuously differentiable" by "differentiable."

(e) If $P L(X)$ is the group of PL-homeomorphisms of a PL manifold $X$, and $G \supseteq P L(X)$, then $M(X, G) \in K^{*}$.

(f) If $X$ is a normed vector space over an ordered field $F, L(X)$ is the group of all homeomorphisms $f$ such that both $f$ and $f^{-1}$ are Lipschitz, and $G \supseteq L(X)$, then $M(X, G) \in K^{*}$.

(g) Let $X$ be a manifold and $F$ be an equivalence relation on $X$ such that for every $x \in X$ there is a neighborhood $U$ of $x$ such that $\langle U, F \mid U\rangle$ is isomorphic to $\left\langle\mathbf{R}^{n}, F_{n, k}\right\rangle$ where $F_{n, k}=\left\{\left\langle\left(x_{1}, \ldots, x_{n}\right),\left(y_{1}, \ldots, y_{n}\right)\right\rangle\right.$ for every $\left.i \leq k, x_{i}=y_{i}\right\}$.

We call $\langle X, F\rangle$ a manifold with a simple foliation. Let $H(X, F)$ be the group of all homeomorphisms $f$ of $X$ which preserve $F$, that is, $\langle x, y\rangle \in F$ iff $\langle f(x), f(y)\rangle \in F$. Let $H_{0}(X, F)=\{f \mid f \in H(X)$ and for all $x \in X$ $\langle x, f(x)\rangle \in F\}$. Then $M\left(X, H_{0}(X, F)\right) \in K^{*}$ and $M(X, H(X, F)) \in K^{0}$.

The smooth versions of the above are also in $K^{*}$ and $K^{0}$, respectively.

(h) Let $\langle L,<\rangle$ be a linearly ordered set; we denote the group of automorphisms of $\langle L,<\rangle$ by $\operatorname{Aut}(\langle L,<\rangle)$. Note that $\operatorname{Aut}(\langle L,<\rangle)$ is a subgroup of $H(L)$ where $L$ is considered together with its order topology. Let $K^{O R}=$ $\{M(L, \operatorname{Aut}(\langle L,<\rangle)) \mid\langle L,<\rangle$ is a linear ordering and $M(L, \operatorname{Aut}(L,<))$ is regionally disrigid $\}$; then $K^{O R} \subseteq K^{*}$. If $\langle L,<\rangle$ is a dense linear ordering such that $M(L, \operatorname{Aut}(\langle L,<\rangle))$ is regionally disrigid and for some $c \in$ $L\{f(c) \mid f \in \operatorname{Aut}(\langle L,<\rangle)\}$ is dense in $L$, then for every $G$ such that $\operatorname{Aut}(\langle L,<\rangle) \subseteq G \subseteq H(L): M(L, G) \in K^{*}$.

(i) If $\lambda$ is an infinite cardinal, then $[0,1]^{\lambda} \in K^{*}$.

(j) If $\bar{F}$ is the Dedekind completion of an ordered field $F$, then for every cardinal $\lambda \bar{F}^{\lambda} \in K^{*}$.

(k) If $X$ is 0-dimensional and regionally disrigid then $X \in K^{*}$.

(1) Let $C$ denote the Cantor set, $\mathbf{Q}$ be the rationals, I the irrationals, and $\mathbf{R}$ the reals; then any product of these spaces belongs to $K^{0}$. More generally if $X \in K^{*}$ and $Y$ is 0-dimensional, then $X \times Y \in K^{0}$.

(m) Let $\kappa$ be an infinite cardinal and for every $i \in I$, let $X_{i}$ be a Euclidean manifold. Let $Y=\prod_{i=I}^{\kappa} X_{i}$ be the space whose points are the elements of $\prod_{i \in I} X_{i}$ and whose basic open sets are $\prod_{i \in I} U_{i}$ where each $U_{i}$ is open in $X_{i}$ and $\left|\left\{i \mid U_{i} \neq X_{i}\right\}\right|<\kappa$. Then $Y \in K^{0}$. If $Y$ is as above, $\lambda$ is an infinite cardinal and $x \in Y$, let $Y^{\lambda}(x)=\{y \in Y||\{i \mid x(i) \neq y(i)\} \mid<\lambda\}$; then the subspace $Y^{\lambda}(x)$ belongs to $K^{0}$.

(n) Let $\langle T,<\rangle$ be a tree; for every $a \in T$ let $X_{a}$ be a topological space. Let $Y=\prod_{a \in T}^{T} X_{a}$ be the following space: a point of $Y$ is a function $x$ from some $b \in B(T)$ to $\bigcup_{a \in T} X_{a}$ such that for every $a \in b \quad x(a) \in X_{a}$. A basic open set of $Y$ has the form $\prod_{a \in b} U_{a}$ where $b \in B(T)$, for every $a \in b, U_{a}$ is open in 
$X_{a}$, and for some $a_{0} \in b, U_{a}=X_{a}$ for every $a \geq a_{0}$. If each $X_{a}$ is a manifold, then $Y \in K^{0}$.

\section{THE RECONSTRUCTION OF $X$ FROM $H R(X, G)$}

In this section we prove four results all having more or less the following form. We define a certain class $K$ of $M(X, G)$ 's, and then, letting $K_{1}$ be $\{H R(X, G) \mid M(X, G) \in K\}$ and $K_{3}$ be $\left\{\left\langle X, \tau_{X} ; \in\right\rangle \mid \exists G(M(X, G) \in K)\right\}$, we prove that $K_{3}$ is interpretable in $K_{1}$ and that every isomorphism between elements $H R\left(X_{i}, G_{i}\right)$ of $K_{1}, i=1,2$, is induced by a homeomorphism between $X_{i}, i=1,2$. Each of the three first results extends [W] in some natural direction. We withdraw all assumptions of $\S 2$.

I. Locally compact spaces

Our first result is the easiest for the following reasons: (a) we consider only locally compact $M(X, G)$ 's; (b) we assume relatively strong homogeneity requirements; and (c) we do not try to obtain a first-order interpretation and are ready to accept any interpretation.

Nevertheless, our class of $M(X, G)$ 's contains $\{M(X) \mid X$ is a Euclidean manifold $\},\left\{M\left(X, C^{k}(X)\right) \mid X\right.$ is a differential manifold of $n$th order and $k \leq n \leq \infty\}$, and $\{M(X, P L(X)) \mid X$ is a $P L$-manifold and $P L(X)$ is the group of piecewise linear homeomorphisms of $X\}$.

Definition 3.1. (a) Let $x \in X, x$ is densely conjugated in $M(X, G)$ if $\{f(x) \mid$ $f \in G\}$ is somewhere dense in $X$, that is, $\operatorname{int}(\operatorname{cl}(\{f(x) \mid f \in G\})) \neq \varnothing$. Let $D C(X, G)$ denote the set of densely conjugated points of $M(X, G)$.

(b) $M(X, G)$ has property $D$ if for every distinct $x, y \in D C(X, G)$ : if $y \in \operatorname{cl}(\{f(x) \mid f \in G\})$, then $\{f(x) \mid f \in G$ and $f(y)=y\}$ is somewhere dense.

(c) Let $\operatorname{HRP}(X, G)=\langle H R(X, G), X ; \in, O p\rangle$, where $\in$ is the belonging relation between elements of $X$ and elements of $R(X)$, and $\langle f, x, y\rangle \in \mathrm{Op}$ iff $f \in G, x, y \in X$, and $f(x)=y$. Let $H R D(X, G), H V P(X, G)$, and $H V D(X, G)$ be respectively the submodels of $H R P(X, G)$ with universes $R(X) \cup D C(X, G), H V(X, G) \cup X$, and $H V(X, G) \cup D C(X, G)$.

Note that if $R(X)$ is an open base for $X$, then $\operatorname{HRP}(X, G)$ determines $X$; if $X$ is regular then $R(X)$ is an open base; if $M(X, G)$ is regionally disrigid then $\langle V(X, G), \subseteq\rangle$ determines $\langle R(X), \subseteq\rangle$.

Definition 3.2. (a) $X$ is regionally compact if $\bigcup\{T \mid T$ is open and $\operatorname{cl}(T)$ is compact $\}$ is dense in $X$.

(b) $K^{R D}=\{M(X, G) \mid X$ is regionally compact Hausdorff space and $M(X, G)$ has property $D\}$. Let $K_{1}^{R D}=\left\{H R(X, G) \mid M(X, G) \in K^{R D}\right\}$ and $K_{3}^{R D}=\left\{H R D(X, G) \mid M(X, G) \in K^{R D}\right\}$.

Theorem 3.3. (a) $K_{3}^{R D}$ is interpretable in $K_{1}^{R D}$. 
(b) Let $\varphi: H R\left(X_{1}, G_{1}\right) \rightarrow H R\left(X_{2}, G_{2}\right)$ be an isomorphism between two elements of $K_{1}^{R D}$; then $\varphi$ has a unique extension to an isomorphism between $\operatorname{HRD}\left(X_{1}, G_{1}\right)$ and $\operatorname{HRD}\left(X_{2}, G_{2}\right)$.

Remarks. (a) Note that local compactness implies regional compactness.

(b) The reader could now see that combining 3.3 with 2.13 one gets already many interesting reconstructibility results; that is, for many $M(X, G)$ 's we can already conclude that $G_{1} \cong G_{2}$ implies $X_{1} \cong X_{2}$.

Proof. (b) will follow from the proof of (a). Let us explain how. $H R(X, G)$ is a submodel of $H R D(X, G)$. In our interpretation of $H R D(X, G)$ in $H R(X, G)$ every element $c$ of $H R D(X, G)$ that belongs to $H R(X, G)$ will be represented by $c$ itself. Let $\varphi$ be an isomorphism between two $H R(X, G)$ 's. Clearly $\varphi$ induces an isomorphism $\tilde{\varphi}$ between the corresponding $H R D(X, G)$ 's; but since each element of $H R(X, G)$ is represented by itself, for every $c \in H R(X, G)$ $\tilde{\varphi}(c)=\varphi(c)$, so $\tilde{\varphi}$ extends $\varphi$. The uniqueness is trivial.

Now we survey the proof of (a). Let $S(X)$ be the set of ultrafilters of $R(X)$. (Recall that $R(X)$ is a Boolean algebra.) Since $X$ is Hausdorff, for every $p \in S(X), \bigcap\{\operatorname{cl}(V) \mid V \in p\}$ contains at most one point; we denote this point by $x_{p}$ if it exists. Let $p \in S(X)$; we call $p$ a good ultrafilter if there is $U \in p$ such that for every nonempty $V \subseteq U$ there is $V^{\prime} \cong V$ such that $V^{\prime} \in p$. Clearly, if $X$ is regionally compact and $p$ is good, then $\bigcap\{\operatorname{cl}(V) \mid V \in p\} \neq \varnothing$; so if $M(X, G) \in K^{R D}$ and $p$ is good, then $x_{p}$ exists. Second, it is clear from the definition of being good that this notion is expressible in terms of $H R(X, G)$. Third, we show that if $X$ is regionally compact and $p$ is good then $x_{p} \in D C(X, G)$. Suppose not, and let $U$ be as assured by the goodness of $p$. Without loss of generality $\operatorname{cl}(U)$ is compact. Let $\left.y \in U-\operatorname{cl}\left\{f\left(x_{p}\right) \mid f \in G\right\}\right)$. It is easy to see that there is an open set $T$ such that $y \in T \subseteq \operatorname{cl}(T) \subseteq$ $X-\operatorname{cl}\left(\left\{f\left(x_{p}\right) \mid f \in G\right\}\right)$. Let $V=\operatorname{int}(\operatorname{cl}(T)) \cap U$. Clearly for no $V^{\prime} \cong V$ $V^{\prime} \in p$, hence $p$ is not good, a contradiction.

It is also clear that if $x \in D C(X, G)$ and $p$ is an ultrafilter containing $\{U \mid x \in U\}$, then $p$ is good.

It is obvious that we mean to represent the points of $D C(X, G)$ by good ultrafilters, but we still have to express equality in terms of the relations of $H R(X, G)$; i.e., we look for a formula $\varphi_{\mathrm{Eq}}(p, q)$ in the language of $H R(X, G)$ such that for every good $p$ and $q H R(X, G) \vDash \varphi_{\mathrm{Eq}}[p, q]$ iff $x_{p}=x_{q}$. Property $D$ was devised in order to ensure the existence of such a $\varphi_{\mathrm{Eq}}$. It is easy to see that $\neg \varphi_{\mathrm{Eq}}(p, q) \stackrel{\text { def }}{\equiv}(\exists U \in p)\left(\forall U^{\prime} \in q\right)\left(U \not U^{\prime}\right) \vee(\exists U \in p)(\exists V \in q)(U \cap V=$ $\left.\varnothing \wedge\left(\forall U_{1} \subseteq U\right)\left(U_{1} \neq \varnothing \rightarrow(\exists f \in G)\left(V \in f(q) \wedge U_{1} \in f(p)\right)\right)\right)$ is as required.

We did not yet finish, since we now need a formula $\varphi_{\in}(p, U)$ to express the fact that $x_{p} \in U . \varphi_{\in}(p, U) \stackrel{\text { def }}{\equiv} \forall q\left(\left((q\right.\right.$ is $\left.\left.\operatorname{good}) \wedge \varphi_{\mathrm{Eq}}(p, q)\right) \rightarrow U \in q\right)$. $\varphi_{\mathrm{Op}}(f, p, q) \stackrel{\text { def }}{\equiv} \varphi_{\mathrm{Eq}}(f(p), q)$. This completes the proof of the theorem. 
Corollary 3.4. Let $K^{R D 1}=K^{R D} \cap K^{0}$, let $K_{0}^{R D 1}=\{G \mid \exists X(G \subseteq H(X) \wedge$ $\left.\left.M(X, G) \in K^{R D 1}\right)\right\}$, and let $K_{3}^{R D 1}=\left\{H R D(X, G) \mid M(X, G) \in K^{R D 1}\right\}$; then (a) $K_{3}^{R D 1}$ is interpretable in $K_{0}^{R D 1}$, and (b) if $M\left(X_{i}, G_{i}\right) \in K^{R D 1}, i=1,2$, and $\varphi: G_{1} \rightarrow G_{2}$ is an isomorphism between $G_{1}$ and $G_{2}$, then there is a unique isomorphism $\tilde{\varphi}$ between $\operatorname{HRD}\left(X_{1}, G_{1}\right)$ and $\operatorname{HRD}\left(X_{2}, G_{2}\right)$ which extends $\varphi$.

Proof. Combine 2.13 and 3.3.

Corollary 3.4 is a final result for some $M(X, G)$ 's, namely those for which $D C(X, G)=X$. For if $M(X, G) \in K^{R D}$ and $C D(X, G)=X$, then $X$ is locally compact and hence is regular and therefore $R(X)$ is an open base for $X$; in such a case $\operatorname{HRD}(X, G)$ determines $\left\langle X, \tau_{X}\right\rangle$. We make this statement precise in the following corollary.

Corollary 3.5. Let $K^{R D 2}=K^{R D 1} \cap\{M(X, G) \mid C D(X, G)=X\}$, let $K_{0}^{R D 2}=$ $\left\{G \mid \exists X\left(G \subseteq H(X) \wedge M(X, G) \in K^{R D 2}\right)\right\}$; then (a) $K^{R D 2}$ is interpretable in $K_{0}^{R D 2}$, and

(b) if $M\left(X_{i}, G_{i}\right) \in K^{R D 2}, i=1,2$, and $\varphi: G_{1} \rightarrow G_{2}$ is an isomorphism between $G_{1}$ and $G_{2}$, then there is a unique isomorphism between $M\left(X_{1}, G_{1}\right)$ and $M\left(X_{2}, G_{2}\right)$ extending $\varphi$. (So every isomorphism between $G_{1}$ and $G_{2}$ is induced by a homeomorphism between $X_{1}$ and $X_{2}$.)

(c) Let $K^{L C}=\{M(X, G) \mid X$ is a locally compact Hausdorff space, and for every open $T$ and $x \in T\{f(x) \mid f \in G$ and $\operatorname{var}(f) \subseteq T\}$ is somewhere dense\}; then $K^{L C} \subseteq K^{R D 2}$.

Proof. (a) is a special case of 3.4. (b) follows from the particular method of interpretation. To prove (c) it is sufficient to observe that $K^{L C} \subseteq K^{(1)}$ (see the definition of $K^{(1)}$ before 2.16). Clearly if $M(X, G) \in K^{L C}$ then $\left.\forall U\left\{\sum U^{\prime} \subseteq U \mid \mathrm{Fl}\left(U^{\prime}, \sim U\right)\right\}=U\right)$; hence $M(X, G) \vDash \Phi_{1}^{0}$ and hence it belongs to $K^{(1)}$.

$K^{R D 2}$ does not have a natural definition; however, its subclass $K^{L C}$ is naturally defined, and it contains some interesting subclasses. Also note that if $M(X, G) \in K^{L C}$ and $G \subseteq H \subseteq H(X)$, then $M(X, H) \in K^{L C}$.

In the following corollary we list some subclasses of $K^{L C}$; these classes are faithful since $K^{L C}$ is faithful.

Corollary 3.6. The following classes are contained in $K^{L C}$, and hence they are faithful.

(a) (Whittaker [W]) $\{X \mid X$ is a Euclidean manifold $\}$.

(b) $\{M(X, G) \mid X$ is a PL-manifold and $P L(X) \subseteq G \subseteq H(X)\}$.

(c) $\left\{M(X, G) \mid\right.$ for some $k \leq \infty \quad X$ is a $C^{k}$-manifold and $C^{k}(X) \subseteq G \subseteq$ $H(X)\}$.

(d) Let $X$ be a manifold with an atlas $\left\{\varphi_{i} \mid i \in I\right\}$ and $K$ be a number such that for every $i, j \in I, \varphi_{i} \circ \varphi_{j}^{-1}$ has a Lipschitz constant less than $K$; we call such a manifold a Lipschitz manifold and it makes sense to say that $f \in H(X)$ 
is Lipschitz. We denote $L(X)=\left\{f \in H(X) \mid f\right.$ and $f^{-1}$ are Lipschitz $\}$. Then $\{M(X, G) \mid X$ is a Lipschitz manifold and $L(X) \subseteq G \subseteq H(X)\}$ is contained in $K^{L C}$ and is hence faithful.

Many variants of (a), (b), (c), and (d) which are obtained by adding structure (like volume) give rise to subclasses of $K^{L C}$.

(e) The following class is contained in $K^{L C}:\left\{X \mid X=\prod_{i \in I} X_{i}\right.$ for every $i \in I$ $X_{i}$ is a connected Euclidean manifold and $X_{i}$ is compact for all but finitely many members of $I\}$.

Definition 3.7. Let $K$ be a class of $M(X, G)$ 's. We say that $M(Y, H)$ is a $K$-manifold if for every $y \in Y$ there are $M(X, G) \in K$, an open $T \subseteq X$, and an $h: \operatorname{cl}(T) \rightarrow Y$ such that (1) $h$ is a homeomorphism between $\operatorname{cl}(T)$ and $h(\operatorname{cl}(T)) ;(2) h(\operatorname{cl}(T))$ is closed in $Y ;(3) h(T)$ is open in $Y ;(4) y \in h(T)$; and (5) for every $f \in G$ : if $\operatorname{var}(f) \subseteq T$, then $\tilde{f} \stackrel{\text { def }}{=} h f h^{-1} \cup$ Id $\uparrow(Y-h(T)) \in$ $H$. (Note that conditions (1)-(3) imply that $\tilde{f} \in H(Y)$.)

If $K$ is a class of spaces, then we say that $Y$ is a $K$-manifold if $M(Y, H(Y))$ is an $\{M(X, H(X)) \mid X \in K\}$-manifold.

Let $\operatorname{Man}(K)$ be the class of $K$-manifolds. Clearly $\operatorname{Man}(K) \subseteq \operatorname{Reg}(K)$.

Proposition 3.8. (a) $K^{L C}=\operatorname{Man}\left(K^{L C}\right)$.

(b) If $\lambda$ is an infinite cardinal, then $[0,1]^{\lambda} \in K^{L C}$. (In particular 3.6 applies to Hilbert cube manifolds and any $[0,1]^{\lambda}$-manifold, $\lambda \geq \aleph_{0}$. This strengthens [M].)

Proof. (a) is trivial. (b) follows easily from the fact that $[0,1]^{\aleph_{0}}$ is homogeneous. (See [BP].)

Remark. It is easy to construct for every separable $X$ such that $M(X) \in K^{R D 2}$ a countable $G \subseteq H(X)$ such that $M(X, G) \in K^{R D 2}$. On one hand, this means that 3.6 is stronger than [W]. On the other hand, it shows that in the presence of local compactness, one needs just a small portion of $H(X)$ in order to reconstruct $X$, and this means that local compactness can be regarded as too strong an assumption.

3.6 is too restricted also in another direction; it requires all points of $X$ to be densely conjugated. This is not the case for polyhedra or for manifolds with boundary.

\section{Normed manifolds}

Our next two goals are to drop the assumption of local compactness and to include spaces in which not every point has a somewhere dense orbit. We define (after 3.34) a class $K^{M}$; in 3.II and 3.III we prove that $K^{M}$ is faithful and in 3.IV we prove that some classes including normed manifolds, polyhedra, and manifolds with boundary are contained in $K^{M}$. We do not know how to prove that the class of locally convex topological vector spaces over $\mathbf{R}$ is faithful; the best result in this direction obtained in this work (3.VI) is the following. Let $X$ be a topological vector space over an ordered field $F . A \subseteq X$ is linearly 
bounded if for every straight line $l, A \cap l$ is bounded in $l . X$ is linearly bounded if it contains a linearly bounded nonempty open set. The class of all manifolds over locally convex linearly bounded vector spaces over an ordered field containing $\mathbf{R}$ is contained in $K^{M}$ and is thus faithful.

A subset $A$ of a Boolean algebra $B$ is dense in $B$ if for every $b \in B-\{0\}$ there is $a \in A-\{0\}$ such that $a \leq b$. Let $R \subseteq R(X)$ be dense in $R(X)$ and closed under $G$. We define $M(X, G, R) \stackrel{\text { def }}{=}\langle M(X, G), R ;\rangle, H R(X, G, R) \stackrel{\text { def }}{=}$ $\langle G, R ; \circ, \subseteq, \mathrm{Op}\rangle$, and $\operatorname{HRP}(X, G, R)=\langle H R(X, G, R), X ; \in, \mathrm{Op}\rangle$; note that $H R(X, G, V(X, G))=H V(X, G)$. The main result in this part (Corollary 3.20(a)) is that for a certain class $K$ of $M(X, G, R)$ 's $\{H R P(X, G, R) \mid$ $M(X, G, R) \in K\}$ is first-order interpretable in $\{H R(X, G, R) \mid M(X, G, R) \in$ $K\}$. In all applications $R$ will be $V(X, G)$, but this is irrelevant to our proof. Combining the fact that $\operatorname{HR}(X, G, V(X, G))$ is first-order interpre table in $G$ and that $\operatorname{HRP}(X, G, V(X, G))$ is first-order interpretable in $H R(X, G, V(X, G))$, we shall conclude that for a certain class of $M(X, G)$ 's $\langle X, V(X, G) ; \in\rangle$ is first-order interpretable in $G$ (Corollary 3.20(b)).

In what follows $M(X, G, R)$ is fixed, $T, S$ denote elements of $\tau_{X}$, and $U, V, W$ denote elements of $R$.

Definitions 3.9 and 3.11 are based on the relations and functions of $M(X, G)$ and do not use the distinguished subset $R$ of $R(X)$ mentioned in $M(X, G, R)$.

Definition 3.9. (a) $T$ is small if $|T|>1$ and for every $\varnothing \neq T_{1} \subseteq T$ there is $f \in G$ such that $f(T) \subseteq T_{1}$.

(b) $T$ is strongly small in $S$ ( $T$ is $S S$ in $S$ ) if $S \neq \varnothing$ and for every $\varnothing \neq S_{1} \subseteq S$ there is $f \in G$ such that $\operatorname{var}(f) \subseteq S$, and $f(T) \subseteq S_{1}$.

If $T$ is small, let $\operatorname{sc}(T) \stackrel{\text { def }}{=} \bigcup\left\{T^{\prime} \mid T^{\prime} \cong T\right\} . \operatorname{sc}(T)$ is called the small component of $T$. Let $S C(X, G) \stackrel{\text { def }}{=}\{\operatorname{sc}(T) \mid T$ is small in $M(X, G)\}$. Clearly every small component is open, and every two distinct small components are disjoint.

Let $S(X, G) \stackrel{\text { def }}{=} \cup\{T \mid T$ is small in $M(X, G)\}$. Our goal is to interpret $S(X, G)$ in $H R(X, G, R)$.

Proposition 3.10. (a) Let $S$ be a small component of $M(X, G)$, and suppose there is an open $T_{1} \neq \varnothing$ such that $\operatorname{cl}\left(T_{1}\right) \subseteq S$; then if $T \subseteq S$ is small, then $\operatorname{cl}(T) \subseteq S$. If in addition, $\operatorname{cl}\left(T_{1}\right)$ is contained in a small set, then $\operatorname{cl}(T)$ is contained in a small set.

(b) If $T$ is $S S$ in $S$ and $X$ is Hausdorff, then $\operatorname{cl}(T) \subseteq S$.

(c) If $T$ is small and $\varnothing \neq S \subseteq T$, then $S$ is small and $\operatorname{sc}(S)=\operatorname{sc}(T)$.

Proof. Trivial.

Definition 3.11. $T$ is excellent if (1) $T$ is small; (2) for every $S$ : if $\operatorname{cl}(S) \subseteq T$, then $S$ is $S S$ in $T$; (3) for every $x \in \operatorname{cl}(T)$ and small $S \subseteq \operatorname{sc}(T)$, there is $S^{\prime} \cong S$ such that $S^{\prime} \subseteq T$ and $x \in \operatorname{cl}\left(S^{\prime}\right)$; and (4) for every $S$, if $T \cap S=\varnothing$, then there is $T^{\prime} \cong T$ such that $T^{\prime} \cap S=\varnothing$, and $\operatorname{cl}(T)-\operatorname{cl}(S) \subseteq T^{\prime}$. 
Note that (1) is implied by the conjunction of (2) and (4). Note that an open ball is excellent in $\mathbf{R}^{n}$.

If $A \subseteq X$ let $\operatorname{bd}(A) \stackrel{\text { def }}{=} \operatorname{cl}(A)-\operatorname{int}(A)$.

Definition 3.12. Let $T$ be open; $T$ is recognizably clopen in $M(X, G)(T$ is $R C L)$ if $T$ is clopen and for every $f \in G$ such that $f(T) \cap T=\varnothing$, $f \nmid T \cup f^{-1} \uparrow f(T) \cup \operatorname{Id} \uparrow(X-T-f(T)) \in G$.

Note that if $G=H(X)$, then every clopen set is recognizably clopen.

Definition 3.13. (a) Let $S$ be a small component in $M(X, G) . S$ is excellently structured (relative to $M(X, G, R)$ if (1) for every $x \in S, T \ni x$, and small $V \subseteq S$ (recall that $V, U, W$ denote members of $R$ ), there is $V^{\prime} \cong V$ such that $x \in V^{\prime} \subseteq \operatorname{cl}\left(V^{\prime}\right) \subseteq T$; and (2) for every $x \in S$ and $T \ni x$ there are $V$ and an excellent $U$ such that $U \cap V=\varnothing, U \subseteq T$, and $\operatorname{cl}(U) \cap \operatorname{cl}(V)=\{x\}$.

(b) Let $S$ be a small component of $M(X, G) . S$ is clopenly structured (relative to $M(X, G, R)$ ) if (1) every small clopen subset of $S$ is recognizably clopen; (2) for every $x \in S, x$ has a base for its neighborhood system consisting of clopen sets which belong to $R$; and (3) for every $x \in S$ there is $V \in R$ such that $\operatorname{bd}(V)=\{x\}$.

(c) Let $K^{E C S}$ be the class of $M(X, G, R)$ 's such that $X$ is Hausdorff, and for every $S \in S C(X, G)$ either $S$ is excellently structured or $S$ is clopenly structured. (Recall that $R$ is assumed to be a dense subset of $R(X)$.) Let $K_{1}^{E C S}=\left\{H R(X, G, R) \mid M(X, G, R) \in K^{E C S}\right\}$.

Assumption 3.II.1. We consider only $\langle X, G, R\rangle$ 's such that $M(X, G, R) \in$ $K^{E C S}$.

Let $L_{H R}$ be the language of $H R(X, G, R)$.

Proposition 3.14. (a) If $S \in S C(X, G)$ and $S$ is excellently structured, then for every small $T \subseteq S, T$ is not clopen.

(b) There are first-order formulas in $L_{H R}: \varphi_{S}(U), \varphi_{S S}(U, V), \varphi_{C L}(U)$, $\varphi_{C S}(U), \varphi_{E S}(U)$, and $\varphi_{C E}(U, V)$ which in $H R(X, G, R)$ respectively mean that $U$ is small; $U$ is strongly small in $V ; U$ is small and clopen; $U$ is small and $\operatorname{sc}(U)$ is clopenly structured; $U$ is small and $\operatorname{sc}(U)$ is excellently structured; and $U$ and $V$ are small and $\operatorname{sc}(U)=\operatorname{sc}(V)$.

Proof. Easy.

Lemma 3.15. Let $\varphi_{0}(U, V) \equiv \forall W\left(\varphi_{S S}(U, W) \rightarrow W \cap V \neq \varnothing\right)$, and let $\varphi_{1}(U, V)$ be the formula that says (1) $U$ is small and $\operatorname{sc}(U)$ is excellently structured; (2) $U \cap V=\varnothing$; and (3) for every small $U_{1}$ : if $\operatorname{sc}(U)=\operatorname{sc}\left(U_{1}\right)$, then there is $U_{2} \cong U_{1}$ such that $U_{2} \subseteq U$ and $\varphi_{0}\left(U_{2}, V\right)$.

(a) If $\operatorname{cl}(U) \cap \operatorname{cl}(V) \neq \varnothing$, then $H R(X, G, R) \vDash \varphi_{0}[U, V]$.

(b) If $H R(X, G, R) \vDash \varphi_{1}[U, V]$, then $\operatorname{cl}(U) \cap \operatorname{cl}(V) \neq \varnothing$.

(c) If $U$ is excellent, $U \cap V=\varnothing$, and $\operatorname{cl}(U) \cap \mathrm{cl}(V) \neq \varnothing$, then $H R(X, G, R) \vDash$ $\varphi_{1}[U, V]$. 
Proof. (a) Let $\operatorname{cl}(U) \cap \operatorname{cl}(V) \neq \varnothing$, and suppose $U$ is $S S$ in $W$. By $3.10(\mathrm{~b})$ $\operatorname{cl}(U) \subseteq W$, hence $W \cap \operatorname{cl}(V) \neq \varnothing$, and hence $W \cap V \neq \varnothing$. This means that $H R(X, G, R) \vDash \varphi_{0}[U, V]$.

(b) Suppose $\operatorname{cl}(U) \cap \mathrm{cl}(V)=\varnothing$, and we show that $H R(X, G, R) \vDash \neg \varphi_{1}[U, V]$. Without loss of generality $U$ is small and $\operatorname{sc}(U)$ is excellently structured, so there is an excellent $U_{1}$ such that $\operatorname{sc}\left(U_{1}\right)=\operatorname{sc}(U)$. Let $U_{2} \cong U_{1}$ and $U_{2} \subseteq U$, hence $\operatorname{cl}\left(U_{2}\right) \cap \operatorname{cl}(V)=\varnothing$. By 3.11(4) there is $W \cong U_{2}$ such that $\operatorname{cl}\left(U_{2}\right) \subseteq W$ and $W \cap V=\varnothing$. Since $W$ is excellent by 3.11(2) $U_{2}$ is $S S$ in $W$. This shows that $H R(X, G, R) \vDash \neg \varphi_{0}\left[U_{2}, V\right]$. Hence there is no $U_{2} \cong U_{1}$ such that $U_{2} \subseteq U$ and $\varphi_{0}\left(U_{2}, V\right)$ holds, so $H R(X, G, R) \vDash \neg \varphi_{1}[U, V]$.

(c) Let $U$ and $V$ be as in (c); of the three requirements in $\varphi_{1}(U, V)$ the first two are trivially satisfied. To prove (3), let $U_{1}$ be small and $\operatorname{sc}\left(U_{1}\right)=\operatorname{sc}(U)$. Let $x \in \operatorname{cl}(U) \cap \operatorname{cl}(V)$. By 3.11(3) there is $U_{2} \cong U_{1}$ such that $U_{2} \subseteq U$ and $x \in \operatorname{cl}\left(U_{2}\right)$. Hence $\operatorname{cl}\left(U_{2}\right) \cap \mathrm{cl}(V) \neq \varnothing$ and so by (a) $H R(X, G, R) \vDash \varphi_{0}\left[U_{2}, V\right]$. This proves (3) in the definition of $\varphi_{1}$.

Lemma 3.16. Let

$$
\begin{aligned}
\varphi_{2}(U, V) \equiv \varphi_{1}(U, V) & \wedge\left(\forall U_{1}, U_{2}\right) \\
& \left(\left(\bigwedge_{i=1}^{2} U_{i} \subseteq U \wedge \bigwedge_{i=1}^{2} \varphi_{0}\left(U_{i}, V\right)\right) \rightarrow \varphi_{0}\left(U_{1}, U_{2}\right)\right) .
\end{aligned}
$$

(a) If $H R(X, G, R) \vDash \varphi_{2}[U, V]$, then $|\operatorname{cl}(U) \cap \operatorname{cl}(V)|=1$.

(b) If $U$ is excellent and $|\operatorname{cl}(U) \cap \mathrm{cl}(V)|=1$, then $H R(X, G, R) \vDash \varphi_{2}[U, V]$. 1 .

(c) If $H R(X, G, R) \vDash \varphi_{2}[U, V] \wedge U_{1} \subseteq U \wedge \varphi_{0}\left[U_{1}, V\right]$, then $\left|\operatorname{cl}\left(U_{1}\right) \cap \mathrm{cl}(V)\right|=$

(d) If $x \in S \in S C(X, G)$ and $S$ is excellently structured, then there are $U$ and $V$ such that $\{x\}=\operatorname{cl}(U) \cap \operatorname{cl}(V)$ and $H R(X, G, R) \vDash \varphi_{2}[U, V]$.

Proof. (a) Let $H R(X, G, R) \vDash \varphi_{2}[U, V]$. By 3.15(b) $\operatorname{cl}(U) \cap \operatorname{cl}(V) \neq \varnothing$. Suppose by contradiction that $x_{1} \neq x_{2}$ and $x_{1}, x_{2} \in \operatorname{cl}(U) \cap \operatorname{cl}(V)$. By 3.13(a)(1) and by the smallness of $U$, there is $U^{\prime}$ such that $\varnothing \neq \operatorname{cl}\left(U^{\prime}\right) \subseteq U$. By $3.10(\mathrm{a}) \operatorname{cl}(U) \subseteq \operatorname{sc}(U)$, and hence $x_{1}, x_{2} \in \operatorname{sc}(U)$. Let $T_{1}, T_{2}$ be disjoint neighborhoods of $x_{1}$ and $x_{2}$, respectively. Since $\operatorname{sc}(U)$ is excellently structured, by $3.13(\mathrm{a})(2)$ there is an excellent $W \subseteq \operatorname{sc}(U)$. By the excellence of $W$ it is small, so by $3.13(\mathrm{a})(1)$ there are $W_{1} \cong W_{2} \cong W$ such that $x_{i} \in W_{i} \subseteq \operatorname{cl}\left(W_{i}\right) \subseteq T_{i}, i=1,2$. Hence, $\operatorname{cl}\left(W_{1}\right) \cap \mathrm{cl}\left(W_{2}\right)=\varnothing$. By 3.11(4) there is $W_{3} \cong W_{1}$ such that $\mathrm{cl}\left(W_{1}\right) \subseteq W_{3}$ and $W_{3} \cap W_{2}=\varnothing$, and hence by 3.11(2) $W_{1}$ is $S S$ in $W_{3}$. Let $U_{i}=U \cap W_{i}$. Since $x_{i} \in \operatorname{cl}\left(U_{i}\right) \cap \mathrm{cl}(V)$, by 3.15(a), $H R(X, G, R) \vDash \varphi_{0}\left[U_{i}, V\right], i=1,2$. Since $W_{1}$ is $S S$ in $W_{3}$ and $U_{1} \subseteq W_{1}$, $U_{1}$ is $S S$ in $W_{3}$. But $W_{3} \cap U_{2}=\varnothing$, hence $H R(X, G, R) \not \varphi_{0}\left[U_{1}, U_{2}\right]$. This shows that $H R(X, G, R) \not \models \varphi_{2}[U, V]$, a contradiction, so (a) is proved.

(b) Let $U$ and $V$ be as in (b), and let $\{x\}=\operatorname{cl}(U) \cap \operatorname{cl}(V)$. Clearly $U \cap V=\varnothing$ and hence by $3.15(\mathrm{c}) H R(X, G, R) \vDash \varphi_{1}[U, V]$. By 3.11(4) there is $U^{\prime} \cong$ $U$ such that $U^{\prime} \cap V=\varnothing$ and $\operatorname{cl}(U)-\operatorname{cl}(V) \subseteq U^{\prime}$. Suppose $W \subseteq U$ and $H R(X, G, R) \vDash \varphi_{0}[W, V]$. Since $U^{\prime} \cap V=\varnothing, W$ is not $S S$ in $U^{\prime}$. By 3.11(2) 
$\operatorname{cl}(W) \nsubseteq U^{\prime}$. But $\operatorname{cl}(W)-\operatorname{cl}(V) \subseteq \operatorname{cl}(U)-\operatorname{cl}(V) \subseteq U^{\prime}$, hence $\operatorname{cl}(W) \cap \operatorname{cl}(V) \nsubseteq U^{\prime}$, so $x \in \operatorname{cl}(W)$. We have thus shown that if $W_{1}, W_{2} \subseteq U$ and $H R(X, G, R)$ F $\bigwedge_{i=1}^{2} \varphi_{0}\left[W_{1}, V\right]$, then $\operatorname{cl}\left(W_{1}\right) \cap \operatorname{cl}\left(W_{2}\right) \supseteq\{x\} \neq \varnothing$; by 3.15(a) it follows that $H R(X, G, R) \vDash \varphi_{0}\left[W_{1}, W_{2}\right]$. This means that $H R(X, G, R) \vDash \varphi_{2}[U, V]$, and (b) is proved.

(c) Let $U, V, U_{1}$ be as in (c). Suppose by contradiction that $\operatorname{cl}\left(U_{1}\right) \cap$ $\operatorname{cl}(V)=\varnothing$. Let $\{x\}=\operatorname{cl}(U) \cap \operatorname{cl}(V)$. Since $\operatorname{sc}(U)$ is excellently structured, by $3.13(\mathrm{a})(2)$ there is an excellent $W^{\prime}$ such that $\operatorname{sc}\left(W^{\prime}\right)=\operatorname{sc}(U)$. By 3.3(a)(1) there is $W \cong W^{\prime}$ such that $x \in W \subseteq \operatorname{cl}(W) \subseteq X-\operatorname{cl}\left(U_{1}\right)$. By 3.11(4) there is $W_{1} \cong W$ such that $W_{1} \cap U_{1}=\varnothing$ and $\operatorname{cl}(W) \subseteq W_{1}$. By 3.11(2) $W$ is $S S$ in $W_{1}$, and hence $U_{2} \stackrel{\text { def }}{=} U \cap W$ is $S S$ in $W_{1}$. These facts mean that (i) $H R(X, G, R) \vDash \neg \varphi_{0}\left[U_{2}, U_{1}\right]$. Since $x \in \operatorname{cl}\left(U_{2}\right) \cap \operatorname{cl}(V)$, by 3.15(a) (ii) $H R(X, G, R) \vDash \varphi_{0}\left[U_{2}, V\right]$. It is assumed that (iii) $H R(X, G, R) \vDash \varphi_{0}\left[U_{1}, V\right]$. But (i), (ii), and (iii) imply that $H R(X, G, R) \vDash \neg \varphi_{2}[U, V]$, a contradiction; hence (c) is proved.

(d) follows trivially from $3.13(a)(2)$ and (b).

Lemma 3.17. Let

$$
\begin{aligned}
\varphi_{3}\left(U_{1}, V_{1}, U_{2}, V_{2}\right) \equiv\left(\forall U_{1}^{\prime} \subseteq U_{1}\right)\left(\forall U_{2}^{\prime} \subseteq U_{2}\right) \\
\left(\left(\bigwedge_{i=1}^{2} \varphi_{0}\left(U_{i}^{\prime}, V_{i}\right)\right) \rightarrow \varphi_{0}\left(U_{1}^{\prime}, U_{2}^{\prime}\right)\right) .
\end{aligned}
$$

Then for every $U_{1}, V_{1}, U_{2}, V_{2}$ : if $H R(X, G, R) \vDash \bigwedge_{i=1}^{2} \varphi_{2}\left[U_{i}, V_{i}\right]$, then $H R(X, G, R) \vDash \varphi_{3}\left[U_{1}, V_{1}, U_{2}, V_{2}\right]$ iff $\operatorname{cl}\left(U_{1}\right) \cap \operatorname{cl}\left(V_{1}\right)=\operatorname{cl}\left(U_{2}\right) \cap \operatorname{cl}\left(V_{2}\right)$.

Proof. Suppose $H R(G, X, R) \vDash \bigwedge_{i=1}^{2} \varphi_{2}\left[U_{i}, V_{i}\right]$. Proof of $\Rightarrow$. Assume $H R(X, G, R) \vDash \varphi_{3}\left[U_{1}, V_{1}, U_{2}, V_{2}\right]$, and suppose by contradiction $\operatorname{cl}\left(U_{1}\right) \cap$ $\operatorname{cl}\left(V_{1}\right)=\left\{x_{1}\right\} \neq\left\{x_{2}\right\}=\operatorname{cl}\left(U_{2}\right) \cap \operatorname{cl}\left(V_{2}\right)$.

Let $W_{i}$ be excellent, $x_{i} \in W_{i}, i=1,2$, and $\operatorname{cl}\left(W_{1}\right) \cap \operatorname{cl}\left(W_{2}\right)=\varnothing$. Let $U_{i}^{\prime}=U_{i} \cap W_{i}, i=1,2$; then $H R(X, G, R) \vDash \wedge_{i=1}^{2}\left(U_{i}^{\prime} \subseteq U_{i} \wedge \varphi_{0}\left[U_{i}^{\prime}, V_{i}\right]\right) \wedge$ $\neg \varphi_{0}\left[U_{1}^{\prime}, U_{2}^{\prime}\right]$, so $H R(X, G, R) \vDash \neg \varphi_{3}\left[U_{1}, V_{1}, U_{2}, V_{2}\right]$, a contradiction.

Proof of $\Leftarrow$. Assume $\operatorname{cl}\left(U_{1}\right) \cap \operatorname{cl}\left(V_{1}\right)=\operatorname{cl}\left(U_{2}\right) \cap \operatorname{cl}\left(V_{2}\right)=\{x\}$, and $H R(X, G, R)$ $\vDash \bigwedge_{i=1}^{2} \varphi_{2}\left[U_{i}, V_{i}\right]$. Let $U_{i}^{\prime} \subseteq U_{i} i=1,2$, and $H R(X, G, R) \vDash \varphi_{0}\left[U_{i}^{\prime}, V_{i}\right]$. By $3.16(\mathrm{c}) \quad x \in \operatorname{cl}\left(U_{i}^{\prime}\right) \quad i=1,2$, and by $3.15(\mathrm{a}) H R(X, G, R) \vDash \varphi_{0}\left[U_{1}^{\prime}, U_{2}^{\prime}\right]$. So $H R(X, G, R) \vDash \varphi_{3}\left[U_{1}, V_{1}, U_{2}, V_{2}\right]$. Q.E.D.

Lemma 3.18. . Let $\varphi_{4}(U, V, W) \equiv\left(\exists W_{1}, W_{2}, U_{1}, V_{1}\right)\left(\left(W_{1}\right.\right.$ is SS in $\left.W_{2}\right) \wedge$ $\left.\left(W_{2} \subseteq W\right) \wedge\left(U_{1} \subseteq W_{1}\right) \wedge \varphi_{2}\left(U_{1}, V_{1}\right) \wedge \varphi_{3}\left(U, V, U_{1}, V_{1}\right)\right)$. Then if $H R(X, G, R)$ $\vDash \varphi_{2}[U, V]$, then for every $W: H R(X, G, R) \vDash \varphi_{4}[U, V, W]$ iff $\operatorname{cl}(U) \cap \operatorname{cl}(V) \subseteq$ $W$.

Proof. Let $H R(X, G, R) \vDash \varphi_{2}[U, V]$, and let $W \in R$.

First direction. Suppose $\operatorname{cl}(U) \cap \operatorname{cl}(V)=\{x\} \subseteq W$. Let $W_{2}$ be excellent, and $x \in W_{2} \subseteq W$. There is $W_{1}$ such that $x \in W_{1} \subseteq \operatorname{cl}\left(W_{1}\right) \subseteq W_{2}$. So $W_{1}$ is $S S$ in 
$W_{2}$. There are $V_{1}$ and an excellent $U_{1} \subseteq W_{1}$ such that $\{x\}=\operatorname{cl}\left(U_{1}\right) \cap \operatorname{cl}\left(V_{1}\right)$. Hence by 3.16(b) and $3.17 H R(X, G, R) \vDash \varphi_{2}[U, V] \wedge \varphi_{3}\left[U, V, U_{1}, V_{1}\right]$. This amounts to proving that $H R(X, G, R) \vDash \varphi_{4}[U, V, W]$.

Second direction. Suppose $H R(X, G, R) \vDash \varphi_{2}[U, V] \wedge \varphi_{4}[U, V, W]$. Let $W_{1}$, $W_{2}, U_{1}, V_{1}$ be as assured by $\varphi_{4}$. Let $\{x\}=\operatorname{cl}\left(U_{1}\right) \cap \operatorname{cl}\left(V_{1}\right)$. Clearly $\{x\}=$ $\operatorname{cl}(U) \cap \operatorname{cl}(V) . \quad x \in \operatorname{cl}\left(U_{1}\right) \subseteq \operatorname{cl}\left(W_{1}\right)$. Since $W_{1}$ is $S S$ in $W_{2} \operatorname{cl}\left(W_{1}\right) \subseteq W_{2}$. Hence $x \in \operatorname{cl}\left(U_{1}\right) \subseteq \operatorname{cl}\left(W_{1}\right) \subseteq W_{2} \subseteq W$. Q.E.D.

Let us now consider the formulas $\varphi_{2}(U, V), \varphi_{3}\left(U_{1}, V_{1}, U_{2}, V_{2}\right)$, and $\varphi_{4}(U, V, W)$. We shall use pairs $\langle U, V\rangle$ which satisfy $\varphi_{2}$ to represent all the points which belong to an excellently structured small component. By the definition of $\varphi_{2}$ and by $3.16(\mathrm{a})$ and (d) $\left\{x \mid(\exists U, V)\left(H R(X, G, R) \vDash \varphi_{2}[U, V]\right.\right.$ and $\{x\}=\operatorname{cl}(U) \cap \operatorname{cl}(V))\}=\bigcup\{S \in S C(X, G) \mid S$ is excellently structured $\}$. $\varphi_{3}\left(U_{1}, V_{1}, U_{2}, V_{2}\right)$ tells which pairs represent the same point and $\varphi_{4}(U, V, W)$ tells when the point represented by $\langle U, V\rangle$ belongs to $W$.

Now we carry out a similar procedure for the clopenly structured small components.

Lemma 3.19. Let $\varphi_{5}(U)$ be the formula which says (1) $U$ is small, and $\operatorname{sc}(U)$ is clopenly structured; (2) for every small and clopen $V$ either $U \cap V$ is small and clopen or $U \cap V=\varnothing$, or $U \sim V$ is small and clopen or $U \sim V=\varnothing$; and (3) $U$ is not small and clopen.

(a) For every $U \in R \quad H R(X, G, R) \vDash \varphi_{5}[U]$ iff $U$ is small, $\operatorname{sc}(U)$ is clopenly structured, and $|\mathrm{bd}(U)|=1$

(b) For every $x \in S \in S C(X, G)$ : if $S$ is clopenly structured, then there is $U$ such that $H R(X, G, R) \vDash \varphi_{5}[U]$ and $\operatorname{bd}(U)=\{x\}$.

(c) Let $\varphi_{6}(U, V)$ say that for every small and clopen $W: U \cap W$ is small and clopen or $U \cap W=\varnothing$ iff $V \cap W$ is small and clopen or $V \cap W=\varnothing$.

Then for every $U, V$ such that $H R(X, G, R) \vDash \varphi_{5}[U] \wedge \varphi_{5}[V]$ :

$$
H R(X, G, R, R) \vDash \varphi_{6}[U, V] \text { iff } \operatorname{bd}(U)=\operatorname{bd}(V) .
$$

(d) Let $\varphi_{7}(U, W)$ say that there is small and clopen $W_{1} \subseteq W$ such that $U \cap W_{1}$ is not empty and not small and clopen.

Then for every $U, W \in R$ such that $H R(X, G, R) \vDash \varphi_{5}[U]: H R(X, G, R) \vDash$ $\varphi_{7}[U, W]$ iff $\operatorname{bd}(U) \subseteq W$.

Proof. The proofs of (a)-(d) are very easy; however, prior to this we have to check that $\varphi_{5}, \varphi_{6}, \varphi_{7}$ can indeed be written as first-order formulas in $L_{H R}$. The formal difficulty is that even though $U \cap V$ and $U \sim V$ need not belong to $R$, we have to say about $U, V \in R$ that $U \cap V$ is small and clopen and that $U \sim V$ is small and clopen. We leave it to the reader to check that this is possible.

Let $Q \subseteq X$; we denote by $\operatorname{HRP}(X, G, R, Q)$ the submodel of $H R P(X, G)$ whose universe is $G \cup R \cup Q .(H R P(X, G)$ was defined in Definition 3.1(c).) 
Corollary 3.20. (a) Let $K_{3}^{E C S}=\{H R P(X, G, R, S(X, G)) \mid M(X, G, R) \in$ $\left.K^{E C S}\right\}$; then $K_{3}^{E C S}$ is first-order interpretable in $K_{1}^{E C S} .\left(K_{1}^{E C S}\right.$ was defined in 3.13(c).)

(b) For every $n \in \omega$ let $K^{(n)}$ be as defined just before 2.17, and let $K^{E C(n)}$ $=K^{(n)} \cap\left\{M(X, G) \mid M(X, G, V(X, G)) \in K^{E C S}\right\}$. Let $K_{0}^{E C(n)}=\{G \mid$ $\left.\exists X\left(G \subseteq H(X) \wedge M(X, G) \in K^{E C(n)}\right)\right\}$, and let

$$
K_{3}^{E C(n)}=\left\{\operatorname{HRP}(X, G, V(X, G), S(X, G)) \mid M(X, G) \in K^{E C(n)}\right\} .
$$

Then $K_{3}^{E C(n)}$ is first-order interpretable in $K_{0}^{E C(n)}$.

Proof. (a) follows easily from 3.16-3.19. (b) follows from (a) and 2.17.

Remarks. It is unnecessary to remember the exact definition of $K^{E C(n)}$. Instead one should refer to 2.32.2, where a list of concrete classes of $M(X, G)$ 's is claimed to be included in $K^{(n)}$. We shall later examine this list more carefully and check which of the $M(X, G)$ 's mentioned there happens to belong to $K^{E C(n)}$. Just in order not to leave the reader in the dark, let us mention, for the time being without proof, that if $K$ is the class of normed vector spaces over ordered fields, then the class of all regionally $K$-formed $M(X)$ 's (see Definition $2.21)$ is included in $K^{E C(2)}$. Also, Euclidean manifolds with either a differential structure or a piecewise linear structure together with the corresponding groups of homeomorphisms belong to $K^{E C(1)}$. The treatment of concrete classes of $M(X, G)$ 's appears in Theorems 3.42 and 3.43 .

2.20 (b) yields a final reconstructibility result for normed manifolds, (i.e., $K$ manifolds where $K$ is the class of normed vector spaces over ordered fields. See Definition 3.7.) If $X$ is a normed manifold, then $S(X, H(X))=X$ and $V(X, H(X))$ is an open base for $X$. Hence $H R P(X, H(X), S(X, H(X)))$, which is equal by definition to $\langle H(X), V(X, H(X)), X ; \circ, \subseteq$, Op,$\in\rangle$, determines $\left\langle X, \tau_{X}\right\rangle$.

Using the yet unproven fact that the class of normed manifolds is included in $K^{E C(2)}$, we obtain the following special case of $3.20(\mathrm{~b})$ as a corollary.

Corollary 3.21. $\{\langle H(X), V(X), X ; \circ, \subseteq$, Op,$\in\rangle \mid X$ is a normed manifold $\}$ is first-order interpretable in $\{\langle H(X), 0\rangle \mid X$ is a normed manifold $\}$; and consequently if $X, Y$ are normed manifolds and $H(X) \cong H(Y)$, then $X \cong Y$. Moreover, from the particular way that the interpretation was constructed, it follows that if $\varphi$ is an isomorphism between $H(X)$ and $H(Y)$, then there is a homeomorphism $h$ between $X$ and $Y$ such that for every $f \in H(X) \varphi(f)=h f h^{-1}$. This answers a question that was mentioned by Whittaker in [W].

III. Regionally normed spaces: polyhedra and manifolds with boundary

The abstract setting. In section II we showed how under certain assumptions $S(X, G)$ can be interpreted in $H R(X, G)$. This sufficed for $M(X, G)$ 's in which $X=S(X, G)$.

In section III we shall see how to interpret in $H R(X, G)$ some set $L(X, G) \supseteq$ $S(X, G)$ of limit points of $S(X, G)$. In a polyhedron $X$ every point $x$ which 
is an accumulation point of $\{f(x) \mid f \in H(X)\}$ belongs to $L(X, G)$. In manifolds with boundary every point which is not a boundary point of a onedimensional connected component belongs to $L(X, G)$.

We drop all previous assumptions and start a new list of ad hoc assumptions.

Assumption 3.III.1. (a) $R$ denotes an open base for $X$ consisting of regular open sets. $S$ denotes a subset of $X$.

(b) $X$ is a Hausdorff space.

(c) If $A \subseteq X, x \notin S$, and $x$ is an accumulation point of $A$, then there is a sequence $\left\{a_{i} \mid i \in \omega\right\} \subseteq A$ such that $\lim _{i \in \omega} a_{i}=x$.

We denote sequences by arrowed letters; $\vec{T}$ denotes a sequence $\left\{T_{i} \mid i \in \omega\right\}$ of open sets, $\vec{x} \stackrel{\text { def }}{=}\left\{x_{i} \mid i \in \omega\right\}, \vec{f}=\left\{f_{i} \mid i \in \omega\right\}$, and $\lim \vec{x} \stackrel{\text { def }}{=} \lim _{i \in \omega} x_{i}$.

Definition 3.22. (a) A sequence $\vec{U}$ of pairwise disjoint regular open sets such that for every $i, S \cap U_{i}$ is connected and is dense in $U_{i}$ is called a normal sequence.

(b) Let $\vec{T}$ be a sequence of open sets $\vec{x} \subseteq X$ and $\vec{f} \subseteq H(X) ; \vec{x}$ is a $\vec{T}$-sequence if for every $i \in \omega \quad x_{i} \in T_{i} ; \vec{f}$ is a $\vec{T}$-sequence if for every $i \in \omega$ $\operatorname{var}\left(f_{i}\right) \subseteq T_{i}$.

(c) $x$ is an accumulation point of $\vec{T}(x \in \operatorname{Ac}(\vec{T}))$, if $x \in \operatorname{cl}\left(\bigcup_{i \in \omega} T_{i}\right)-$ $\bigcup_{i \in \omega} \operatorname{cl}\left(T_{i}\right)$.

(d) $x=\lim _{i \in \omega} T_{i}$ if for every $\vec{T}$-sequence $\vec{x}$ we have $\lim \vec{x}=x$. We denote $\lim _{i \in \omega} T_{i}$ by $\lim \vec{T}$.

Definition 3.23. $\vec{x}$ is hereditarily self-conjugate (HSC), if for every $i \neq j$, $x_{i} \neq x_{j}$, and for every subsequence $\vec{x}^{\prime}$ of $\vec{x} \vec{x}^{\prime} \cong \vec{x}$.

Proposition 3.24. If $\vec{x}$ is HSC, then either $\vec{x}$ is convergent or $\vec{x}$ has no accumulation points.

Definition 3.25. (a) $U \in R(X)$ is transitive in $\operatorname{HRP}(X, G, R, S)$, if $S \cap U$ is dense in $U$ and for every $x, y \in U \cap S$ there is $g \in G$ such that $g(x)=y$ and $\operatorname{var}(g) \subseteq U . \vec{U}$ is transitive if for every $i \in \omega, U_{i}$ is transitive.

(b) If $\vec{f} \subseteq H(X)$ and $\left\{\operatorname{var}\left(f_{i}\right) \mid i \in \omega\right\}$ is normal then

$$
\overrightarrow{\Pi \vec{f}}=\bigcup_{i \in \omega}\left(f_{i} \uparrow \operatorname{var}\left(f_{i}\right)\right) \cup \operatorname{Id} \uparrow\left(X-\bigcup_{i \in \omega} \operatorname{var}\left(f_{i}\right)\right) .
$$

Note that $\Pi \vec{f}$ is not necessarily a homeomorphism of $X$.

(c) $\vec{U}$ has the infinite product property (IPP) if for every $\vec{U}$-sequence $\vec{f} \subseteq G$ $\Pi \vec{f}$ belongs to $G$. 
Proposition 3.26. The notions " $U$ is transitive," " $\vec{U}$ is transitive," and " $\vec{U}$ has the IPP" are expressible in terms of $\operatorname{HRP}(X, G, R, S)$.

Proposition 3.27. Let $P(\vec{U}) \equiv$ "for every subsequence $\vec{U}^{\prime}$ of $\vec{U}$, for every $\vec{U}^{\prime}$-sequence $\vec{x}^{\prime}$, and for every $x \in X$ if $\lim \vec{x}^{\prime}=x$, then $\lim \vec{U}^{\prime}=x$."

(a) If $P(\vec{U})$ holds, then $\vec{U}$ has the IPP in $H(X)$.

(b) If $\vec{U}$ is transitive and $\vec{U}$ has the IPP in $H(X)$, then $P(\vec{U})$ holds.

The following is an assumption that $G$ is closed under a certain infinitary operation of composition.

Assumption 3.III.2. If $P(\vec{U})$ holds and $\operatorname{Ac}(\vec{U}) \cap S=\varnothing$, then $\vec{U}$ has the IPP in $G$.

Definition 3.28. (a) Let $\vec{x} \subseteq S$ and $\vec{U}$ be a sequence; we say that $\vec{U}$ is a witnessing sequence for $\vec{x}$ (WS) if (i) $\vec{U}$ is normal and transitive and $\vec{x}$ is a $\vec{U}$-sequence; (ii) for every subsequence $\vec{U}^{\prime}$ of $\vec{U}$ and every $\vec{U}^{\prime}$-sequence $\vec{y}^{\prime}$ there is a subsequence $\vec{y}^{\prime \prime}$ of $\vec{y}^{\prime}$ such that $\vec{y}^{\prime \prime} \cong \vec{x}$; (iii) $\vec{U}$ does not have the IPP.

(b) Let $\operatorname{SEQ}(X, G, R, S)$ be the set of all HSC sequences from $S$ which have a WS.

Proposition 3.29. (a) If $\vec{x} \in S E Q(X, G, R, S)$, then $\vec{x}$ is convergent.

(b) $\operatorname{SEQ}(X, G, R, S)$ is definable in $\operatorname{HRP}(X, G, R, S)$.

Proof. (a) Let $\vec{x} \in \operatorname{SEQ}(X, G, R, S)$, and suppose by contradiction $\vec{x}$ is not convergent. By $3.25 \vec{x}$ has no accumulation points. Let $\vec{U}$ be a WS for $\vec{x}$. Suppose by contradiction $\operatorname{Ac}(\vec{U}) \neq \varnothing$. So there is a subsequence $\vec{U}^{\prime}$ of $\vec{U}$ and a convergent $\vec{U}^{\prime}$-sequence $\vec{y}^{\prime}$. But then if $\vec{y}^{\prime \prime}$ is a subsequence of $\vec{y}^{\prime}$, then $\vec{y}^{\prime \prime} ¥ \vec{x}$, in contradiction to (ii) of 3.28 . So $\operatorname{Ac}(\vec{U})=\varnothing$, and by Assumption 3.III $\vec{U}$ has the IPP. This contradicts (iii) of 3.28 . It follows that $\vec{x}$ is convergent.

The proof of (b) is trivial.

Now we want to express in terms of $\operatorname{HRP}(X, G, R, S)$ the fact that two sequences $\vec{x}, \vec{y} \in \operatorname{SEQ}(X, G, R, S)$ have the same limit.

Definition 3.30. (a) If $\vec{x}$ is a $\vec{U}$-sequence and $\vec{U}^{\prime}$ is a subsequence of $\vec{U}$, let $\vec{x} \uparrow \vec{U}^{\prime}$ denote the subsequence of $\vec{x}$ consisting of those $x_{i}$ 's which belong to some member of $\vec{U}^{\prime}$.

(b) For $\vec{x}, \vec{y} \in \operatorname{SEQ}(X, G, R, S)$ and a normal sequence $\vec{U}$ let $Q(\vec{x}, \vec{y}, \vec{U})$ mean (1) $\vec{U}$ is a $W S$ for $\vec{x}$; and (2) for every subsequence $\vec{U}^{\prime}$ of $\vec{U}$ and every $\vec{U}^{\prime}$-sequence $\vec{z}$, there is a subsequence $\vec{U}^{\prime \prime}$ of $\vec{U}^{\prime}$ and $g \in G$ such that $g(\vec{y})=\vec{y}$ and $g\left(\vec{x} \uparrow \vec{U}^{\prime \prime}\right)=\vec{z}^{\prime} \uparrow \vec{U}^{\prime \prime}$. 
Lemma 3.31. If $\vec{x}, \vec{y}$, and $\vec{U}$ are as in $3.30(\mathrm{~b})$ and $Q(\vec{x}, \vec{y}, \vec{U})$ holds, then $\lim \vec{x} \neq \lim \vec{y}$.

Proof. Assume that $Q(\vec{x}, \vec{y}, \vec{U})$ holds. If for every subsequence $\vec{U}^{\prime}$, of $\vec{U}$ and a $\vec{U}^{\prime}$-sequence $\vec{z}^{\prime} x \stackrel{\text { def }}{=} \lim \vec{x}$ is a limit point of $\vec{z}^{\prime}$, then $\lim \vec{U}=x$. But then $P(\vec{U})$ from 3.27 holds, and by Assumption 3.II.2 $\vec{U}$ has the IPP, contradicting the fact that $\vec{U}$ is a WS for $\vec{x}$. Hence there are a subsequence $\vec{U}^{\prime}$ of $\vec{U}$ and a $\vec{U}^{\prime}$-sequence $\vec{z}^{\prime}$ such that either $\lim \vec{z}^{\prime}$ exists and $\lim \vec{z}^{\prime} \neq x$ or $\vec{z}^{\prime}$ has no accumulation points. But since $\vec{U}$ is a WS for $\vec{x}, \vec{x} \cong \vec{z}^{\prime \prime}$ for some subsequence $\vec{z}^{\prime \prime}$ of $\vec{z}$; hence $\lim \vec{z}^{\prime} \stackrel{\text { def }}{=} z^{\prime}$ exists. Let $\vec{U}^{\prime \prime}$ and $g$ be as assured for $\vec{z}^{\prime}$ by $Q(\vec{x}, \vec{y}, \vec{U})$, hence $g(x)=z^{\prime} \neq x$ and $g(\lim \vec{y})=\lim \vec{y}$, hence $\lim \vec{x} \neq \lim \vec{y}$. Q.E.D.

Assumption 3.III.3. If $\vec{x}, \vec{y} \in \operatorname{SEQ}(X, G, R, S)$ and $\lim \vec{x} \neq \lim \vec{y}$, then $\exists \vec{U} Q(\vec{x}, \vec{y}, \vec{U})$.

Corollary 3.32. (a) $\exists \vec{U} Q(\vec{x}, \vec{y}, \vec{U})$ is expressible in $\operatorname{HRP}(X, G, R, S)$.

(b) $(\lim \vec{x}=\lim \vec{y}) \equiv \neg \exists \vec{U} Q(\vec{x}, \vec{y}, \vec{U})$, hence the fact that $\lim \vec{x}=\lim \vec{y}$ for $\vec{x}, \vec{y} \in \operatorname{SEQ}(X, G, R, S)$ is expressible (by a second-order formula) in $H R P(X, G, R, S)$.

Proof. Trivial.

Next we want to express in $\operatorname{HRP}(X, G, R, S)$ the fact that $\lim \vec{x} \in U$.

Let $L=L(X, G, R, S) \stackrel{\text { def }}{=} S \cup\{\lim \vec{x} \mid \vec{x} \in \operatorname{SEQ}(X, G, R, S)\}$.

Assumption 3.III.4. For every $x \in L$ and open $T \ni x$ there is $g \in G$ such that $g(x) \neq x$ and $\operatorname{var}(g) \subseteq T$.

Proposition 3.33. Let $\vec{x} \in \operatorname{SEQ}(X, G, R, S)$ and $U \in R$; then $\lim \vec{x} \in U$ iff there is $g \in G$ such that $\operatorname{var}(g) \subseteq U$ and $\lim \vec{x} \neq \lim g(\vec{x})$.

Proof. Trivial.

Corollary 3.34. $H R P(X, G, R, L(X, G, R, S))$ is second-order interpretable in $H R P(X, G, R, S)$.

Let us summarize for the reader what we have achieved in $\S \S 2$, 3.II, and 3.III. Let $K^{M}$ be the class of all $M(X, G)$ 's that have the following properties: (1) $M(X, G) \in K^{0}$ (see definition before 2.14); (2) $M(X, G, R(X)) \in K^{E C S}$ (see Definition 3.13); (3) $H R P(X, G, R(X), S(X, G)$ ) satisfies Assumptions 3.III.1-3.III.4 (see the notation preceding 3.10 for $S(X, G)$ and the notation preceding 3.20 for $H R P(X, G, R, S))$; and (4) $L(X, G, R(X), S(X, G))=X$.

Let $K_{0}^{M}=\left\{G \mid \exists X\left(M(X, G) \in K^{M}\right)\right\}$. 
Theorem 3.35. $K^{M}$ is interpretable in $K_{0}^{M}$.

Proof. Combine the results of 2.14, 3.II, and 3.III.

Remarks. (a) In the formulation of 3.35 we did not try to obtain a first-order interpretation. Indeed by slightly modifying the definition of $K^{M}$ and some extra work we can obtain a first-order interpretation of $K^{M}$ in $K_{0}^{M}$. This will be done in [R3], where a first-order interpretation is essential.

(b) $K^{M}$ is of course a very artificial class. The only reason for defining $K^{M}$ is to obtain a better presentation. Indeed we are interested in the class which includes (1) normed manifolds, (2) manifolds with boundary, (3) polyhedra, and (4) smooth and $P L$-manifolds with their corresponding groups. So it remains to show that $K^{M}$ includes these classes.

(c) Assumption 3.III.2 is very restrictive. It unfortunately excludes the groups of smooth $(P L)$ homeomorphisms of a smooth $(P L)$ manifold with boundary. However, it is not too difficult to modify the assumptions in such a way that these cases will be included in our results. Ling's work [Lg] covers these cases.

IV. Regionally normed spaces (continued)

Our next goals are to show that every regionally normed space satisfies the assumptions of 3.II and that every Euclidean manifold with boundary and every polyhedron satisfies the assumptions of 3.III.

Let $Y$ denote a normed vector space over an ordered field $F$. Let $B_{Y}=$ $\{x \in Y \mid\|x\|<1\}, \bar{B}_{Y}=\mathrm{cl}\left(B_{Y}\right)$, and $S_{Y}=\operatorname{bd}\left(\bar{B}_{Y}\right)$.

If $A, B \subseteq Y$ and $\lambda \in F$, let $A+B=\{a+b \mid a \in A, b \in B\}$ and $\lambda A=\{\lambda a \mid$ $a \in A\}$.

Definition 3.36. (a) Let $h: \bar{B}_{Y} \rightarrow X, h$ is cellular, if $h$ is a homeomorphism into $X, h\left(\bar{B}_{Y}\right)$ is closed and $h\left(B_{Y}\right)$ is open. $h\left(B_{Y}\right)$ is called a cell, $h\left(\frac{1}{2} \cdot B_{Y}\right)$ is called a half cell.

(b) $N(X)=\bigcup\{T \mid T$ is a cell $\}$.

(c) $X$ is regionally normed if $N(X)$ is dense in $X$.

We drop all previous assumptions.

Assumption 3.IV.1. $X$ is regionally normed.

Lemma 3.37. (a) If $V \subseteq X$ is a cell, then $\mathrm{Fl}(V ; \sim V)$ holds in $M(X)$.

(b) $M(X) \in K^{(2)}$. (See the definition of $K^{(2)}$ after 2.16.)

Proof. Easy.

Notational Convention. Whenever $G=H(X)$ we omit the mention of $G$ in our notation, so $V(X)$ denotes $V(X, H(X)), S(X)$ denotes $S(X, H(X))$, etc. Whenever $R=V(X, G)$ we omit its mention in our notation, so $M(X, G)$ denotes $M(X, G, V(X, G))$ and $M(X)$ denotes $M(X, H(X), V(X))$ etc. Note that this convention causes ambiguity since now $M(X), M(X, G)$ have two meanings; however, this ambiguity is inessential since $M(X, G, V(X, G))$ is first order interpretable in $M(X, G)$. 
Lemma 3.38. (a) Every half cell is small in $M(X)$, and $S(X)=N(X)$.

(b) Let $h: \bar{B}_{Y} \rightarrow X$ be cellular and $Y$ be a vector space over a field $F$ which is not isomorphic to $\mathbf{R}$; then $U \stackrel{\text { def }}{=} \operatorname{sc}\left(h\left(\frac{1}{2} \cdot B_{Y}\right)\right.$ ) is clopenly structured (see Definition 3.13).

(c) If $h: \bar{B}_{Y} \rightarrow X$ is cellular and $Y$ is a vector space over $\mathbf{R}$, then $h\left(\frac{1}{2} \cdot B_{Y}\right)$ is excellent.

(d) If $h$ and $Y$ are as in (c), then $\operatorname{sc}\left(h\left(\frac{1}{2} \cdot B_{Y}\right)\right)$ is excellently structured.

(e) $M(X) \in K^{E C S}$.

Proof. (a) is trivial. (b) The only less trivial part of (b) is that for every $x \in U$ there is $V \in V(X)$ such that $\operatorname{bd}(V)=\{x\}$. If $Y$ is 1 -dimensional then its topology is the order topology of $F$ and it is easy to find $V$ as required.

Suppose $\operatorname{dim}(Y)>1$. It is sufficient to show that there is $V \subseteq B_{Y}$ such that $\operatorname{bd}(V)=\{0\}$, and $V \in V(Y)$. Let $\left\{\lambda_{n} \mid n \in \omega\right\}$ be an increasing sequence without a supremum of elements of $F$, and each $\lambda_{n}<1$. Let $B=\bigcup_{n \in \omega} \lambda_{n} B_{Y}$. Clearly, $B$ is clopen. Let $x_{0} \in S_{Y}$, and let $V=B \cap\{\lambda x \mid \lambda \in F,\|x\|=1$, and $\left.x \in x_{0}+B\right\}$. It is easy to see that $\operatorname{bd}(V)=\{0\}$ and $V \in V(Y)$. So (b) is proved.

(e) follows from (b) and (d).

The proof of (c) and (d) is less trivial, and we break it into several subclaims.

Lemma 3.39. Let $Y$ be a normed vector space over $\mathbf{R}$. Then:

(a) $B_{Y} \in V(Y)$.

(b) For every $0<\alpha<\beta<1$ there is $h \in H(Y)$ such that $\operatorname{var}(h) \subseteq B_{Y}$ and $h\left(\beta B_{Y}\right)=\alpha B_{Y}$.

(c) For every $0<\alpha<1, v \in B_{Y}$, and $0<\beta<1-\|v\|$, there is $h \in H(Y)$ such that $\operatorname{var}(h) \subseteq B_{Y}, h(0)=v$, and $h\left(\alpha B_{Y}\right)=v+\beta B_{Y}$.

(d) For every closed $F \subseteq B_{Y}$ there is $h \in H(Y)$ such that $\operatorname{var}(h) \subseteq B_{Y}$ and $h(F) \subseteq \frac{1}{2} \cdot B_{Y}$.

(e) For every closed $F \subseteq B_{Y}$ and every nonempty open set $U \subseteq B_{Y}$, there is $h \in H(Y)$ such that $\operatorname{var}(h) \subseteq B_{Y}$ and $h(F) \subseteq U$.

(f) For every ninempty closed $F \subseteq B_{Y}$ and $x \in S_{Y}$, there is $h \in H(Y)$ such that $\operatorname{var}(h) \subseteq 2 B_{Y}, h(F) \subseteq \bar{B}_{Y}$, and $h(F) \cap S_{Y}=\{x\}$.

(g) For every closed $F$ such that $F \cap B_{Y}=\varnothing$, there is $h \in H(Y)$ such that $\operatorname{var}(h) \subseteq 2 B_{Y}, \bar{B}_{Y}-F \subseteq h\left(B_{Y}\right)$, and $h\left(B_{Y}\right) \cap F=\varnothing$.

Proof. (a) and (b) are trivial.

(c) By (b) we can assume that $\alpha=\beta$. Let $g:[0, \infty) \rightarrow[0,1]$ be defined as follows:

$$
g(t)= \begin{cases}1, & 0 \leq t \leq \beta \\ \frac{t-1}{\beta-1}, & \beta \leq t \leq 1, \\ 0, & 1 \leq t .\end{cases}
$$

Let $h(x)=x+g(\|x\|) \cdot v$. It is easy to check that $h$ is as desired. 
Let $d(A, B)$ denote the distance between the subsets $A$ and $B$ of $Y$; $d(x, A) \stackrel{\text { def }}{=} d(\{x\}, A)$.

(d) Let $f:[0,1) \times[0,1] \rightarrow[0,1]$ be a continuous function such that for every $s \in[0,1):(1) f_{s}(t) \stackrel{\text { def }}{=} f(s, t)$ is a $1-1$ order-preserving function from $[0,1]$ onto $[0,1] ;(2) f_{s}(s) \leq \frac{1}{2}$; and (3) for every $t \in[0,1] f_{s}(t) \leq t$. Let $u: \bar{B}_{Y}-\{0\} \rightarrow[0,1)$ be defined as follows. $u(x)=\max (1-d(x /\|x\|, F), 0)$, and let

$$
h(x)= \begin{cases}0, & x=0, \\ f(u(x),\|x\|) \cdot x /\|x\|, & x \in \bar{B}_{Y}-\{0\}, \\ x, & x \in Y-B_{Y} .\end{cases}
$$

It is easy to check that $h \in H(Y)$. Let $x \in F$; we show that $\|h(x)\| \leq \frac{1}{2}$. If $x=0$ then $h(x)=0$. Suppose $x \neq 0$, then $0<d(x /\|x\|, F) \leq 1-\|x\|<1$ denote $s=d(x /\|x\|, F)$, so $h(x)=f(1-s,\|x\|) \cdot x /\|x\|$, but $s<1-\|x\|$, hence $\|x\| \leq 1-s$, so $f(1-s,\|x\|) \leq f(1-s, 1-s) \leq \frac{1}{2}$, and thus $\|h(x)\|=$ $f(1-s,\|x\|) \leq \frac{1}{2}$. So (d) is proved.

(e) This is a corollary of (c) and (d).

(f) Let $\varnothing \neq F \subseteq B_{Y}$. We define $f: S_{Y} \rightarrow[0,1]$.

$$
f(x)=\max \left(\{\lambda \mid \lambda x \in F\} \cup\left\{\frac{1}{2}\right\}\right) .
$$

Let us first prove that for every $x \in S_{Y}$ and for every $\varepsilon>0$, there is a neighborhood $U$ of $x$ such that for every $y \in S_{Y} \cap U, f(y) \leq f(x)+\varepsilon$. Suppose by contradiction such a $U$ does not exist. Hence there is a sequence $\left\{x_{n} \mid n \in \omega\right\} \subseteq S_{Y}$ such that $\lim _{n \rightarrow \infty} x_{n}=x$, but for every $n \in \omega f\left(x_{n}\right)>$ $f(x)+\varepsilon$. For every $n \in \omega$ let $\lambda_{n}>f(x)+\varepsilon$ be such that $\lambda_{n} x_{n} \in F$. Without loss of generality $\lim _{n \rightarrow \infty} \lambda_{n}=\lambda$. So $\lim _{n \rightarrow \infty} \lambda_{n} x_{n}=\lambda x$. But then $\lambda x \in F$, and $\lambda>f(x)$, a contradiction. Let $x_{0} \in S_{Y}$ and we are now seeking $h$ as required. By (c) w.l.o.g. $f\left(x_{0}\right)>\frac{1}{2}$. Let $\alpha<1$ be such that $\operatorname{Sup}(\{f(x) \mid$ $\left.\left.x \in x_{0}+\alpha \bar{B}_{Y}\right\}\right)<\left(1+f\left(x_{0}\right)\right) / 2$. Let $g:[0,2] \rightarrow\left[f\left(x_{0}\right), 1\right]$ be a continuous function such that $g(0)=f\left(x_{0}\right)$, for every $s \geq \alpha g(s)=1$, and for every $s \in(0, \alpha] g(s)>\operatorname{Sup}\left(\left\{f(x) \mid x \in x_{0}+s \bar{B}_{Y}\right\}\right)$. Let $g_{1}:\left(\frac{1}{2}, 1\right] \times(0, \infty)$ be defined as follows:

$$
g_{1}(s, t)= \begin{cases}t, & 0<t \leq \frac{1}{2} \text { or } 2 \leq t, \\ \frac{1}{2 s-1} t+\frac{s-1}{2 s-1}, & \frac{1}{2} \leq t \leq s \\ \frac{1}{2-s} t+\frac{2-2 s}{2-s}, & s \leq t \leq 2 .\end{cases}
$$

The reader can check the continuity of $g_{1}$ by noticing that for every $s \in$ $\left(\frac{1}{2}, 1\right]: g_{s}(t) \stackrel{\text { def }}{=} g_{1}(s, t)$ is the identity on $\left(0, \frac{1}{2}\right] \cup[2, \infty)$, it moves linearly $\left[\frac{1}{2}, s\right]$ onto $\left[\frac{1}{2}, 1\right]$, and it moves linearly $[s, 2]$ onto $[1,2]$. Let $h: Y \rightarrow Y$ be defined as follows:

$$
h(x)= \begin{cases}0, & x=0, \\ \left.g_{1}\left(g\left(\left\|\frac{x}{\|x\|}-x_{0}\right\|\right),\|x\|\right)\right) \cdot \frac{x}{\|x\|}, & x \neq 0 .\end{cases}
$$


Since for every $s, g_{s}$ is a $1-1$ mapping from $(0, \infty)$ onto $(0, \infty), h(x)$ is $1-1$ and onto. Since $g_{1}, g,\|x\|$ are continuous and $1 /\|x\|$ is continuous in $Y-\{0\}, h$ is continuous. Since $h \uparrow \frac{1}{2} \cdot \bar{B}_{Y}=\mathrm{Id}, h^{-1}$ is continuous at 0 . Let $g_{2}(s, t)$ be defined by the following equation: $g_{2}(s, t)=u$ iff $g_{1}(s, u)=t$. $g_{2}$ is continuous for the same reason that $g_{1}$ was. Let $x \neq 0$; then $h^{-1}(x)=$ $g_{2}\left(g\left(\|x /\| x\left\|-x_{0}\right\|,\|x\|\right) \cdot x /\|x\|\right.$, so $h^{-1}$ is continuous, hence $h \in H(X)$. $f\left(x_{0}\right) \cdot x_{0} \in F$; we now show that $h\left(f\left(x_{0}\right) \cdot x_{0}\right)=x_{0}$.

$h\left(f\left(x_{0}\right) \cdot x_{0}\right)=g_{1}\left(g\left(\left\|x_{0}-x_{0}\right\|\right), f\left(x_{0}\right)\right) \cdot x_{0}=g_{1}\left(f\left(x_{0}\right), f\left(x_{0}\right)\right) \cdot x_{0}=1 \cdot x_{0}=x_{0}$.

We now show that $h(F)-\left\{x_{0}\right\} \subseteq B_{Y}$. Let $x \in F-\left\{f\left(x_{0}\right) \cdot x_{0}\right\}-\{0\}$. $h(x)=g_{1}\left(g\left(\|x /\| x\left\|-x_{0}\right\|\right),\|x\|\right) \cdot x /\|x\|$. If $x /\|x\|=x_{0}$, then $\|x\|<f\left(x_{0}\right)$, so $h(x)=g_{1}\left(f\left(x_{0}\right),\|x\|\right) \cdot x_{0}$, but $g_{1}\left(f\left(x_{0}\right),\|x\|\right)<g_{1}\left(f\left(x_{0}\right), f\left(x_{0}\right)\right)<1$ so $\|h(x)\|<\left\|x_{0}\right\|=1$. If $x /\|x\| \neq x_{0}$, let $u=g\left(\|x /\| x\left\|-x_{0}\right\|\right)$, so $u>$ $\operatorname{Sup}\left(\left\{f(y) \mid y \in x_{0}+\|x /\| x\left\|-x_{0}\right\| \cdot \bar{B}_{Y}\right\}\right)$; but $x /\|x\| \in x_{0}+\|x /\| x\left\|-x_{0}\right\| \cdot \bar{B}_{Y}$, hence

$$
u>f(x /\|x\|) \geq \max (\{\lambda \mid \lambda \cdot x /\|x\| \in F\} \cup\{0\})>\|x\| .
$$

We thus can conclude that $\|h(x)\|=\left\|g_{1}(u,\|x\|) \cdot x /\right\| x\|\|=g_{1}(u,\|x\|)<$ $g_{1}(u, u)=1$, and hence $h(x) \in B_{Y}$, and (f) is proved.

(g) Let $F$ be as in (g). W.l.o.g. $F \neq \varnothing$. Let $f:[1,2] \times[0, \infty] \rightarrow[0, \infty]$ be defined as follows:

$$
f(s, t)= \begin{cases}s t, & 0 \leq t \leq 1 \\ (2-s) t+2 s-2, & 1 \leq t \leq 2 \\ t, & 2 \leq t\end{cases}
$$

So $f(s, t)$ moves linearly $[0,1]$ onto $[0, s],[1,2]$ onto $[s, 2]$, and $[2, \infty]$ onto $[2, \infty)$. Let

$$
h(x)= \begin{cases}0, & x=0 \\ f\left(\min \left(1+d\left(\frac{x}{\|x\|}, F\right), 1 \frac{1}{2}\right),\|x\|\right) \cdot \frac{x}{\|x\|}, & x \neq 0 .\end{cases}
$$

Clearly $h$ is as desired.

Proof of 3.38 (c) and (d). (c) Let $h$ and $Y$ be as in (c), and let $V=h\left(\frac{1}{2} \cdot B_{Y}\right)$. We show that $V$ has the four properties of excellent sets as defined in 3.11. (1) $V$ is small by 3.38(a). (2) follows from 3.39(e). (3) follows from 3.39(f). (4) follows from $3.39(\mathrm{~g})$.

(d) Let $h, Y$ be as in (d), and let $S=\operatorname{sc}\left(h\left(\frac{1}{2} \cdot B_{Y}\right)\right)$. We have to show that $S$ satisfies conditions (1) and (2) in Definition 3.13(a). Only condition (2) is nontrivial. Let $x \in S$, and let $T \ni x$ be open. Let $W \subseteq T$ be a cell containing $x$. Hence it is easy to see that there is a half cell $V_{1} \subseteq W$ such that $x \in \operatorname{bd}\left(V_{1}\right)$. Let $F \subseteq V_{1}$ be the closure of a half cell $U_{1}$. By 3.39(f) there is $g \in H(X)$ such that $g\left(U_{1}\right) \subseteq V_{1}$ and $\operatorname{bd}\left(V_{1}\right) \cap g(F)=\{x\}$, hence $g\left(U_{1}\right)$ is excellent, $g\left(U_{1}\right) \cap \sim V_{1}=\varnothing$ and $\operatorname{cl}\left(g\left(U_{1}\right)\right) \cap \operatorname{cl}\left(\sim V_{1}\right)=\{x\} . V \stackrel{\text { def }}{=} \sim V_{1}$ and $U \stackrel{\text { def }}{=} g\left(U_{1}\right)$ are as required in $3.3(\mathrm{a})(2)$, so $S$ is excellently structured. This completes the proof of $3.38(\mathrm{~d})$. 
Definition 3.40. $X$ is a normed manifold if $N(X)=X$.

Lemmas 3.37(b), 3.38(a), and 3.38(e) were the facts that remained unproved when we mentioned Corollary 3.21. At this point 3.21 is already fully proved.

Theorem 3.41. (a) Let $X$ be a $k$ times differentiable manifold $k \leq \infty$ and $G$ be a subgroup of $H(X)$ containing the group $C^{k}(X)$ of $k$ times continuously differentiable homeomorphisms; then $M(X, G) \in K^{E C(2)}$.

(b) Let $X$ be a PL-manifold and $G$ be a subgroup of $H(X)$ containing all piecewise linear homeomorphisms; then $M(X, G) \in K^{E C(2)}$.

Proof. (a) is not trivial for $k=\infty$, but the needed facts are well known.

(b) is not trivial, but the only nontrivial fact is well known; see [Gm].

Definition 3.42. (a) $X$ is a manifold with boundary if $X$ is Hausdorff and for every $x \in X$ there is $n \geq 0$ and an open neighborhood of $x$ which is homeomorphic either to $\mathbf{R}^{n}$ or to $\mathbf{R}^{n} \times[0,1)$.

(b) We define a simplicial complex as in [Sp, p. 108]. If $K$ is a simplicial complex we again use the notation of [Sp]: $|K|_{d}$ denotes $|K|$ with its metric topology, and $|K|$ denotes $|K|$ together with its coherent topology. A simplicial complex $K$ is locally finite dimensional if $K$ does not contain a strictly increasing infinite chain of simplexes. A space is called a polyhedron if it is homeomorphic to either $|K|$ or $|K|_{d}$, where $K$ is a locally finite-dimensional simplicial complex. We can enlarge the class in question by a little bit. We call $X$ a long polyhedron if $X$ has an open cover consisting of polyhedra (in the relative topology).

Lemma 3.42. Let $X$ be a manifold with boundary or a long polyhedron. Let $R=V(X)$ and $S=S(X)$; we denote $L(X, H(X), V(X), S(X))$ by $L(X)$. Then (a) $L(X)=\{x \mid x$ is an accumulation point of $\{g(x) \mid g \in H(X)\}\}$; and (b) $X, H(X), V(X), S(X)$ satisfy Assumptions 3.III.1-4. Recall that $S(X)=\bigcup\{S \mid S$ is a small open set $\}$ and $V(X)=\{\operatorname{var}(f) \mid f \in H(X)\}$.

Proof. There are several types of spaces that have to be dealt with. We deal only with the case of polyhedra with their metric topology; the other cases are in fact simpler.

Let $K=\left\langle V_{K}, \mathscr{F}_{K}\right\rangle$ be a simplicial complex. Let $Y_{K}$ be a vector space over $\mathbf{R}$ with Hamel basis $V_{K}$ and let $X_{K}=\left\{x \in Y_{K} \mid\right.$ for some $F \in \mathscr{F}_{K}$ $x$ is in the convex hull of $F$. We abbreviate $V_{K}, \mathscr{F}_{K}$, and $X_{K}$ by $V$, $\mathscr{F}$, and $X$ respectively. If $x=\sum_{v \in V} \lambda_{v} v, y=\sum_{v \in V} \mu_{v} v$ belong to $X$ let $\left.d(x, y)=\sum_{v \in V}\left(\lambda_{v}-\mu_{v}\right)^{2}\right)^{1 / 2}$. For $F \in \mathscr{F}$ let $\operatorname{ch}(F)$ be the convex hull of $F$ in $X$. For $x=\sum_{v \in V} \lambda_{v} v$, let $F_{x}=\left\{v \mid \lambda_{v} \neq 0\right\}$. Let $\mathscr{F}^{M}=\{F \in \mathscr{F} \mid$ $\left.\neg\left(\exists F^{\prime} \in \mathscr{F}\right)\left(F \varsubsetneqq F^{\prime}\right)\right\}$. Let $S^{\prime}=\left\{x \mid F_{x} \in \mathscr{F}^{M}\right\}$. Clearly $S^{\prime} \subseteq S(X)$.

It is easy to check that Assumption 3.III.1 holds. Assumption 3.III.2 follows from $3.27(\mathrm{a})$.

Let $\operatorname{SEQ}(X)$ denote $\operatorname{SEQ}(X, H(X), V(X), S(X))$, and $L^{\prime}(X)=\{x \in X \mid x$ is an accumulation point of $\{h(x) \mid h \in H(X)\}\}$. We show that $L^{\prime}(X)=L(X)$. 
Suppose $x \notin L^{\prime}(X)$ and suppose by contradiction $x \in L(X)$. Clearly $x \notin S(X)$, hence there is $\vec{x} \in \operatorname{SEQ}(X)$ such that $x=\lim \vec{x}$. Let $\vec{U}$ be a WS for $\vec{x}$. Hence for every $y \in \operatorname{Ac}(\vec{U}) \quad y \cong x$. Hence $x$ is an isolated point of $\operatorname{Ac}(\vec{U})$. We show that $\operatorname{Ac}(\vec{U})$ is connected and $|\operatorname{Ac}(\vec{U})|>1$. Since $\vec{U}$ does not have the IPP, and since for every subsequence $\vec{U}^{\prime}$ of $\vec{U}$ and $\vec{U}^{\prime}$-sequence $\vec{y}^{\prime}, \quad \vec{y}^{\prime}$ has a convergent subsequence by $2.27(\mathrm{a})|\operatorname{Ac}(\vec{U})|>1$. Suppose by contradiction $\operatorname{Ac}(\vec{U})$ is not connected; then there are open disjoint $V$ and $W$ such that $V \cap \operatorname{Ac}(\vec{U}), W \cap \operatorname{Ac}(\vec{U}) \neq \varnothing$, and $V \cup W \supseteq \operatorname{Ac}(\vec{U})$ (this is also true in the case of the coherent topology). Suppose $x \in V$, hence for all but finitely many $i$ 's $V \cap U_{i} \neq \varnothing$. Similarly $W \cap U_{i} \neq \varnothing$ for infinitely many $i$ 's. Let $\vec{U}^{\prime}$ be a subsequence of $\vec{U}$ consisting of those $U_{i}$ 's that intersect both $V$ and $W$. Since each $U_{i}$ is connected, for every member $U_{i}$ of $\vec{U}^{\prime} \quad V \cup W \nsupseteq U_{i}$. Let $\vec{y}^{\prime}$ be a $\vec{U}^{\prime}$ sequence such that $y_{i}^{\prime} \in U_{i}-V-W$. If $y^{\prime}$ is a limit point of $\vec{y}^{\prime}$, then $y^{\prime} \notin V \cup W$ and hence $y^{\prime} \notin \operatorname{Ac}(\vec{U})$, a contradiction. Hence $\operatorname{Ac}(\vec{U})$ is connected. We have thus proved that $x$ is not isolated in $\operatorname{Ac}(\vec{U})$, hence $x \in L^{\prime}(X)$, a contradiction.

Suppose $x \in L^{\prime}(X)$, and we show that $x \in L(X)$. Since $V$ is discrete in $X$ and since $x \in \operatorname{Ac}(\{h(x) \mid h \in H(X)\})$, there is $x^{\prime} \in X-V$ such that $x^{\prime} \cong x$, and hence w.l.o.g. $x \notin V$. If $F_{x} \in \mathscr{F}^{M}$ then $x \in S^{\prime} \subseteq S(X)$, hence $x \in L(X)$. Suppose that $F_{x} \notin \mathscr{F}^{M}$. Let $F_{x} \subset F \in \mathscr{F}^{M}$. For $G=$ $\left\{u_{0}, \ldots, u_{l-1}\right\} \in \mathscr{F}$, let $\operatorname{ch}(G)$ denote the convex hull of $G$, and $\operatorname{rbd}(G) \stackrel{\text { def }}{=}$ $\left\{\sum_{i<l} \lambda_{i} u_{i} \mid \sum_{i<l} \lambda_{i}=1\right.$, for every $i<l \quad \lambda_{i} \geq 0$ and for some $\left.i<l \quad \lambda_{i}=0\right\}$. Let $K=\left\{y \in \operatorname{ch}\left(F_{x}\right) \mid d(x, y) \leq \frac{1}{2} d\left(x, \operatorname{rbd}\left(F_{x}\right)\right)\right\}$. $K$ is convex. There is an open convex set $U \subseteq \operatorname{ch}(F)$ such that $\operatorname{cl}(U) \cap \operatorname{rbd}(F)=K$. Let $x_{0} \in U$, for $i>0$ let $x_{i}=(1 / i) x_{0}+(1-1 / i) x$, and let $\vec{x}=\left\{x_{i} \mid i \in \omega\right\}$. Clearly $\lim \vec{x}=x, d_{i} \stackrel{\text { def }}{=} d\left(x_{i}, K\right)$ is strictly decreasing, and for every $i \in \omega x_{i} \in U$. We wish to show that $\vec{x} \in \operatorname{SEQ}(X) . \vec{x} \subseteq S^{\prime} \subseteq S(X)$, and it is trivial that $\vec{x}$ is HSC. Let $U_{i}=\left\{y \in U \mid d_{i}-\left(d_{i}-d_{i+1}\right) / 3<d(y, K)<d_{i}+\left(d_{i-1}-d_{i}\right) / 3\right\}$ and $\vec{U}=\left\{U_{i} \mid i \in \omega\right\} . x_{i} \in U_{i}$, hence $\vec{x}$ is a $\vec{U}$ sequence. Every $U_{i}$ is convex, so $U_{i}$ is connected and transitive; and since $U_{i} \subseteq S(X)$, this means that $\vec{U}$ is normal and transitive. It is now easy to see that $\vec{U}$ is a WS for $\vec{x}$, hence $\vec{x} \in \operatorname{SEQ}(X)$.

By a very similar construction it can be shown that if $\vec{x}, \vec{y}$ are sequences whose members are in $S(X)$ and such that $\lim \vec{x} \in L^{\prime}(X)$ and $\vec{y}$ has no subsequence converging to $\lim \vec{x}$, then there is $\vec{U}$ such that $Q(\vec{x}, \vec{y}, \vec{U})$ holds. This shows that Assumption 3.III.3 holds, and the lemma is proved. 
Corollary 3.43. Let $K=\{X \mid X$ is a normed manifold or $X$ is a manifold with boundary and $X=L(X)$ or $X$ is a long polyhedron and $X=L(X)\}$; then if $X_{1}, X_{2} \in K$, and $\varphi: H\left(X_{1}\right) \stackrel{\text { onto }}{\rightarrow} H\left(X_{2}\right)$ is an isomorphism, then there is a homeomorphism $h: X_{1} \stackrel{\text { onto }}{\rightarrow} X_{2}$ such that for every $f \in H\left(X_{1}\right): \varphi(f)=h \mathrm{fh}^{-1}$.

Proof. Combine the results of 3.20, 3.30(b), 3.34, 3.35, 3.38(e), and 3.42.

Question 5. Generalize 3.43 to complexes which are not locally finite dimensional.

V. 0-dimensional spaces

In this part, we make strong assumptions on the separation properties of the space in question; in return we gain by assuming weaker homogeneity properties.

As an application we shall obtain a quite general resconstructibility result for 0-dimensional linear orderings (Theorem 3.50(b)).

We again drop all previous assumptions and start a new list of assumptions.

For $G \subseteq H(X)$ and $x \in X$ let $G(x)$ denote $\{g(x) \mid g \in G\}$.

Assumption 3.V.1. (a) $X$ is a Hausdorff space.

(b) For every $x \in X \quad|G(x)| \geq 3$.

(c) $R$ denotes a dense subset of $R(X)$.

Let $\varphi_{C}^{\prime}(V)$ be the following first-order formula in $L_{H R}$ : For every $f \in G$, if $f(V \cap f(V))=V \cap f(V)$ then there is $f^{\prime} \in G$ such that $f^{\prime} \supseteq f \uparrow(V \cap$ $f(V)) \cup \operatorname{Id} \uparrow \sim(V \cap f(V))$.

Let $\varphi_{C}(V) \equiv \varphi_{C}^{\prime}(V) \wedge \varphi_{C}^{\prime}(\sim V)$.

Definition 3.44. $V$ is strongly recognizably clopen if it is reconizably clopen (see Definition 3.12) and for every $g \in G$ if $g(V \cap g(V))=V \cap g(V)$, then $g \uparrow(V \cap g(V)) \cup \operatorname{Id} \uparrow(X-(V \cap g(V))) \in G$.

Note that in $M(X, H(X))$ every clopen set is strongly recognizably clopen.

Assumption 3.V.2. (a) Every clopen subset of $X$ is strongly recognizably clopen.

(b) For every distinct $x, y \in X$ there is a clopen $V \in R$ such that $x \in V$ and $y \notin V$.

Lemma 3.45. For every $V \in R: H R(X, G, R) \vDash \varphi_{C}[V]$ iff $V$ is clopen.

Proof. It is clear that $\varphi_{C}$ is satisfied by every clopen set.

To prove the converse we need the following claims.

Claim 1. If $U$ satisfies $\varphi_{C}^{\prime}, x \in \operatorname{bd}(U), g \in G$, and $g(x) \neq x$, then there is $W^{\prime}=W^{\prime}(U, x, g) \ni x$ such that $W^{\prime}$ is clopen and $g\left(W^{\prime} \cap U\right) \subseteq \sim U$. Proof. Let $W$ be a clopen neighborhood of $x$ such that $g(W) \cap W=\varnothing$. Let $h \in G$ extend $g \uparrow W \cup g^{-1} \uparrow g(W) \cup \mathrm{Id} \uparrow \sim(W \cup g(W))$. Since $h^{2}=$ Id $h(U \cap h(U))=U \cap h(U)$, hence since $U$ satisfies $\varphi_{C}^{\prime}$ there is $f \in G$ extending $h \uparrow(U \cap h(U)) \cup \operatorname{Id} \uparrow \sim(U \cap h(U))$. We show that $S \stackrel{\text { def }}{=} W \cap U \cap h(U)$ is clopen. $f(S)=h(S)=g(S) \subseteq g(W)$ and $f(W-S)=W-S \subseteq W$, and since $W$ and 
$g(W)$ are disjoint and clopen $\operatorname{cl}(S) \cap \operatorname{cl}(W-S)=\varnothing$, hence $S$ is clopen. Let $W^{\prime}=W-S$, then $W^{\prime}$ is as required. This proves Claim 1 .

Claim 2. Let $U$ satisfy $\varphi_{C}, x \in \operatorname{bd}(U), g \in G$, and $g(x) \neq x$; then there is $W=W(U, x, g) \ni x$ such that $W$ is clopen $g(W \cap U)=g(W) \sim U$ and $g(W \sim U)=g(W) \cap U$. Proof: Let $W=W^{\prime}(U, x, g) \cap W^{\prime}(\sim U, x, g)$; then $W$ is as required.

Let $H R(X, G, R) \vDash \varphi_{C}[V]$, and suppose by contradiction that $V$ is not clopen. Let $x \in \mathrm{bd}(V)$. By Assumption 3.V.1(b) there are $g_{1}, g_{2} \in G$ such that $x \neq g_{1}(x) \neq g_{2} g_{1}(x) \neq x$. Let $W_{1}=W\left(V, x, g_{1}\right), W_{2}=W\left(V, g_{1}(x), g_{2}\right)$, and $W=g_{1}^{-1}\left(W_{2}\right) \cap W_{1}$.

$$
\begin{aligned}
g_{2} g_{1}(W \cap V) & =g_{2} g_{1}\left(W_{1} \cap g_{1}\left(W_{2}\right) \cap V\right)=g_{2}\left(g_{1}\left(W_{1} \cap V\right) \cap W_{2}\right) \\
& =g_{2}\left(\left(g_{1}\left(W_{1}\right) \sim V\right) \cap W_{2}\right) \subseteq g_{2}\left(W_{2} \sim V\right) \subseteq V .
\end{aligned}
$$

Hence $g_{2} g_{1}(W \cap V) \subseteq V$. This contradicts the existence of $W\left(V, x, g_{2} g_{1}\right)$, hence $V$ is clopen, and the lemma is proved.

Lemma 3.46. (a) Let $\varphi_{P}(V)$ be the formula in $L_{H R}$ which says: for every clopen $U$ either $V \cap U$ is clopen or $V \sim U$ is clopen, but not both of them are clopen. Then for every $V \in R \quad H R(X, G, R) \vDash \varphi_{P}[V]$ iff $|\operatorname{bd}(V)|=1$.

(b) Let $\varphi_{\mathrm{Eq}}\left(U_{1}, U_{2}\right)$ be the formula which says: for every clopen $V: U_{1} \cap V$ is clopen iff $U_{2} \cap V$ is clopen.

Then for every $U_{1}, U_{2} \in R$ : if $\left|\operatorname{bd}\left(U_{1}\right)\right|=\left|\operatorname{bd}\left(U_{2}\right)\right|=1$, then $H R(X, G, R)$ $\vDash \varphi_{\mathrm{Eq}}\left[U_{1}, U_{2}\right]$ iff $\mathrm{bd}\left(U_{1}\right)=\operatorname{bd}\left(U_{2}\right)$.

Proof. Easy.

Assumption 3.V.3. $R$ contains a base for $X$ consisting of clopen sets.

Lemma 3.47. Let $\varphi_{\in}(U, V)$ be the formula in $L_{H R}$ which says that there is a clopen $W \subseteq V$ such that $U \cap W$ is not clopen.

Then for every $U, V \in R$ if $|\operatorname{bd}(U)|=1$, then $H R(X, G, R) \vDash \varphi_{\in}[U, V]$ iff $\operatorname{bd}(U) \subseteq V$.

Proof. Easy.

Let $K^{T D}$ be the class of all $M(X, G)$ 's which have the following properties: (1) $X$ is a Hausdorff space; (2) the set of clopen sets is an open base for $X$; (3) for every $x \in X$ either $\{x\}$ is open or there is a regular open set $V$ such that $\operatorname{bd}(V)=\{x\} ;$ (4) for every $x \in X|G(x)| \geq 3$; (5) for every open $T$ : if $|T|>1$, then there is $g \in G-\{\operatorname{Id}\}$ such that $\operatorname{var}(g) \subseteq T$; and (6) every clopen set is strongly recognizably clopen.

Definition 3.48. $X$ is a linear space if its topology is the order topology of a dense linear ordering without end points.

Proposition 3.49. (a) For first countable spaces (1) $\wedge$ (2) implies (3).

(b) If $X$ is linear and $M(X)$ satisfies (2), (4), and (5), then $M(X) \in K^{T D}$. Proof. Easy. 
Let $K^{T D O}$ be the set of all $M(X)$ 's in $K^{T D}$ such that $X$ is linear. So $M(X) \in K^{T D O}$ if (i) $X$ is linear; (ii) for every distinct $x, y \in X$ there is a clopen $V$ such that $x \in V$ and $y \notin V$; (iii) for every nonempty open $T$ there is $g \in H(X)-\{$ Id $\}$ such that $\operatorname{var}(g) \subseteq T$; and (iv) for every $x \in X$ $|H(X)(x)| \geq 3$.

Let $K_{0}^{T D}=\left\{G \mid \exists X\left(G \subseteq H(X) \wedge M(X, G) \in K^{T D}\right)\right\}, K_{3}^{T D}=\{H R P(X, G) \mid$ $\left.M(X, G) \in K^{T D}\right\}, K_{0}^{T D O}=\left\{H(X) \mid M(X) \in K^{T D O}\right\}$, and

$$
K_{3}^{T D O}=\left\{H R P\left((X, H(X)) \mid M(X) \in K^{T D O}\right\} .\right.
$$

Theorem 3.50. (a) $K_{3}^{T D}$ is interpretable in $K_{0}^{T D}$; hence if $M\left(X_{i}, G_{i}\right) \in K^{T D}$, $i=1,2$, and $G_{1} \cong G_{2}$, then $X_{1} \cong X_{2}$.

(b) $K_{3}^{T D O}$ is interpretable in $K_{0}^{T D O}$.

Proof. (a) One would like to use 2.15 in order to interpret $K_{1}^{T D} \stackrel{\text { def }}{=}\{H V(X, G) \mid$ $\left.M(X, G) \in K^{T D}\right\}$ in $K_{0}^{T D}$. However, in 2.15 it is assumed that $M(X, G)$ is regionally disrigid, whereas here $X$ might have isolated points.

Let $I S(X)=\{x \mid\{x\}$ is clopen in $X\}$; let $V^{I S}(X)=\sum\{\{x\} \mid x \in I S(X)\}$. Note that by requirement (5) in the definition of $K^{T D}$, every transposition of isolated points belongs to $G$. So the methods of [R1] can be used in order to interpret $I S(X)$ in $G$. Let $\varphi: P(I S(X)) \rightarrow\left\{V \mid V \in R(X)\right.$ and $\left.V \subseteq V^{I S}(X)\right\}$ be defined as follows: $\varphi(A)=\operatorname{int}(\operatorname{cl}(A))$. Clearly $\varphi$ is an isomorphism between the above Boolean algebras. Since $I S(X)$ can be interpreted in $G, P(I S(X))$ can be interpreted in $G$, and hence $\left\{V \in R(X) \mid V \subseteq V^{I S}(X)\right\}$ is interpretable in $G$. The method of 2.15 can be applied in order to interpret in $G\{V \in$ $\left.R(X) \mid V \subseteq X \sim V^{I S}(X)\right\}$.

It is possible to combine the interpretations of $\left\{V \in R(X) \mid V \subseteq V^{I S}(X)\right\}$ and $\left\{V \in R(X) \mid V \subseteq X \sim V^{I S}(X)\right\}$ and to obtain in this way an interpretation of $R(X)$ in $G$; moreover in this way one gets an interpretation of $H R(X, G)$ in $G$. By Lemmas 3.46 and 3.47 it follows that $\operatorname{HRP}(X, G)$ is interpretable in $H R(X, G)$. So $H R P(X, G)$ is interpretable in $G$.

Suppose now that for $i=1,2 M\left(X_{i}, G_{i}\right) \in K^{T D}$ and that $G_{1} \cong G_{2}$. So $H R P\left(X_{1}, G_{1}\right) \cong H R P\left(X_{2}, G_{2}\right)$; by requirement (2) in the definition of $K^{T D}$ $R\left(X_{i}\right)$ are open bases for $X_{i}$, so $X_{1} \cong X_{2}$. We have thus proved (a).

(b) is a special case of (a).

Question 6. Can (b) be strengthened by replacing "interpretation" by "first-order interpretation"? The only difficulty is to show that $V(X, H(X))=R(X)$.

Remark. (a) If in the definition of $K^{T D O}$ we strengthen the requirement of regional disrigidity by demanding that for every nonempty open set $T$ there is an order-preserving $g \in H(X)-\{$ Id $\}$ such that $\operatorname{var}(g) \subseteq T$, then for the resulting class we can obtain a first-order interpretation of $H R P(X, H(X))$ in $H(X)$. 
(b) It is impossible to drop the requirement of dense linear orderings without end points. The following linear orderings have the same group of homeomorphisms: $\left(\aleph_{1}+1\right)^{*}+\aleph_{1}+1$ and $\aleph_{1}^{*}+\aleph_{1}$.

VI. Some more faithful classes.

Let $V$ be a topological vector space over an ordered field $F$; a subset $U \subseteq V$ is linearly bounded if the intersection of $U$ with every straight line $l$ is bounded in $l$. Let $\mathscr{V} \stackrel{\text { def }}{=}\{V \mid V$ is locally convex topological vector space over an ordered field $F$, and $V$ contains a nonempty linearly bounded open subset $U$, and if $\mathbf{R}$ is not embeddable in $F$ then $U$ is clopen $\}$. We will show that the definition of $K^{M}$ (following 3.34) can be modified to include $K^{T D}$ of $3 . \mathrm{V}$ and the class $K^{L L} \stackrel{\text { def }}{=}\{X \mid X$ is a manifold over $\mathscr{V}\}$.

Let us redefine $S(X, G)$ (previously defined after 3.9). $S(X, G)=\{x \mid$ $x$ belongs to an excellently structured small component of $M(X, G)\} \cup\{x \mid$ for some clopen $U \quad x \in U$ and $\left.M(U,\{g \in G \mid \operatorname{var}(g) \subseteq U\}) \in K^{T D}\right\}$. Let $K^{E C S}=\left\{M(X, G) \mid M(X, G) \in K^{*}\right.$, every clopen set in $X$ is strongly recognizably clopen, and every small component that does not contain a small clopen set is excellently structured \}; see $3.13(\mathrm{c}) . K^{M}$ is defined from $K^{E C S}$ as in 3.III following 3.34.

Theorem 3.51. (a) $K^{T D} \subseteq K^{M}$.

(b) $K^{L L} \subseteq K^{M}$.

(c) $K^{M}$ is interpretable in $\left\{G \mid \exists X\left(M(X, G) \in K^{M}\right)\right\}$.

Proof. (a) is clear from the definition; the proof of (c) is included in 3.II, 3.III, and 3.V.

(b) It suffices to show that if $X \in K^{L L}$ is a topological vector space over $F$, then (1) if $F \cong \mathbf{R}$, then $X$ is excellently structured, and (2) if $F \not \mathbf{R}$ then $X \in K^{T D}$.

(b1) Let $X \in K^{L L}$ be a topological vector space over $\mathbf{R}$. Let $U$ and $V$ be open linearly bounded convex open sets. We show that for some linearly bounded $W$ and $h \in H(X): h(U)=V$ and $\operatorname{var}(h) \subseteq W$. W.1.o.g. $0 \in U \subseteq V$. Let $\|x\|_{U}=\operatorname{Sup}(\{\lambda \mid x \notin \lambda U\})$ and $\|x\|_{V}$ be defined analogously. It is well known that \|\|$_{U},\|\|_{V}$ are continuous functions from $X$ to $\mathbf{R}$. Let

$$
h(x)= \begin{cases}0, & x=0, \\ \|x\|_{U} /\|x\|_{V} \cdot x, & x \neq 0 .\end{cases}
$$

Then $h \in H(X)$ and $h(U)=V . h$ can be corrected to be the identity outside $2 \mathrm{~V}$.

We have thus shown that $X$ is a small component of itself. By inessential modifications of 3.IV we can show that every linearly bounded convex nonempty open set $U$ is excellent and that $X$ is excellently structured.

(b2) Let $X \in K^{L L}$ be a locally convex topological vector space over $F$ and $F \not \mathbf{R}$ and suppose $X$ contains a linearly bounded nonempty open set $V_{0}$, 
which in the case that $F$ is Archimedean is also clopen. We show that there is a regular open set $U$ such that $\operatorname{bd}(U)=\{0\}$. This will imply that $X \in K^{T D}$.

First let us show that there is a sequence $\left\{U_{\beta} \mid \beta<\alpha\right\}$ of clopen sets such that for every limit $\delta<\alpha \bigcap_{\beta<\delta} U_{\beta}=U_{\delta}$ and $\bigcap_{\beta<\alpha} U_{\beta}=\{0\}$.

Suppose that $F$ is Archimedean. W.1.o.g. $V_{0} \ni 0$; let $U_{i}=(1 / i) \cdot V_{0}$ where $i<\omega$. It is clear that $\left\{U_{i} \mid i<\omega\right\}$ is as required. Suppose $F$ is not Archimedean. Let $V_{1}$ be an open convex symmetric linearly bounded neighborhood of 0 . Let $V=\bigcup_{n \in \omega} n V_{1}$. We first show that $V$ is clopen. Suppose $x \notin V$, then $(x+V) \cap V=\varnothing$ for otherwise there are $v_{1}, v_{2} \in V$ $x+v_{1}=v_{2}$, hence $x=\frac{1}{2}\left(2 v_{2}\right)+\frac{1}{2}\left(-2 v_{1}\right) \in V$. Let $\left\{\lambda_{i} \mid i<\alpha\right\}$ be a strictly decreasing sequence of members of $F$ converging to 0 , and $\lambda_{0}<1$. For every $\beta \leq \alpha$ let $U_{\beta}=\bigcap_{i<\beta} \lambda_{i} V$. It is trivial that $U_{\alpha}=\{0\}$ and that for a limit $\delta$ $U_{\delta}=\bigcap_{\beta<\delta} U_{\beta}$. We prove that $U_{\beta}$ is open for every $\beta<\alpha$. Let $x \in U_{\beta}$; we show that $x+\lambda_{\beta} V \subseteq U_{\beta}$. Let $i<\beta$; hence $x=\lambda_{i} v$ for some $v \in V$, thus $x+\lambda_{\beta} V=\lambda_{i}\left(v+\left(\lambda_{\beta} / \lambda_{i}\right) \cdot V\right) \subseteq \lambda_{i}(V+V)=\lambda_{i} V$. Hence $x+\lambda_{\beta} V \subseteq U_{\beta}$.

Let $l$ be a ray with endpoint at 0 . The set $A \stackrel{\text { def }}{=}\left\{i \mid\left(U_{i}-U_{i+1}\right) \cap l \neq \varnothing\right\}$ is unbounded in $\alpha$. For $i \in A$ let $W_{i} \subseteq U_{i}-U_{i+1}$ be a convex clopen set such that $W_{i} \cap l \neq \varnothing$. Let $W=\bigcup_{i \in A} W_{i}$. Let us denote $X=U_{-1}$. Hence for every $i \quad U_{i}-U_{i+1}$ is clopen and $U_{i<\alpha}\left(U_{i}-U_{i+1}\right)=X-\{0\}$. It follows that $\operatorname{bd}(W)-\{0\}=\bigcup_{i<\alpha} \operatorname{bd}\left(W \cap\left(U_{i}-U_{i+1}\right)\right)$. But since $W \cap\left(U_{i}-U_{i+1}\right)$ is always clopen $\operatorname{bd}(W)-\{0\}=\varnothing$. Clearly $0 \in \operatorname{bd}(W)$, hence $\operatorname{bd}(W)=\{0\}$. It remains to show that $W$ is regular. $0 \notin \operatorname{int}(\operatorname{cl}(W))$ since $-l \cap \operatorname{cl}(W)=\{0\}$. Hence $\operatorname{int}(\operatorname{cl}(W))=W$. Q.E.D.

So far the classes for which we have shown faithfulness consisted of spaces $X$ which essentially had the following property: for every $x \in X$ either $x$ lies in a clopen connected component of $X$, or there is a clopen 0 -dimensional $U$ containing $x$. These requirements excluded spaces like $\mathbf{R} \times \mathbf{Q}, \mathbf{R} \times \mathbf{I}$, or $\mathbf{R} \times \mathbf{C}$, where $\mathbf{Q}, \mathbf{I}$, and $\mathbf{C}$ are respectively the rationals, the irrationals, and the Cantor set.

We can do a little bit better and include the above spaces as well as some other natural cases in faithful classes.

Let $C(X)$ denote the set of components of $X$ and let $G \subseteq H(X)$. For $x \in X, C \in C(X), U \in R(X)$ and $f, g \in G$ let $C(x)$ denote the connected component of $x, G(x)=\{g(x) \mid g \in G\}, \operatorname{Bd}(U, C) \stackrel{\text { def }}{\equiv}(C=\operatorname{bd}(U))$, and $\operatorname{Eq}(f, g, C) \stackrel{\text { def }}{\equiv}(f \uparrow C=g \uparrow C)$. Let $H R C(X, G)=\langle G, R(X), C(X) ; \mathrm{Op}$, $\subseteq, \mathrm{Bd}, \mathrm{Eq}\rangle$ where $\mathrm{Op}=\{\langle g, x, y\rangle \mid g \in G, x, y \in R(X) \cup C(X)$, and $g(x)=y\}$, and $\subseteq$ denotes the inclusion relation on $R(X)$.

We first show that under appropriate assumptions on $M(X, G), H R C(X, G)$ is interpretable in $G$. Let $G^{C F}=\{g \in G \mid(\forall C \in C(X))(g(C)=C)\}$ and $G^{L C F}=\{g \in G \mid(\forall C \in C(X))(|C|>1 \Rightarrow g(C)=C)\}$.

Let $K^{C}$ be the class of all $M(X, G)$ 's which satisfy the following requirements: (1) $X / C(X)$ is 0 -dimensional; (2) $(\forall C \in C(X))((C$ is not clopen $) \Rightarrow$ 
$(\exists V \in R(X))(C=\operatorname{bd}(V))) ;(3) M\left(X, G^{L C F}\right) \in K^{*} ;$ (4) for every $x \in X$ $G^{C F}(x)$ is dense in $C(x) ;(5)(\forall x \in X)(|G(x)| \geq 3)$; and (6) for every $g \in G$ and $U \in R(X)$ : if $g(U)=U$ and $g \nmid \operatorname{bd}(U)=\operatorname{Id}$, then $g \backslash U \cup \operatorname{Id} \uparrow(X-U) \in$ $G$.

Let $K_{0}^{C}=\left\{G \mid \exists X\left(M(X, G) \in K^{C}\right)\right\}$ and $K_{1}^{C}=\{H R C(X, G) \mid M(X, G) \in$ $\left.K^{C}\right\}$.

Lemma 3.52. (a) $K_{1}^{C}$ is interpretable in $K_{0}^{C}$.

(b) If $X=Y \times Z, Y \in K^{E C S}$, and $Z \in K^{T D}$, then $M(X) \in K^{C}$.

(c) If $X=\prod_{i \in \omega} X_{i}$, and for every $i X_{i}$ is a Euclidean manifold, then $M(X) \in K^{C}$.

(d) If $X$ is a box product of Euclidean manifolds then $M(X) \in K^{C}$.

Proof. We leave the easy proofs of (b), (c), and (d) to the reader and prove only (a).

Since $G^{L C F}$ is a normal subgroup of $G$, it follows from 2.14 and requirement (3) in the definition of $K^{C}$ that $H R(X, G)$ is interpretable in $G$.

We will use the formulas $\varphi_{C}, \varphi_{P}, \varphi_{\mathrm{Eq}}$, and $\varphi_{\in}$ of 3.V. Let $\varphi_{P}^{\prime}(V)=$ $\varphi_{P}(V) \wedge(V$ is the sum of clopen sets). It is easy to modify the proof of $3 . V$ in order to show that $\varphi_{C}(U), \varphi_{P}^{\prime}(V), \varphi_{\mathrm{Eq}}\left(U_{1}, U_{2}\right)$, and $\varphi_{\epsilon}(U, V)$ mean respectively that $U$ is clopen, $\operatorname{bd}(V) \in C(X), \operatorname{bd}\left(U_{1}\right)=\operatorname{bd}\left(U_{2}\right)$, and $\operatorname{bd}(U) \subseteq V$. It follows that $\langle G, R(X), C(X) ; \mathrm{Op}, \mathrm{Bd}\rangle$ is interpretable in $G$.

Let $\mathrm{Eq}_{0}(g, C) \equiv \exists V(C=\mathrm{bd}(V) \wedge(g \nmid V \cup \mathrm{Id} \backslash(X \sim V) \in G))$. It is easy to see that $\mathrm{Eq}_{0}(g, C)$ holds iff $g \mid C=\mathrm{Id}$, so $\operatorname{Eq}(g, f, C)$ can be expressed by the formula $\mathrm{Eq}_{0}\left(g f^{-1}, C\right)$. This proves the lemma.

Recall that $K^{L C}=\{M(X, G) \mid X$ is locally compact and has no isolated points, and for every $x \in X$ and an open $T \ni x\{g(x) \mid g \in G$ and $g \mid$ $(X-T)=\mathrm{Id}\}$ is somewhere dense $\}$. For $A \subseteq X$ let $G \mid A=\{g|A| g \in G$ and $g(A)=A\}$, hence $G \mid A \subseteq H(A)$. Let $K^{C L C}=\{M(X, G) \mid M(X, G) \in$ $K^{C}$ and for every $C \in C(X)$ if $|C|>1$ then $\left.M(C, G \mid C) \in K^{L C}\right\}$ and $K_{0}^{C L C}=\left\{G \mid \exists X\left(M(X, G) \in K^{C L C}\right)\right\}$.

Analogously we define $K^{C E C S}$ to be the class of all $M(X, G)$ 's in $K^{C}$ such that for every $C \in C(X)$ if $|C|>1$ then $M(C, G \mid C) \in K^{E C S}$, and we define $K_{0}^{C E C S}$ accordingly.

Theorem 3.53. (a) $K^{C L C}$ is interpretable in $K_{0}^{C L C}$.

(b) If $X=\prod_{i \in \omega} X_{i}$, for every $i \quad X_{i}$ is a Euclidean manifold, $X_{i}$ is compact for all but finitely many $i$ 's, and $X$ does not have isolated points, then $M(X) \in$ $K^{C L C}$. (Here a space consisting of a single point is considered to be a manifold.)

(c) $K^{C E C S}$ is interpretable in $K_{0}^{C E C S}$.

(d) If $X \in K^{E C S}$ and $Y \in K^{T D}$, then $M(X \times Y) \in K^{C E C S}$, in particular $\mathbf{R} \times \mathbf{Q}, \mathbf{R} \times \mathbf{C}, \mathbf{R} \times \mathbf{I} \in K^{C E C S}$. 
Proof. We leave the easy proofs of (b) and (d) to the reader; the proofs of (a) and (c) are completely analogous, so we prove only (a). It suffices to show that $M(X, G)$ is interpretable in $H R C(X, G)$. Let $M^{\prime}(X, G)=\langle G, R(X), X ;$ Op , $\subseteq\rangle . M^{\prime}(X, G)$ differs from $M(X, G)$ since the $\in$ relation on $X \times R(X)$ does not appear in $M^{\prime}(X, G)$. However, $M(X, G)$ is interpretable in $M^{\prime}(X, G)$ because $x \in U$ is equivalent to the formula $\exists g(g(x) \neq x \wedge g \mid \sim U=I d)$. Hence it suffices to show that $M^{\prime}(X, G)$ is interpretable in $\operatorname{HRC}(X, G)$. Let $C_{1}(X)=\{x \mid\{x\}=C(x)\}$ and $C_{2}(X)=X-C_{1}(X)$. It is trivial that $\left\langle G, R(X), C_{1}(X) ; \mathrm{Op}, \subseteq\right\rangle$ is interpretable in $\operatorname{HRC}(X, G)$. To interpret $C_{2}(X)$ note that for every $C \in C(X)$

$$
G \mid C \cong\{g \in G \mid g(C)=C\} /\{g \in G|g| C=\mathrm{Id}\} .
$$

The right-hand group is interpretable in $\operatorname{HRC}(X, G)$, and so is the left-hand group. By 3.I we thus obtain that $C_{2}(X)$ can be captured from $H R C(X, G)$. This concludes the proof of (a).

\section{BIBLIOGRAPHY}

[BP] C. Bessaga and A. Pelczynski, Selected topics in infinite dimensional topology, Polish Scientific Publishers, Warsaw, 1975.

[CK] C. C. Chang and H. J. Keisler, Model theory, North-Holland, Amsterdam, 1973.

[En] S. J. Eigen, The group of measure preserving transformations of $[0,1]$ has no outer automorphisms, Math. Ann. 259 (1982), 259-270.

[Fl] R. P. Filipkiewicz, Isomorphism between diffeomorphism groups, Ergodic Theory Dynamical Systems 2 (1982), 159-171.

[GGHJ] A. M. W. Glass, Y. Gurevich, W. C. Holland, and M. Jambu-Giraudet, Elementary theory of automorphism groups of doubly homogeneous chains, preprint.

[Gm] V. K. A. M. Gugenheim, Piecewise linear isotopy, Proc. London Math. Soc. 31 (1953), 29-53.

[L1] Yu-lee Lee, Characterizing the topology by the class of homeomorphism, Duke Math. J. 35 (1968), 625-629.

[L2] __ On the existence of non-comparable homogeneous topologies with the same class of homeomorphisms, Tôhoku Math. J. (2) 22 (1970), 499-501.

[Lg1] W. Ling, A classification theorem for manifold automorphism groups, preprint.

[Lg2] __ Factorizable groups of homeomorphisms, preprint.

[M] R. A. McCoy, Homeomorphism groups of Hilbert cube manifolds, General Topology Appl. 2 (1972), 55-60.

[Ml] J. van Mill, Homeomorphism groups and homogeneous spaces, preprint.

[Mm] D. Maharam, On homogeneous measure algebras, Proc. Nat. Acad. Sci. U.S.A. 28 (1942), 108-111.

[R1] M. Rubin, On the automorphism groups of homogeneous and saturated Boolean algebras, Algebra Universalis 9 (1979), 54-86.

[R2] _ On the reconstruction of complete Boolean algebras from their automorphism groups, Arch. Math. Logik und Grundlagen. 20 (1980), 125-146.

[R3] _ Second countable connected manifolds with elementarily equivalent homeomorphism groups are homeomorphic in the constructible universe, in preparation.

[RY] M. Rubin and Y. Yomdin, On the reconstruction of smooth manifolds, Banach spaces and measure spaces from their automorphism groups, Israel $\mathbf{J}$. of Math. (to appear). 
[Rr] L. Rieger, Some remarks on automorphisms of Boolean algebras, Fund. Math. 38 (1951), 209216.

[S] S. Shelah, Why there are many nonisomorphic models for unsuperstable theories (Proc. Internat. Congr. Math., Vancouver, B. C., 1974, vol. 1), Canadian Math. Congress, Montreal, 1975, pp. 259-263.

[Sp] E. H. Spanier, Algebraic topology, McGraw-Hill, New York, 1966.

[Ts] F. Takens, Characterization of a differentiable structure by its group of diffeomorphisms, Bol. Soc. Brasil Mat. 10 (1979), 17-26.

[W] J. V. Whittaker, On isomorphic groups and homeomorphic spaces, Ann. of Math. 78 (1963), 74-91.

Department of Mathematics, Ben Gurion University, Beer Sheva, Israel (Current address)

Department of Mathematics, Simon Fraser University, Burnaby, Canada

E.Mail.matti@bengus.bitnet 